\title{
A MASH Zone Revealed: the Mafic Complex of the Sierra Valle Fértil
}

\author{
Barry A. Walker, Jr$^{1}$, George W. Bergantz ${ }^{1 *}$, Juan E. Otamendi ${ }^{2}$, \\ Mihai N. Ducea ${ }^{3}$ and Eber A. Cristofolini ${ }^{2}$
}

${ }^{1}$ Department of Earth and Space Sciences, University of Washington, Box 351310, Seattle, WA 98195, USA; ${ }^{2}$ Departamento de Geología, Universidad Nacional de Río Cuarto, X5804BYA, Río Cuarto, Argentina and ${ }^{3}$ Department of Geosciences, University of Arizona, Tucson, AZ 85721, USA

*Corresponding author. E-mail: bergantz@u.washington.edu

Received March 5, 2015; Accepted September 14, 2015

\begin{abstract}
The Sierra Valle Fértil Complex of west-central Argentina represents a section of the Ordovician ( $\sim 70 \mathrm{Ma}$ ) Famatinian arc and exposes a continuous, tilted crustal arc section ranging in depth from $\sim 12$ to $32 \mathrm{~km}$ ( 4-8 kbar pressure). This arc section exposes the complete compositional architecture from ultramafic and mafic rocks to upper crustal granodiorites. Field and compositional data are presented to document the deep ( 6-8 kbar) mafic complex of the Sierra Valle Fértil. The mafic complex is composed of many tens to hundreds of plutonic cumulate bodies in a complex and non-regular arrangement. There is no simple compositional, kinematic or age relationship between neighboring plutons throughout the section, as expressed by cumulate compositions, emplacement horizon, size, composition, texture or style of contact. Amphibole gabbronorites and mafic tonalites dominate, but norites, amphibole websterites, troctolites and minor anorthosites are present. Amphibole is common but always as a replacement phase, and is never observed undergoing subsequent dehydration melting. Hence there is no evidence that voluminous tonalites were produced by dehydration melting of mafic precursors. A field-based, cumulateremoval fractionation model is presented that produces the observed compositional variations in five steps. Isotopic compositions of $\mathrm{Sr}$ and $\mathrm{Nd}$ deviate significantly from primitive mantle values, indicating a crustal contribution; however, this hybridization appears to have played a minor role in the major element evolution of the mafic complex. We interpret this isotopic and elemental decoupling as a byproduct of prolonged, punctuated MASH (melting, assimilation, storage, homogenization) processes in the lower crust. Isotopes may be the only residual evidence of assimilation within the mafic zone. This requires that melt removal from the cumulates was extraordinarily efficient.
\end{abstract}

Key words: igneous petrology; crustal contamination; island arc; MASH zone; magma chamber

\section{INTRODUCTION}

There is agreement that most arc magmatism is fundamentally basaltic (Hildreth, 1981); however, the depths and specific processes whereby basalt gives rise to intermediate and silicic magmas remain in question. The dominant paradigm describing this process remains the MASH (melting, assimilation, storage, homogenization) hypothesis of Hildreth \& Moorbath (1988). In this model, base-level geochemical attributes of arc magmas are established as mantle-derived basalts mix with basaltic precursors including cumulates, melt the lower crustal rocks and reside in an environment of continuing hybridization and melt removal. Numerical simulations of silicic melt generation by direct crustal melting (Bergantz, 1992; Dufek \& Bergantz, 2005; Annen et al., 2006) have been equivocal on the efficiency of this process, in part because they assume that assimilation is driven by simple contact melting, which is not energetically efficient (Barboza \& Bergantz, 1996, 2000). Hence the possibility of intermediate magma 
production occurring in lower crustal zones of anatexis rather than MASH zones, where pre-existing rock-as opposed to mantle-derived basalt-is the dominant mass contributor (Haraguchi et al., 2003; Saito et al., 2004, 2007; Ratajeski et al., 2005; Sisson et al., 2005; Clemens et al., 2011; Coleman et al., 2012) is controversial. Field-testing of these models has proven difficult. Numerous tilted batholiths provide cross-sectional views (Dilles, 1987; Tepper et al., 1993; Bachl et al., 2001; Miller \& Miller, 2002; Westerman et al., 2004; Kamiyama et al., 2007; Walker et al., 2007; Economos et al., 2008; Needy et al., 2009; Longo et al., 2010; Putirka et al., 2014; Ducea et al., 2015), but most of these batholiths expose only the uppermost several kilometers of the magma system and are dominated by intermediate to evolved compositions.

The structure of deep arc crust is thus constrained by very few examples. In the absence of exposure, cognate inclusions in volcanic rocks (Conrad \& Kay, 1984; Ducea \& Saleeby, 1998; Lee et al., 2001; Rodriguez-Vargas et al., 2005; Smith, 2014; Ducea et al., 2015) and seismic profiles across active arcs (Holbrook et al., 1999; Yuan et al., 2002; Kitamura et al., 2003; Calvert et al., 2008) are common proxies. Deeper exposures are often isolated blocks, beheaded and translated by faults that obscure their original position and regional context (Ross, 1985; Vernon et al., 1989; DeBari, 1994; Ducea et al., 2003; Kidder et al., 2003; Dessimoz et al., 2012; Putirka et al., 2014). However, there are a few tilted sections that provide relatively continuous, mid-deep arc crust exposures on the scale of tens of kilometers: the Kohistan batholith, Pakistan (Burg et al., 1998; Jagoutz et al., 2007; Jagoutz, 2010); the North Cascades, USA (Miller \& Paterson, 2001; Miller et al., 2009); the Talkeetna arc, Alaska (DeBari \& Coleman, 1989; Greene et al., 2006; Rioux et al., 2007; Hacker et al., 2008); the Bonanza arc, Canada (Cui \& Russell, 1995; DeBari et al., 1999; Larocque \& Canil, 2010); the Wooley Creek batholith, California (Coint et al., 2013) and the Sierra Valle Fértil complex, Argentina (Otamendi et al., 2009a; Ducea et al., 2010; Tibaldi et al., 2013). These field examples provide the basis for our current understanding of arc cross-sections.

In this contribution we focus primarily on the mafic complex of the Ordovician Sierra Valle Fertil (SVF) complex in northwestern Argentina, which exposes compositionally stratified, igneous arc crust interlayered with metasedimentary host-rocks at paleodepths of 12-32 km (Otamendi et al., 2009a; Tibaldi et al., 2013). We document the field relations, scales of compositional diversity and age relationships for a deep section within the SVF mafic zone. From our findings, several inferences arise. Although magmatism has almost completely reconstituted the crust in this arc section, the mafic zone is characterized by heterogeneity at the centimeter to kilometer scale. Gabbronorites are interlayered with tonalites in a sheet-like, but dominantly gradational and erratic fashion. Large, coherent batholiths are not present in the mafic complex. However, this heterogeneity ends rather abruptly in the transition zone to a relatively homogeneous section of more typical arc tonalites and granodiorite batholiths that form the upper part of the section. Many of the mafic rocks do not approximate melt compositions and are interpreted to be cumulates from which evolved melt was expelled. The original textures have been variably re-equilibrated, making identification of melt-present processes difficult. Despite this, there is abundant field evidence to support a model of fractional crystallization and melt transfer combined with partial melting of the metasedimentary host-rocks for the petrogenesis of the SVF mafic complex.

\section{GEOLOGICAL SETTING}

\section{The Famatinian Arc}

The Sierra Valle Ferril (SVF) is part of the midOrdovician to Devonian Famatinian subduction complex that is exposed along an $\sim 2500 \mathrm{~km}$ NW-SE swath in western Bolivia and Argentina (Fig. 1). In the north $\left(\sim 23^{\circ} \mathrm{S}\right)$, Famatinian arc exposures include calc-alkaline volcanic rocks, volcaniclastic rocks, sparse gabbroic mafic roots and high-level S-type plutonic rocks. The deepest Famatina exposures are between $\sim 28^{\circ}$ and $33^{\circ} \mathrm{S}$ where the Nazca plate is currently subducting at a relatively low angle (Ducea et al., 2010). The Famatinian arc represents some of the earliest subduction magmatism recognized in western South America and is interpreted to be the nascent stages of the Andean subduction system (Acenolaza et al., 2002). Subduction magmatism began in the region around 550 Ma during the Pampean Orogeny (Rapela et al., 1998). There was a hiatus in magmatism from $\sim 520$ to $495 \mathrm{Ma}$. During this time unroofing of the Pampean orogen and subsequent sedimentation (Cristofolini et al., 2012) created a thick $(>2000 \mathrm{~m})$ and widely distributed clastic sedimentary basin fill (regionally called the Puncoviscana, Suncho, Aguaditas, and Negro Peniado). Subsequently, magmatism stepped to the west. It was on and within the thick Puncoviscana sedimentary basin at the western edge of the proto-South American continent that the Famatinian arc was constructed. The arc was established at $\sim 495 \mathrm{Ma}$ (Pankhurst et al., 1998) and was active until $\sim 440 \mathrm{Ma}$ (Collo et al., 2009). In the specific vicinity of the Sierra Valle Fértil, laser ablation multicollector inductively coupled plasma mass spectrometry (LA-MC-ICP-MS) data for zircon indicate an age range of 485-465 Ma. Uranium-Pb data for zircons (chemical abrasion thermal ionization mass spectrometry; CATIMS), however, suggest that the initiation, operation, and death of this arc proceeded relatively rapidly-over an interval of less than $10 \mathrm{Myr}$ and perhaps as little as 5 Myr from 472 to $467 \mathrm{Ma}$ (Casquet et al., 2012). Arc cessation in the south by $\sim 465 \mathrm{Ma}$ is generally attributed to a collision with the Cuyania microplate (Thomas \& Astini, 1996; Cristofolini et al., 2014), but the reader is referred to the papers by Mulcahy et al. $(2007,2014)$ for further discussion. 


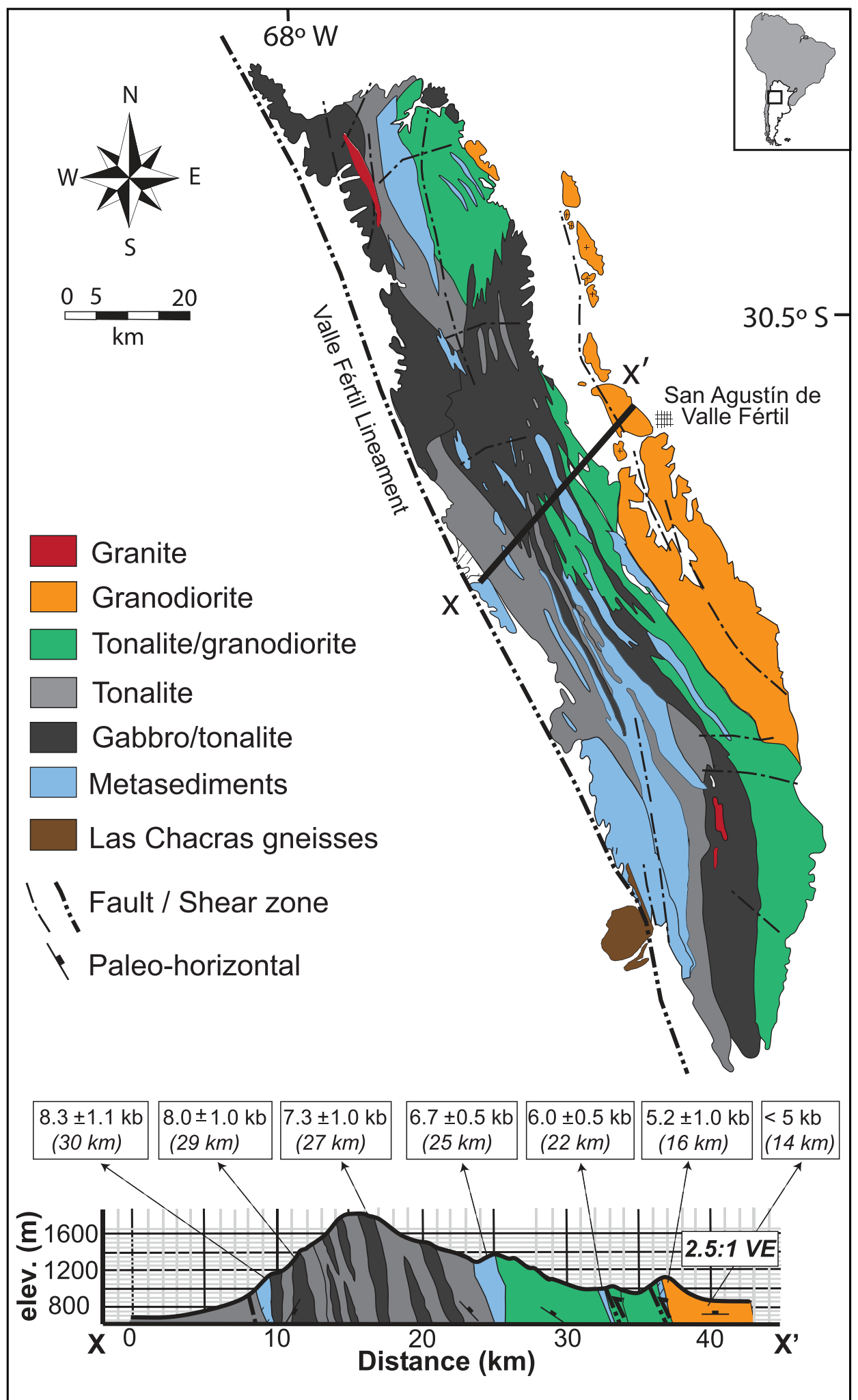

Fig. 1. Geological map of the Sierra Valle Fértil modified from the maps of Mirré (1976), Vujovich et al. (1996), Otamendi et al. $(2009 \mathrm{~b})$ and Tibaldi et al. (2013). Cross-section and barometery modified from Tibaldi et al. (2013).

\section{The Sierra Valle Fértil}

The Sierra Valle Fértil is located within the southern Famatinian belt and exposes eastward-tilted arc crust from paleodepths of $12-32 \mathrm{~km}$ (Tibaldi et al., 2013).
The SVF magmas intruded a thick, miogeoclinal sedimentary package dominated by metapelites, with minor marbles and graywackes (Cristofolini et al., 2012, 2014). Otamendi et al. (2009a) have documented the vertical 
distribution of distinct lithological units. From shallow to deep, the exposures include nested tonalite-granodiorite plutons that show a transition to extremely heterogeneous tonalite, which then show a transition to dominantly gabbroic and tonalitic rocks. A regional shear zone-the Valle Ferrtil Lineament-truncates the base of the section (Cristofolini et al., 2014) (Fig. 1). Throughout the SVF screens and xenoliths of metasedimentary country rocks, ranging from centimeter to kilometer scale, are sparsely present as stromatic to diatexite migmatites. Peak metamorphism and melting of the country rocks is interpreted to have been synplutonic (Otamendi et al., 2008), and interaction between metasediment-derived leucogranite melts and the SVF magmas is observable in the field. Zircon age data (Ducea et al., 2010) suggest that the SVF represents a relatively brief episode towards the end of southern Famatinian magmatism ( 485-465 Ma); age data from our study area suggest even shorter timescales ( $<6 \mathrm{Myr}$; unpublished data). This study focuses on the mafic zone of the SVF-the deeper portion of the section dominated by gabbronorite, norite, tonalite and small ultramafic bodies.

\section{METHODS}

Samples representing the compositional range of the SVF mafic zone were crushed to pea gravel size and powdered in a tungsten carbide vessel using a shatterbox. Preference was given to fresh samples without alteration. Sample sizes were typically $\sim 0.5-1 \mathrm{~kg}$ of material, crushed and split. In addition to the data from previous SVF studies by Otamendi et al. (2008, 2009a, 2009b, 2010a, 2010b, 2012) and Ducea et al. (2010, 2014), 117 new whole-rock analyses are presented here.

Major and trace elements were analyzed by X-ray fluorescence (XRF, ThermoARL) at the GeoAnalytical lab at Washington State University as described by Johnson et al. (1999). Rare earth elements (REE) and selected trace elements were analyzed by ICP-MS (Agilent 7700, quadrupole mass spectrometer) at Washington State University. Whole-rock powders were analysed at the University of Arizona for $\mathrm{Sr}, \mathrm{Nd}$, and $\mathrm{Pb}$ isotopes by TIMS. Isotopic compositions were obtained using the techniques and standardization of Ducea \& Saleeby (1998) and Otamendi et al. (2012). For several samples thin sections were analyzed using the OEMSCAN technique at the Advanced Mineralogy Research Center at the Colorado School of Mines. OEMSCAN utilizes energy-dispersive $\mathrm{X}$-ray spectroscopy (EDS) to generate mineral maps of thin sections. These maps are translated into false color images from which mineral modes are calculated (Hoal et al., 2009). Mineral compositions for plagioclase, amphibole, clinopyroxene, orthopyroxene, and olivine were determined at the University of Washington using a JEOL 733 electron microprobe.

\section{LITHOLOGICAL UNITS OF THE MAFIC ZONE}

Here, we describe the rocks that form an $\sim 100 \mathrm{~km}^{2}$ area within the Valle Fértil mafic complex (Fig. 2). Exposure of fresh rock is found in several approximately eastwest-oriented canyons that strike roughly perpendicular to the paleo-up direction, providing several pseudo cross-sections of the mafic zone. Outside the canyons, exposed rock is weathered, and thick vegetation prevents accurate mapping and sampling. The mafic zone section is a complex, heterogeneous assemblage of tonalite-gabbronorite, punctuated by bodies (less than kilometer scale) of ultramafic rocks, metasediments, and granite. Tonalite dominates the shallower parts of the exposed area. Within the tonalite-dominated areas, discernible gabbronorite bodies are present, although they are generally not large $(\sim<1 \mathrm{~km})$ and cannot always be inferred to extend to neighboring canyons. The gabbronorites have variable pyroxene and amphibole abundances that classify their specific rock type (e.g. gabbronorite, norite, hornblende gabbronorite), although for simplicity here we refer to these rocks as 'gabbronorites' because this is the most common variant. Contacts between the tonalite and gabbronorite bodies are usually gradational on the meter scale. Foliation and internal contacts of the tonalite and gabbronorites are typically oriented NW-SE to NE-SW and steeply dipping, consistent with the dominant foliation and map distribution of the largest bodies within the Sierra Valle Fértil complex (see Fig. 1). In places, gabbro and tonalite exposures are separated by 'transitional' zones comprising fine-grained gabbronorite with small, diffuse, stringy domains of tonalite and coarse, crystalrich tonalite. These transitional zones have been observed to reach thicknesses of up to $30-40 \mathrm{~m}$ (in Usno and Turbante Canyons). Below, we discuss the tonalite and gabbronorite lithological units in more detail, as well as three other volumetrically minor units that are exposed throughout the section (see also Table 1): ultramafic cumulate rocks, fine-grained gabbroic dikes and small silicic intrusions. Highly foliated and migmatized metasedimentary host rock (cordierite/ garnet biotite gneiss) is also present locally, as are cordierite-bearing diatexite domains (see Otamendi et al., 2008; Tibaldi et al., 2013).

\section{Tonalite}

Tonalite exposures are heterogeneous on the decimeter to meter scale with respect to modes and structures, with wispy, blob-like, and sheet-like interlayering between modally variable packages being typical (Fig. 3). Mineral foliation, defined by elongated plagioclase, biotite and amphibole, is common. Mafic sheets less than $1 \mathrm{~m}$ thick, inclusions, and schlieren domains are common in the tonalite and occasionally make up $20-30 \%$ of the outcrop. These inclusions typically have contacts with the host that are gradational over $\sim 1 \mathrm{~cm}$. In places, mafic inclusions appear frozen in various states of reaction or digestion, resulting in a 'ghost-like' appearance 


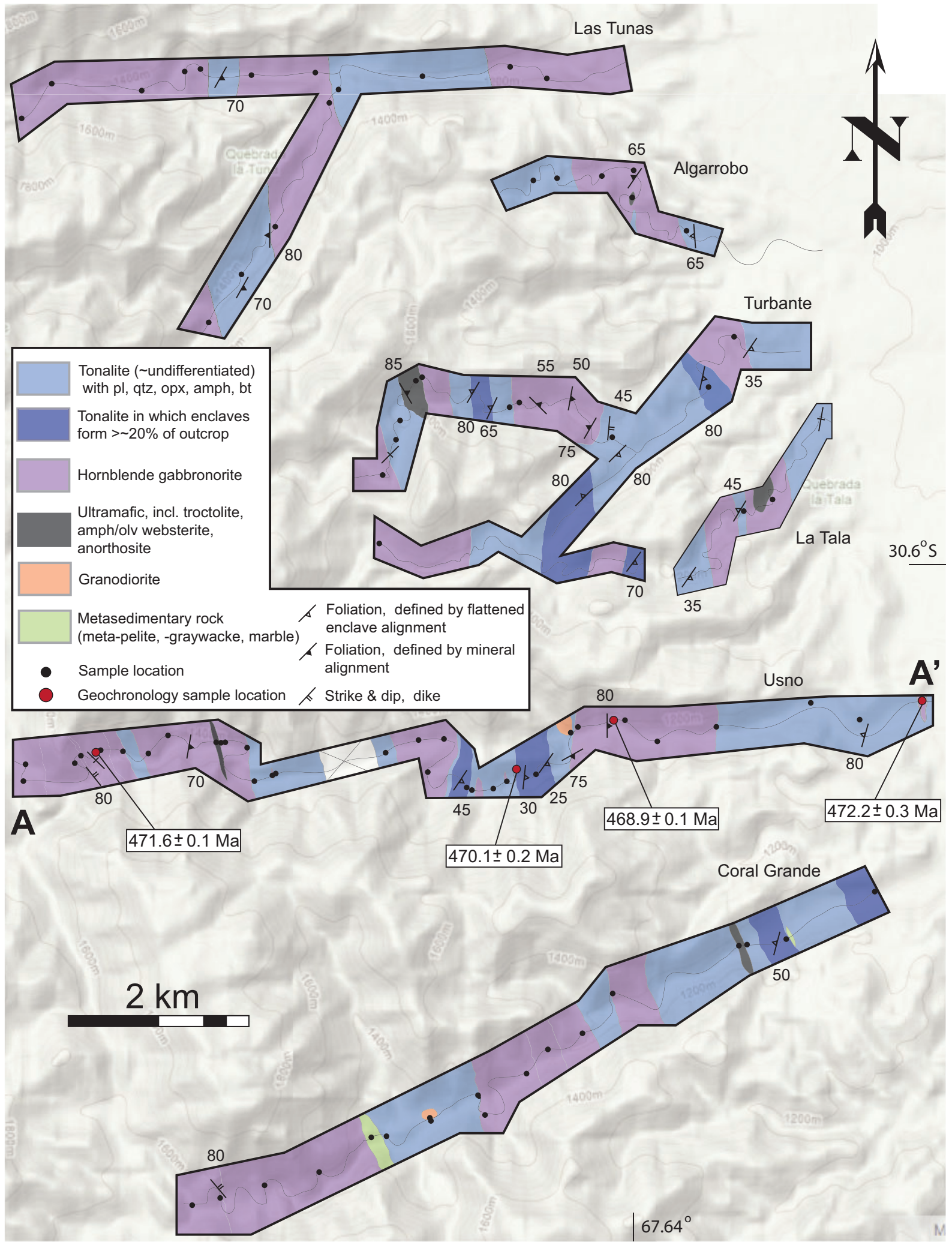

Fig. 2. Canyon-restricted geological map of a portion of the mafic zone of the Sierra Valle Fértil complex. Boundaries of units are approximate as contacts are often gradational over $\sim 1-40 \mathrm{~m}$. The orientation of unit boundaries is based on exposures in canyons. Also shown are sample locations and specifically indicated are those samples with CA-ID-TIMS zircon dates (unpublished data). (See text for details on lithological units.) 
Table 1: Petrographic descriptions of the main lithologies of the mafic zone

\begin{tabular}{|c|c|c|}
\hline Lithology & Petrographic characteristics & Modes \\
\hline Tonalite & $\begin{array}{l}\text { Medium to coarse grained. Very heterogeneous even at } \\
\text { thin-section scale. Inequigranular to equigranular. Plag } \\
\text { laths or domains are subhedral but commonly comprise } \\
\text { granophyric clusters. Qtz domains commonly have } \\
\text { consertal texture and exhibit subgrains. Amph generally } \\
\text { associated with opx as a rimming or reaction phase. Bio } \\
\text { and oxides also generally associated with opx }\end{array}$ & $\begin{array}{l}50-60 \% \text { plag }\left(\mathrm{An}_{74-83}\right) \\
10-50 \% \text { qtz } \\
0-15 \% \text { opx } \\
0-20 \% \text { amph ( } 5 \% \text { typical) } \\
0-15 \% \text { bio ( }<5 \% \text { typical }) \\
<5 \% \text { oxides } \\
\text { trace zircon, apatite }\end{array}$ \\
\hline Gabbronorite & $\begin{array}{l}\text { Fine to medium grained. Relatively equigranular. Plag } \\
\text { domains are subhedral and commonly granophyric. Cpx } \\
\text { and opx are granular and/or blocky and usually appear } \\
\text { clustered together. Amph usually either as a rimming or } \\
\text { reaction phase. In some rocks, amph is observed as } \\
\text { oikocrysts enclosing all other phases. Oxides are } \\
\text { interstitial }\end{array}$ & $\begin{array}{l}50-60 \% \text { plag }\left(\mathrm{An}_{82-96}\right) \\
<5 \% \text { qtz } \\
15-30 \% \text { opx } \\
5-20 \% \text { cpx } \\
1-6 \% \text { oxides } \\
5-30 \% \text { amph } \\
\text { trace zircon, apatite, } \\
\text { green spinel, pyrite }\end{array}$ \\
\hline Mafic dikes & $\begin{array}{l}\text { Fine grained. Plag and amph dominate and both minerals } \\
\text { are observed as subhedral grains and grain clusters, in } \\
\text { places with granophyric texture. Amph is, in some samples, } \\
\text { anhedral. Oxides are interstitial, although in places they } \\
\text { form subhedral grains }\end{array}$ & $\begin{array}{l}40-50 \% \text { plag }\left(\mathrm{An}_{95}\right) \\
30-50 \% \text { amph } \\
0-20 \% \text { opx } \\
0-10 \% \text { cpx } \\
1-5 \% \text { oxides }\end{array}$ \\
\hline $\begin{array}{l}\text { Relict } \\
\text { troctolite }\end{array}$ & $\begin{array}{l}\text { Medium to coarse grained. Variable mineral assemblages. } \\
\text { Amph-plag dominant. Amph-spinel symplectite common } \\
\text { as rims on opx. When olv is present, corona textures } \\
\text { exist: olv-opx-amph-amph + spin-plag (see Otamendi } \\
\text { et al., 2010). Plag commonly shows a granoblastic texture }\end{array}$ & $\begin{array}{l}50-60 \% \text { plag } \\
\sim 30-40 \% \text { amph } \\
5-10 \% \text { opx } \\
\text { trace spinel, oxides, olv }\end{array}$ \\
\hline $\begin{array}{l}\text { Olivine-- } \\
\text { amphibole } \\
\text { websterite }\end{array}$ & $\begin{array}{l}\text { Medium grained. Olv generally heavily serpentinized. } \\
\text { When plag is present, opx-amph-amph + spinel coronas } \\
\text { separate it from olv. When present, amph is commonly } \\
\text { observed as oikocrysts. Spinel occurs in symplectite with } \\
\text { amph, and as discrete grains }\end{array}$ & $\begin{array}{l}10-50 \% \text { olv }\left(\mathrm{Fo}_{76-82}\right) \\
10-20 \% \text { cpx } \\
10-20 \% \text { opx } \\
1-5 \% \text { green spinel } \\
0-10 \% \text { plag }\left(\mathrm{An}_{97-98}\right) \\
0-25 \% \text { amph }\end{array}$ \\
\hline
\end{tabular}

(Fig. 3b). Mafic inclusions are also observed with sharp contacts and visible reaction rims $(1-2 \mathrm{~cm}$ wide). Metasedimentary xenoliths also occur locally. At one outcrop in Turbante canyon the tonalite unit (see Fig. 3c and d) contains blocks of metasedimentary host-rock, biotite concentrations, round quartz domains $(\sim 5 \mathrm{~cm})$, large $(\sim 5 \mathrm{~cm})$ cordierite crystals sheathed in biotite, plagioclase and quartz, fine-grained dark inclusions, mafic inclusions with multiple, variably coarse growth rims and ultramafic cumulate blocks (decimeter to meter scale).

The tonalite is generally medium to coarse grained $(<1 \mathrm{~cm}$ grain size; see Fig. 4$)$ and is dominated by plagioclase $(50-60 \mathrm{vol}$. \%) with variable amounts of quartz $(10-50 \%)$, orthopyroxene (0-15\%, but typically $\sim 5-10 \%)$, amphibole $(0-20 \%$, but typically $\sim 5 \%)$, biotite $(0-15 \%$, typically $<5 \%)$ and Fe-Ti oxides $(<5 \%)$. Zircon and apatite are observed in trace amounts. The mineral mode can be variable (quartz content in particular) over small distances (decimeters).

\section{Gabbronorite}

Gabbronorite is observed as blob-like inclusions (centimeters to meters) within the tonalite up to massive (hundreds of meters) bodies (Fig. 5). Initially subhorizontal sheets (centimeter to meter thickness) are commonly observed, but modal layering is rare. Centimeter-scale domains of tonalite are observed within the gabbronorite as patches, stringers, layers, and occasionally dikes (centimeter to meter scale) (Fig. 5b). The gabbronorites are typically medium grained $(<0.5 \mathrm{~cm}$ grain size $)$, although finer grained variants are observed as inclusions. Plagioclase is the dominant mineral ( 50 vol. \%) and occurs as granoblastic composite grains in the form of subhedral laths. Clinopyroxene and orthopyroxene are commonly blocky and appear to be the earliest-grown phases. Plagioclase also forms blocky domains overgrown by orthopyroxene, suggesting that in some gabbronorites, orthopyroxene and plagioclase were co-crystallizing. Amphibole forms 5-30 vol. \% of the gabbronorites but is rarely found as discrete, sub- to euhedral grains. Instead, amphibole is most commonly observed as rims on both clino- and orthopyroxene, and as oikocrysts ranging from $\sim 1$ to $5 \mathrm{~cm}$ in diameter (Fig. $5 \mathrm{c}$ ), indicating that it crystallized late with respect to the other phases. The gabbronorites contain variable amounts of orthopyroxene (15-30\%), clinopyroxene (5-20\%), Fe-Ti oxides (1-5\%), and quartz ( $<5 \%)$. Zircon, apatite, green spinel, and pyrite are observed in trace amounts. Mafic pegmatitic pods, typically centimeters to tens of centimeters in width, containing hornblende and plagioclase crystals $(>1 \mathrm{~cm}$ ), are also common (Fig. $5 \mathrm{~d}$ ).

\section{Gabbro dikes}

Fine-grained mafic dikes are observed sporadically throughout the mafic unit; these are generally several centimeters to $\sim 1 \mathrm{~m}$ thick. They are dominated by 

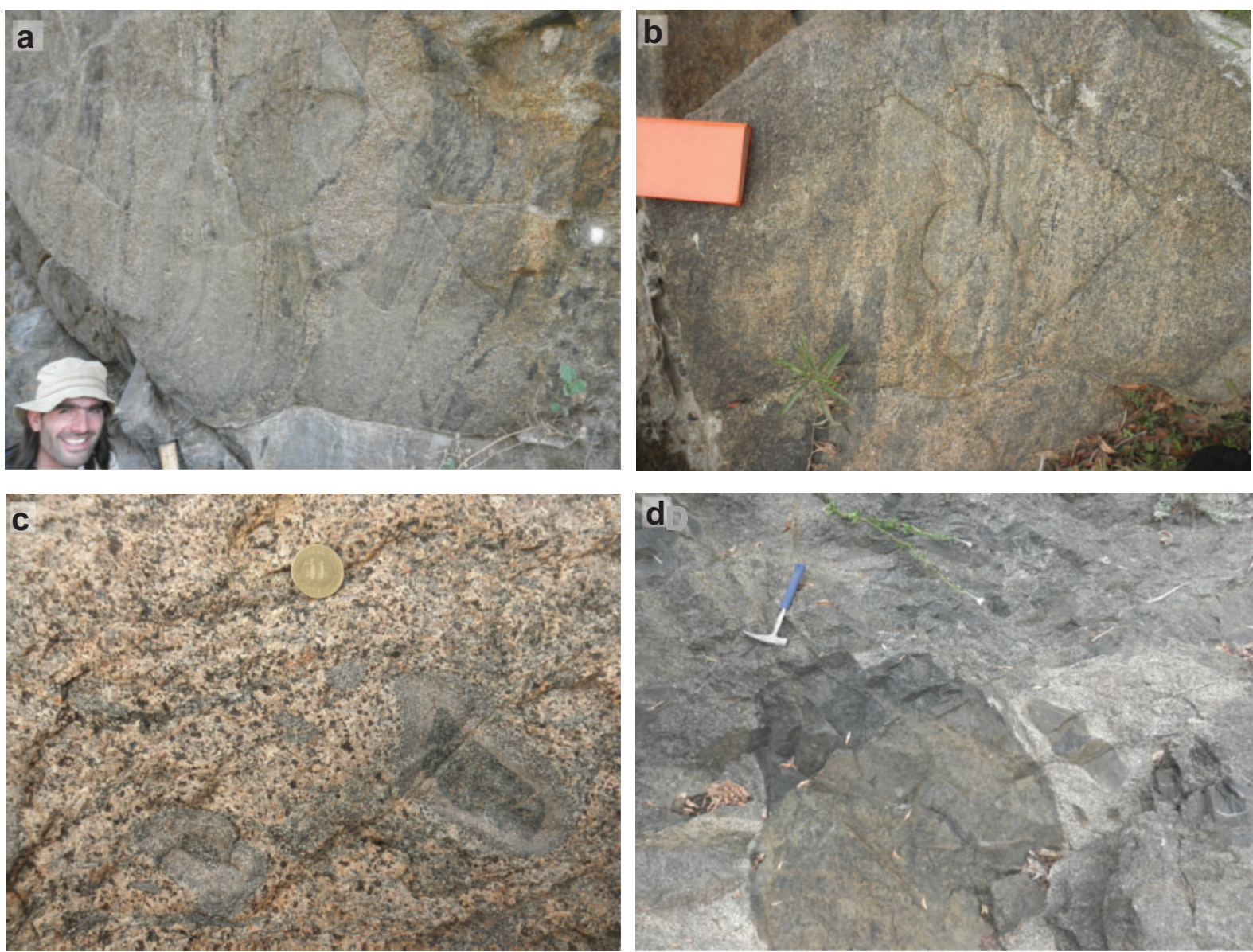

Fig. 3. Field photographs of the tonalite unit showing that it is fairly heterogeneous in outcrop. (a, b) Wispy and sheet-like interlayering with other, modally variable tonalite varieties; mafic inclusions (discrete to vestigial) are common. (c) Mafic inclusions with reaction rims in a tonalite matrix. (d) Large $\sim 1.5 \mathrm{~m}$ block of olivine websterite within an inclusion-rich tonalite. Locally, blocks of other Valle Fertil rock types (metasedimentary host-rock, gabbro) are also preserved within the tonalite.

plagioclase (40-50\%) and amphibole (30-50\%). Unlike in the gabbronorite bodies, where amphibole is found only as rims and oikocrysts, amphibole in the mafic dikes occurs as subhedral grains and grain clusters, with triple-junction boundaries. Orthopyroxene $(0-$ $20 \%$ ), clinopyroxene $(0-10 \%)$ and $\mathrm{Fe}-\mathrm{Ti}$ oxides $(1-5 \%)$ are also present. The dikes always have sharp contacts with the host-rock.

\section{Ultramafic bodies}

The ultramafic rocks of the Valle Fértil mafic zone have been discussed in detail by Otamendi et al. (2010a), but a brief description is warranted here. They comprise clinopyroxene, orthopyroxene, olivine, amphibole, green spinel, and plagioclase. Rock types include olivine websterite, amphibole websterite, troctolite, and anorthosite. These ultramafic bodies range in thickness from tens of meters to $\sim 400 \mathrm{~m}$. They always show gradational transitions into other rock types. For the olivine and amphibole websterite bodies the transition zone is defined by an area in which plagioclase appears and then becomes dominant over an interval of $\sim 10 \mathrm{~m}$, with no sharp contact. When olivine and plagioclase are present together there is an orthopyroxene-amphibole-spinel corona separating the two phases (Meurer \& Claeson, 2002; Otamendi et al., 2010 b), suggesting partial re-equilibration in the presence of a fluid-rich phase. Amphibole is also present as rims on pyroxene and as oikocrysts, suggesting late crystallization with respect to the other phases.

\section{Granodiorite and granite}

Although rare, there are small $\left(<0.5 \mathrm{~km}^{2}\right)$ bodies of granodiorite and granite present in the mafic zone. We have located two $100 \mathrm{~m}$ size silicic blobs (Fig. 2); rare leucogranite dikes are also present. In general, granodiorite and granite are much more abundant farther up the section than in the mafic zone. These silicic rock types are noteworthy for the presence of alkali feldspar ( 15-35 vol. \%), which is not observed in any other rock in the mafic complex. Also present in these rock types are quartz (35-40\%), plagioclase (20-40\%), and biotite $(<5 \%)$.

\section{WHOLE-ROCK COMPOSITIONS}

\section{Major and trace elements}

The rocks of the Valle Fértil mafic zone range from 41 to $75 \% \mathrm{SiO}_{2}$ [Fig. 6; Table 2; see also Supplementary Data 


\section{Tonalite Spectrum}

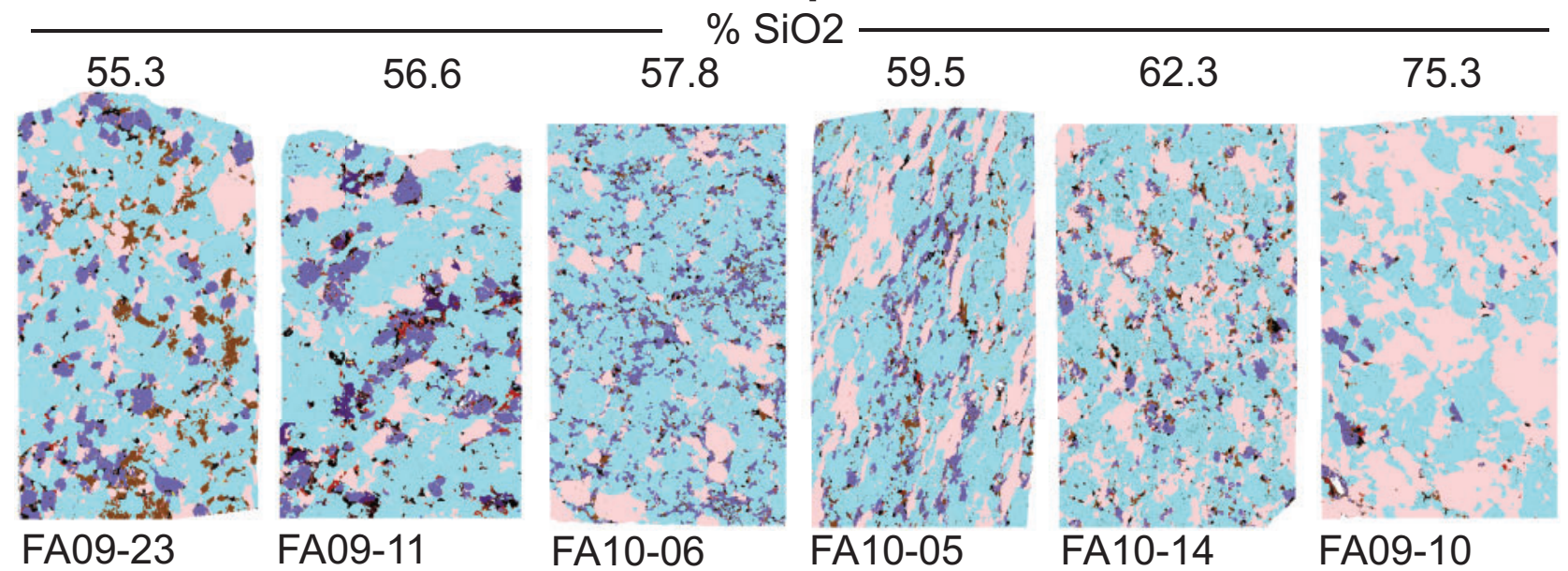

Gabbro Spectrum

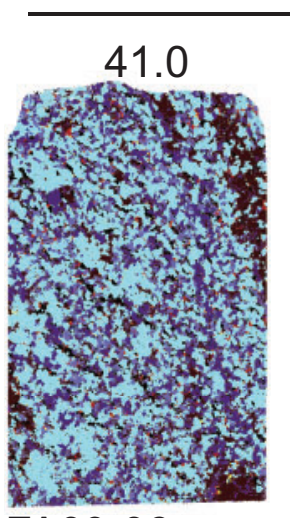

FA09-06

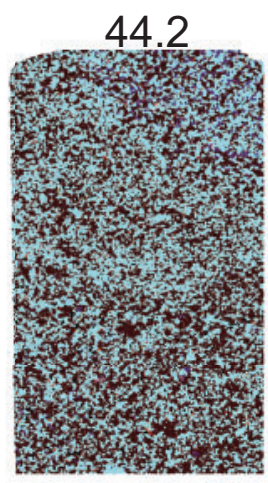

FA09-09
$\% \mathrm{SiO} 2$ 45.5

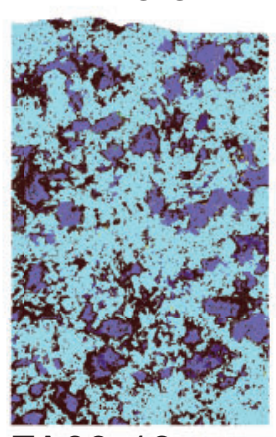

FA09-13

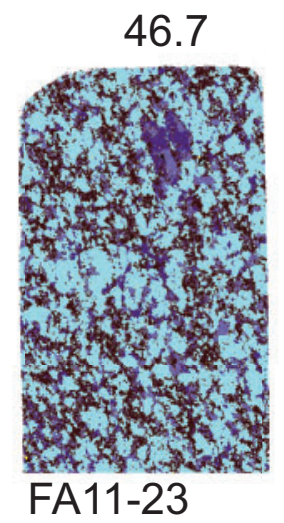

FA11-23

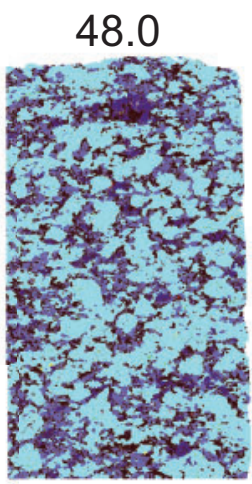

FA09-16

\section{Mineral Color Code}

$\begin{array}{lll}\text { Amph } & \square \text { Qtz } & \text { Olv } \\ \text { Cpx } & \square \text { Plag } & \text { Mag } \\ \text { Opx } & \square \text { Biot } \\ & & \square \mathrm{Im} \\ & 25 \mathrm{~mm} & \end{array}$

Fig. 4. Selected QEMSCAN false-color photomicrographs of SVF mafic zone rocks from the tonalite and gabbronorite spectrum, with $\mathrm{SiO}_{2}$ weight per cent indicated for each sample. Variation in the tonalite spectrum is typically in the quartz (Qtz) and orthopyroxene (Opx) content. Variation in the gabbronorite spectrum is observed in the proportions of clinopyroxene (Cpx), orthopyroxene, amphibole (Amph), and Fe-Ti oxides (Mag, Ilm). Biot, biotite; Olv, olivine; Plag, plagioclase.

(SD) Electronic Appendix 1; supplementary data are available for downloading at http://www.petrology. oxfordjournals.org]. Rocks with less than $\sim 53 \% \mathrm{SiO}_{2}$ have compositional arrays reflecting the variable accumulation and abundance of mineral phases such as olivine, plagioclase, amphibole and $\mathrm{Fe}-\mathrm{Ti}$ oxides. Specifically, olivine-bearing rocks have low $\mathrm{TiO}_{2}, \mathrm{Al}_{2} \mathrm{O}_{3}$ and $\mathrm{CaO}$ and high $\mathrm{MgO}$; plagioclase cumulates have high $\mathrm{CaO}$ and $\mathrm{Al}_{2} \mathrm{O}_{3}$ and low $\mathrm{TiO}_{2}$; rocks with abundant amphibole have high $\mathrm{TiO}_{2}$ and low $\mathrm{SiO}_{2}$; rocks rich in $\mathrm{Fe}-\mathrm{Ti}$ oxides have high $\mathrm{FeO}$ and $\mathrm{TiO}_{2}$ and low $\mathrm{SiO}_{2} \cdot \mathrm{TiO}_{2}$ and $\mathrm{FeO}$ are positively correlated for mafic to ultramafic rocks. For the mafic zone rocks with $>53 \% \mathrm{SiO}_{2}$ (tonalites), $\mathrm{Al}_{2} \mathrm{O}_{3}, \mathrm{FeO}, \mathrm{MgO}, \mathrm{CaO}$ and $\mathrm{TiO}_{2}$ all broadly decrease with increasing $\mathrm{SiO}_{2}$, whereas $\mathrm{Na}_{2} \mathrm{O}$ increases with $\mathrm{SiO}_{2}$ (up to $\sim 62 \% \mathrm{SiO}_{2}$ ), consistent with a differentiation trend. With only a few exceptions, all the tonalites and gabbronorites in the mafic zone have $\mathrm{K}_{2} \mathrm{O}$ below $1 \mathrm{wt} \%$ and most fall between 0.05 and $0.5 \mathrm{wt} \%$.

The $X_{\mathrm{Mg}}\left[\mathrm{Mg} /\left(\mathrm{Mg}+\mathrm{Fe}_{\text {tot }}\right)\right]$ of the mafic to ultramafic rocks ranges from 0.8 to 0.4 . The variation of $X_{\mathrm{Mg}}$ versus $\mathrm{SiO}_{2}$ is Z-shaped, with a positive correlation between $X_{\mathrm{Mg}}$ and $\mathrm{SiO}_{2}$ between $X_{\mathrm{Mg}} \quad 0.65-0.4$ and $\mathrm{SiO}_{2} \sim 50-41 \mathrm{wt} \%$. The highest $X_{\mathrm{Mg}}$ rocks are either 

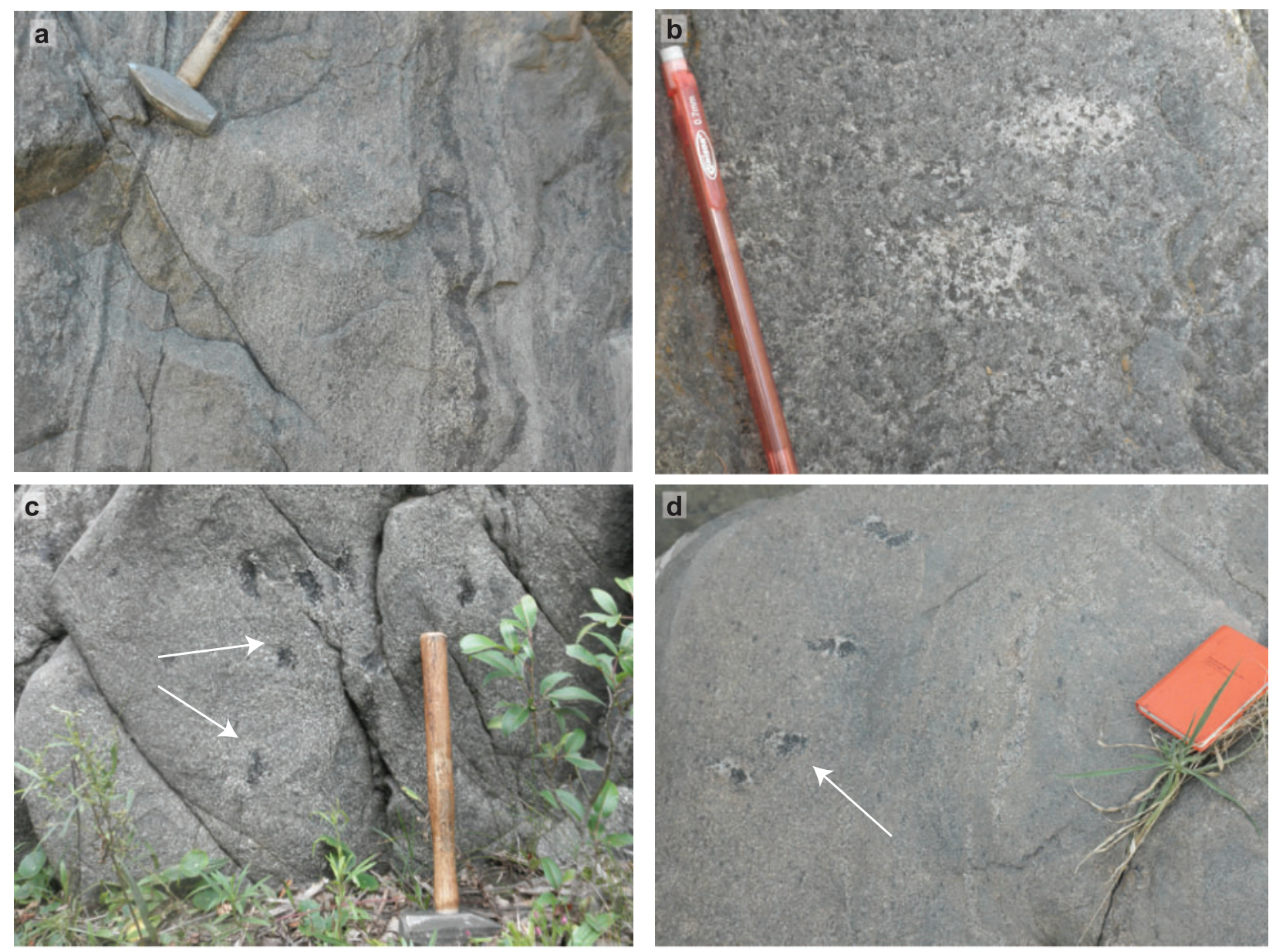

Fig. 5. Field photographs of gabbro to gabbronorite, observed as massive bodies (hundreds of meters) to blob-like inclusions (centimeters) within the tonalite. (a) Wispy layering of gabbronorite varieties. (b) Tonalitic patches within the gabbronorite, which could be silicic segregations. (c) Amphibole oikocrysts, ranging in size from $\sim 1$ to $5 \mathrm{~cm}$ in diameter, are observed in places within the gabbronorite. (d) Typical view of the gabbronorite with silicic wisps and amphibole-rich mafic pegmatites (white arrows).

olivine-bearing or rocks that we interpret to have been originally olivine-bearing but have experienced reactive re-equilibration (relict troctolite with amphibole as the dominant mafic phase). From $X_{\mathrm{Mg}} 0.44$ at $\mathrm{SiO}_{2} 41 \%$, the data trend extends to higher $\mathrm{SiO}_{2}$ with a slight decrease in $X_{\mathrm{Mg}}(0.4$ to $\sim 0.35)$. When the mafic and ultramafic rocks are broken out into groups with respect to their position on this $X_{\mathrm{Mg}}$ vs $\mathrm{SiO}_{2}$ plot, compositional variation can be tracked in several other plots. For example, inflections are observed in $\mathrm{TiO}_{2}, \mathrm{FeO}$ and $\mathrm{Na}_{2} \mathrm{O}$ (all versus $\mathrm{SiO}_{2}$ ).

All mafic zone rocks are enriched in incompatible trace elements with respect to primitive mantle (Sun \& McDonough, 1989; Fig. 7; Table 2). Overall, negative spikes in the high field strength elements $\mathrm{Nb}, \mathrm{Ta}$, Th and $U$ are observed in primitive mantle-normalized trace element patterns, consistent with a subduction signature. For the mafic to ultramafic rocks the abundances of trace elements are consistent with mineral modes. For the tonalites, variation with silica is consistent with the trace element's compatibility in gabbroic mineral phases (Fig. 8). For example, olivine-bearing rocks are enriched in $\mathrm{Ni}$ and $\mathrm{Cr}$ (>400 ppm and
$>500$ ppm respectively); $\mathrm{Ni}$ and $\mathrm{Cr}$ decrease with increasing $\mathrm{SiO}_{2}$ in tonalites; rocks rich in clinopyroxene and $\mathrm{Fe}-\mathrm{Ti}$ oxides have elevated $\mathrm{Sc}$ and $\mathrm{V} \quad(>30$ and $>250$, respectively), whereas Sc and $V$ decrease with increasing $\mathrm{SiO}_{2}$ in tonalites. Most mafic zone rocks (tonalites included) have extremely low concentrations of $\mathrm{Rb}$ and $\mathrm{Ba}$ (mostly $<25 \mathrm{ppm}$ and $<200$ ppm, respectively) in keeping with the low $\mathrm{K}_{2} \mathrm{O}$ of these rocks. However, a few intermediate samples are more enriched in incompatible large ion lithophile elements such as $\mathrm{Rb}, \mathrm{Ba}$ and $\mathrm{Sr}$. Mafic zone rocks have REE concentrations that are enriched $\sim 1$ to 100 times relative to primitive mantle (Fig. 7). With decreasing $X_{M g}$, the mafic-ultramafic rocks become more enriched in the REE. With the exception of some high-SiO ${ }_{2}$ leucogranites, intermediate to silicic rocks from the mafic zone have $\mathrm{Zr} / \mathrm{Hf}$ close to 40 , consistent with chondritic and globally observed values (Hoskin \& Schaltegger, 2003; Claiborne et al., 2006). Most mafic to ultramafic rocks have $\mathrm{Zr} / \mathrm{Hf}$ ranging from 22 to 38 , suggesting some fractionation mechanism. The $\mathrm{Eu} / \mathrm{Eu}^{*}$ values for maficultramafic rocks vary from 0.5 to $2 \cdot 2$, although most samples fall between $\sim 0.7$ and 1.5. The $\mathrm{Eu} / \mathrm{Eu}^{*}$ for more 

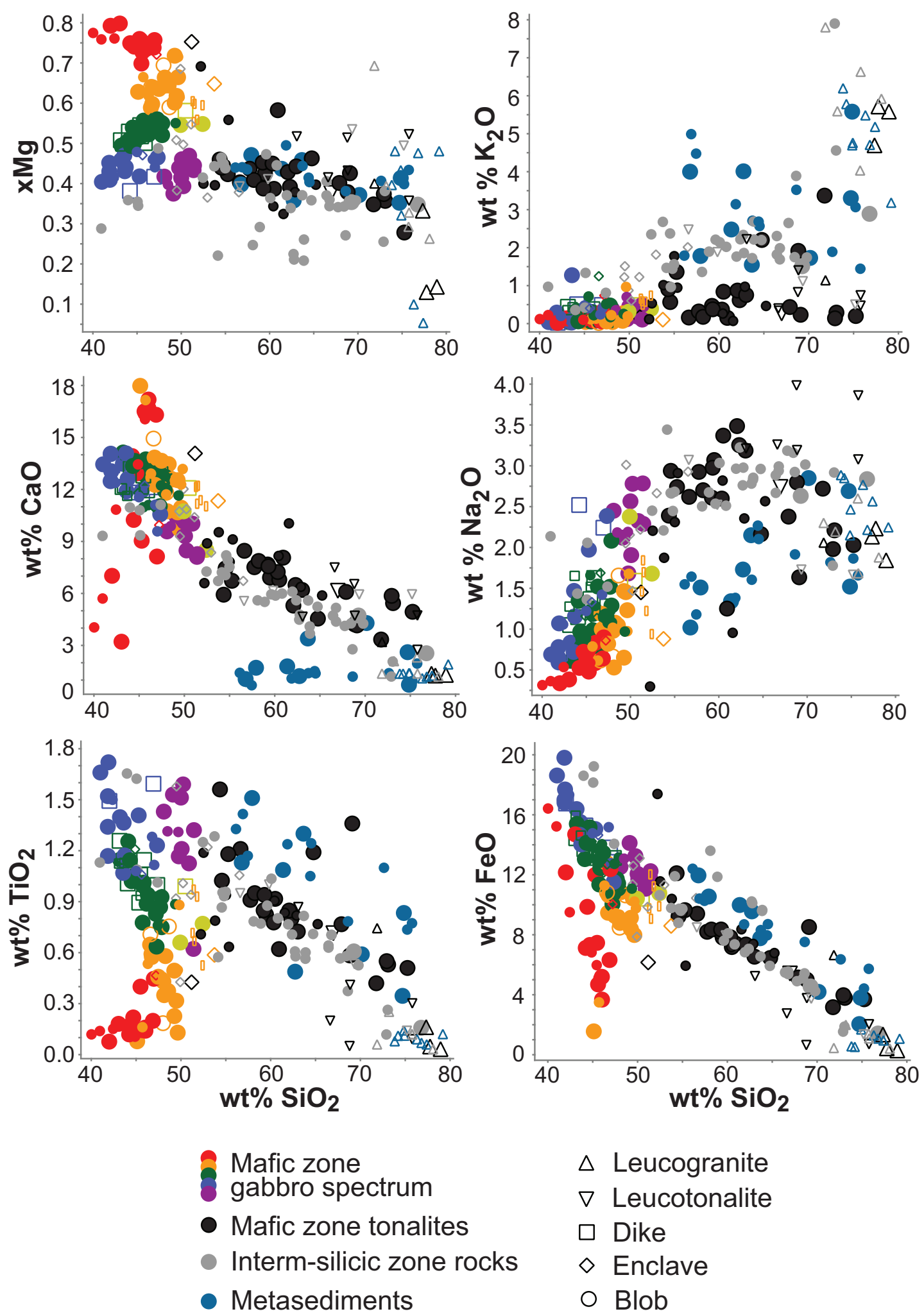
$\triangle$ Leucogranite
$\nabla$ Leucotonalite
$\square$ Dike
$\diamond$ Enclave
○ Blob

Fig. 6. Major element variations vs $\mathrm{SiO}_{2}$ (wt \%) for the SVF. The gabbroic rocks of the mafic zone rocks are color-coded based on their position in the $X_{\mathrm{Mg}}\left[\mathrm{Mg} /\left(\mathrm{Mg}+\mathrm{Fe}_{\mathrm{tot}}\right)\right]$ vs $\mathrm{SiO}_{2}$ plot. Tonalites of the mafic zone are shown in black. Rocks from the intermediate and silicic zones are colored gray; metasedimentary rocks are colored blue. Various other rock types (dike, enclave, blob, leucogranite, leucotonalite) are identified by separate symbols. Large symbols are data from this study, and smaller symbols are data from previous studies (Otamendi et al., 2008, 2009b, 2010a, 2010b, 2012). 
Table 2: Major and trace element compositions of selected samples from the SVF mafic zone

\begin{tabular}{|c|c|c|c|c|c|c|c|}
\hline $\begin{array}{l}\text { Sample: } \\
\text { Lithology: }\end{array}$ & $\begin{array}{l}\text { FA906 } \\
\text { hbl gn }\end{array}$ & $\begin{array}{l}\text { FA907 } \\
\text { qtz-gabbro }\end{array}$ & $\begin{array}{l}\text { FA909 } \\
\text { hbl-gabbro } \\
\text { enclave }\end{array}$ & $\begin{array}{l}\text { FA914 } \\
\text { qtz-norite }\end{array}$ & $\begin{array}{l}\text { FA916 } \\
\text { hbl gn }\end{array}$ & $\begin{array}{l}\text { FA918 } \\
\text { qtz-gabbro }\end{array}$ & $\begin{array}{l}\text { FA1012 } \\
\text { gn }\end{array}$ \\
\hline Canyon: & Usno & Usno & Usno & Usno & Usno & Usno & Las Tunas \\
\hline $\mathrm{SiO}_{2}$ & 41.02 & $49 \cdot 11$ & $44 \cdot 19$ & $50 \cdot 24$ & 48.04 & $52 \cdot 42$ & $43 \cdot 20$ \\
\hline $\mathrm{TiO}_{2}$ & 1.66 & 1.53 & 2.01 & 1.59 & 0.43 & 0.77 & 1.397 \\
\hline $\mathrm{Al}_{2} \mathrm{O}_{3}$ & $17 \cdot 16$ & $17 \cdot 23$ & $17 \cdot 41$ & 19.81 & 19.73 & $17 \cdot 71$ & 16.51 \\
\hline $\mathrm{FeO}_{\mathrm{t}}$ & 18.62 & $14 \cdot 12$ & $15 \cdot 29$ & 11.82 & 8.79 & $10 \cdot 77$ & $16 \cdot 38$ \\
\hline $\mathrm{MgO}$ & 7.09 & 4.75 & $5 \cdot 29$ & $4 \cdot 3$ & 8.89 & $7 \cdot 33$ & 7.78 \\
\hline $\mathrm{CaO}$ & 13.45 & $10 \cdot 46$ & 11.82 & 8.48 & $12 \cdot 82$ & 8.51 & 13.48 \\
\hline $\mathrm{Na}_{2} \mathrm{O}$ & 0.69 & $2 \cdot 12$ & 2.52 & 2.78 & 0.98 & 1.68 & 0.83 \\
\hline $\mathrm{K}_{2} \mathrm{O}$ & 0.04 & 0.09 & 0.51 & 0.44 & 0.13 & 0.39 & 0.09 \\
\hline Total* & 97.54 & 99.42 & 98.68 & 98.94 & 98.41 & 99.17 & 98.20 \\
\hline$X_{\mathrm{Mg}}$ & 0.40 & 0.37 & 0.38 & 0.39 & 0.64 & 0.55 & 0.46 \\
\hline $\mathrm{Ni}$ & $22 \cdot 7$ & 21 & $9 \cdot 1$ & 30.2 & 65.4 & 73.7 & 24.6 \\
\hline $\mathrm{Cr}$ & $4 \cdot 7$ & $19 \cdot 2$ & 0 & $54 \cdot 3$ & 250.6 & 253.6 & $72 \cdot 7$ \\
\hline Sc & $62 \cdot 4$ & $45 \cdot 0$ & $60 \cdot 2$ & $30 \cdot 1$ & 38.6 & 38.2 & $60 \cdot 2$ \\
\hline V & 597.4 & 343 & 332.8 & 235.9 & 227.9 & 302.4 & 541.0 \\
\hline $\mathrm{Ba}$ & 14 & 49 & 115 & 213 & 46 & 121 & $36 \cdot 1$ \\
\hline $\mathrm{Rb}$ & 0.2 & 0.2 & 1.9 & $5 \cdot 3$ & 0.7 & 8.5 & 0.4 \\
\hline $\mathrm{Sr}$ & 159 & 269 & 259 & 249 & 195 & 141 & 185 \\
\hline $\mathrm{Zr}$ & 13 & 231 & 617 & 467 & 18 & 73 & 15 \\
\hline $\mathrm{Y}$ & 8.84 & 35.92 & 52.47 & $16 \cdot 28$ & 8.70 & 24.71 & 11.20 \\
\hline $\mathrm{La}$ & 2.02 & 18.00 & 23.64 & 16.89 & 4.40 & $24 \cdot 12$ & $3 \cdot 10$ \\
\hline $\mathrm{Ce}$ & 5.40 & $48 \cdot 13$ & 60.71 & 35.06 & 9.99 & $49 \cdot 70$ & 7.82 \\
\hline $\operatorname{Pr}$ & 0.82 & $7 \cdot 10$ & 8.94 & 4.44 & 1.36 & 5.92 & $1 \cdot 16$ \\
\hline $\mathrm{Nd}$ & 3.97 & 32.48 & 41.96 & 18.53 & 5.96 & 23.29 & 5.62 \\
\hline $\mathrm{Sm}$ & 1.25 & 7.85 & 11.07 & 3.97 & 1.57 & 5.07 & 1.75 \\
\hline $\mathrm{Eu}$ & 0.46 & 2.02 & 2.85 & 1.64 & 0.51 & 1.24 & 0.67 \\
\hline $\mathrm{Gd}$ & 1.46 & 7.36 & 11.40 & 3.71 & 1.58 & 4.65 & 2.06 \\
\hline $\mathrm{Tb}$ & 0.26 & 1.21 & 1.83 & 0.56 & 0.28 & 0.75 & 0.35 \\
\hline Dy & 1.79 & 7.31 & $10 \cdot 88$ & 3.28 & 1.81 & 4.68 & $2 \cdot 31$ \\
\hline $\mathrm{Ho}$ & 0.35 & 1.45 & $2 \cdot 18$ & 0.66 & 0.36 & 0.96 & 0.48 \\
\hline $\mathrm{Er}$ & 1.01 & 3.94 & $5 \cdot 81$ & 1.82 & 0.99 & 2.75 & 1.27 \\
\hline $\mathrm{Tm}$ & 0.14 & 0.54 & 0.80 & 0.26 & 0.14 & 0.43 & $0 \cdot 18$ \\
\hline $\mathrm{Yb}$ & 0.86 & 3.26 & 4.60 & 1.64 & 0.89 & $2 \cdot 83$ & 1.12 \\
\hline Lu & 0.14 & 0.51 & 0.72 & 0.28 & 0.14 & 0.46 & $0 \cdot 17$ \\
\hline$U$ & 0.01 & 0.08 & 0.40 & 0.27 & 0.03 & 0.61 & 0.05 \\
\hline Th & 0.04 & 0.11 & 1.41 & 0.42 & 0.12 & 4.94 & 0.23 \\
\hline $\mathrm{Hf}$ & 0.49 & 5.40 & $13 \cdot 26$ & 11.48 & 0.59 & $2 \cdot 24$ & 0.51 \\
\hline
\end{tabular}

silicic rocks is much more variable; tonalites range from 0.5 to 4.1 , and leucogranites range from 2.4 to extreme values of 10.5 . In general, elemental trends for the mafic to ultramafic rocks appear to reflect mineral accumulation, whereas the trends for the tonalitic rocks tend to be more linear.

\section{$\mathrm{Sr}, \mathrm{Nd}$ and $\mathrm{Pb}$ isotopes}

The rocks of the Valle Fértil mafic zone exhibit a wide range in isotopic composition (Fig. 9; Table 3); $\mathrm{Sr}, \mathrm{Nd}$, and $\mathrm{Pb}$ isotope ratios fall within the Valle Fértil array reported by Otamendi et al. $(2009 a, 2012)$ and Ducea et al. (2014). With respect to $\mathrm{Sr}$ and Nd isotopes, the most remarkable characteristic of the SVF rocks is that they all deviate strongly from primitive mantle isotopic values. Within the mafic zone, initial ${ }^{87} \mathrm{Sr} /{ }^{86} \mathrm{Sr}$ (age corrected to $475 \mathrm{Ma}$ ) ranges from 0.70525 to 0.71445 , with the mafic to ultramafic rocks spanning the entire range. Tonalites in the mafic zone are more restricted in ${ }^{87} \mathrm{Sr} /{ }^{86} \mathrm{Sr}$, ranging from 0.707531 to 0.712642 (when leucotonalite veins are excluded, the range is even more restricted at $0.709281-0.710635)$. Initial $\varepsilon_{\text {Nd }}$ ranges from 2.2 to -6.5 (corresponding to a range in initial ${ }^{143} \mathrm{Nd} /{ }^{144} \mathrm{Nd}$ of 0.512137-0.511696). As for $\mathrm{Sr}$ isotopes, the mafic and ultramafic rocks also have the largest range in $\varepsilon_{\mathrm{Nd}}(2.2$ to -5.9). Tonalites are restricted to $\varepsilon_{\mathrm{Nd}}$ values of -5.2 to 6.5 (with one leucotonalite vein with $\varepsilon_{\mathrm{Nd}}=-2.5$ ). A negatively correlated array is observed when $\varepsilon_{\mathrm{Nd}}$ is plotted against ${ }^{87} \mathrm{Sr} /{ }^{86} \mathrm{Sr}$; however, variation to the highest ${ }^{87} \mathrm{Sr} /{ }^{86} \mathrm{Sr}$ values $(>0.711)$ is associated with no systematic change in $\varepsilon_{N d}$ values. The samples with the highest $\varepsilon_{\mathrm{Nd}}$ and lowest ${ }^{87} \mathrm{Sr} /{ }^{86} \mathrm{Sr}$ have between 47.5 and $51.5 \mathrm{wt} \% \mathrm{SiO}_{2}$; with the exception of these rocks the mafic zone rocks show generally positive correlations in $\mathrm{SiO}_{2}$ vs ${ }^{87} \mathrm{Sr} /{ }^{86} \mathrm{Sr}$ and negative correlations in $\mathrm{SiO}_{2}$ vs $\varepsilon_{\mathrm{Nd}}$.

Lead isotope compositions (age corrected) in the Valle Fértil mafic zone rocks show only minor variation (Table 3); ${ }^{208} \mathrm{~Pb} /{ }^{204} \mathrm{~Pb}$ ranges from 38.1487 to 38.5526 ; ${ }^{207} \mathrm{~Pb} /{ }^{204} \mathrm{~Pb}$ ranges from 15.6490 to 15.6606 and ${ }^{206} \mathrm{~Pb} /{ }^{204} \mathrm{~Pb}$ ranges from 18.2749 to 18.7270 . The values reported here fall within the range of $\mathrm{Pb}$ isotopic compositions reported by Otamendi et al. (2012) for the Valle Fértil complex. 
Table 2: Continued

\begin{tabular}{|c|c|c|c|c|c|c|c|}
\hline $\begin{array}{l}\text { Sample: } \\
\text { Lithology: }\end{array}$ & $\begin{array}{l}\text { FA2-11-02 } \\
\text { hbl gn }\end{array}$ & $\begin{array}{l}\text { FA2-11-10 } \\
\text { hbl gn } \\
\text { dike }\end{array}$ & $\begin{array}{l}\text { FA2-11-12 } \\
\text { hbl gn }\end{array}$ & $\begin{array}{l}\text { FA2-11-14 } \\
\text { gabbro } \\
\text { enclave }\end{array}$ & $\begin{array}{l}\text { FA2-11-18 } \\
\text { gabbro }\end{array}$ & $\begin{array}{l}\text { FA2-11-22 } \\
\text { hbl gn } \\
\text { dike }\end{array}$ & $\begin{array}{l}\text { FA-12-01 } \\
\text { hbl gn }\end{array}$ \\
\hline Canyon: & Usno & Usno & Usno & Coral Grande & Las Tunas & Coral Grande & Turbante \\
\hline $\mathrm{SiO}_{2}$ & $50 \cdot 89$ & $43 \cdot 16$ & 44.51 & $51 \cdot 18$ & 41.92 & 44.07 & $47 \cdot 33$ \\
\hline $\mathrm{TiO}_{2}$ & 1.126 & 1.258 & 1.020 & 0.426 & 1.720 & 1.007 & 1.231 \\
\hline $\mathrm{Al}_{2} \mathrm{O}_{3}$ & $18 \cdot 46$ & 18.84 & $16 \cdot 34$ & 15.87 & $16 \cdot 90$ & $17 \cdot 75$ & 20.57 \\
\hline $\mathrm{FeO}_{\mathrm{t}}$ & 11.07 & 14.44 & 14.08 & $6 \cdot 16$ & 17.69 & 13.70 & 11.52 \\
\hline $\mathrm{MgO}$ & 5.49 & 8.36 & 9.25 & 10.50 & 6.87 & 8.65 & 5.62 \\
\hline $\mathrm{CaO}$ & $10 \cdot 03$ & $12 \cdot 14$ & 13.65 & 14.08 & 13.05 & 13.20 & 10.57 \\
\hline $\mathrm{Na}_{2} \mathrm{O}$ & $2 \cdot 32$ & 1.05 & 0.79 & 1.45 & 1.07 & 1.15 & $2 \cdot 39$ \\
\hline $\mathrm{K}_{2} \mathrm{O}$ & 0.19 & 0.44 & 0.08 & 0.17 & 0.29 & 0.23 & 0.10 \\
\hline Total* & 99.42 & 97.68 & 99.05 & 98.30 & 96.86 & 97.31 & $99 \cdot 36$ \\
\hline$X_{\mathrm{Mq}}$ & 0.47 & 0.51 & 0.54 & 0.75 & 0.41 & 0.53 & 0.46 \\
\hline $\mathrm{Ni}$ & 32 & 97 & 53 & 187 & 34 & 64 & 22 \\
\hline $\mathrm{Cr}$ & 53 & 106 & 68 & 1339 & 11 & 116 & 38 \\
\hline $\mathrm{Sc}$ & 42.5 & 48.7 & 59.4 & 38.8 & 61.2 & 49.4 & 31.8 \\
\hline V & 296 & 445 & 479 & 168 & 670 & 430 & 282 \\
\hline $\mathrm{Ba}$ & 101 & 96 & 19 & 63 & 75 & 33 & 78 \\
\hline $\mathrm{Rb}$ & 1.4 & $6 \cdot 3$ & 0.3 & $1 \cdot 1$ & 2.9 & 2.2 & 0.3 \\
\hline $\mathrm{Sr}$ & 209 & 1698 & 175 & 95 & 219 & 174 & 277 \\
\hline $\mathrm{Zr}$ & 88 & 17 & 16 & 28 & 32 & 16 & 28 \\
\hline$Y$ & 24.80 & 12.04 & 10.94 & 16.99 & 21.65 & $12 \cdot 48$ & 13.55 \\
\hline $\mathrm{La}$ & 11.36 & 6.20 & 2.92 & $7 \cdot 72$ & 8.92 & 2.42 & $10 \cdot 77$ \\
\hline $\mathrm{Ce}$ & 25.87 & $15 \cdot 34$ & 8.34 & 18.46 & 23.90 & 6.97 & 23.05 \\
\hline $\mathrm{Pr}$ & 3.66 & 2.08 & 1.29 & 2.37 & 3.50 & 1.13 & 3.13 \\
\hline $\mathrm{Nd}$ & $17 \cdot 10$ & $9 \cdot 21$ & $6 \cdot 24$ & 9.65 & 15.84 & $5 \cdot 81$ & $14 \cdot 10$ \\
\hline $\mathrm{Sm}$ & 4.78 & 2.44 & 1.85 & 2.40 & $4 \cdot 15$ & 1.86 & 3.35 \\
\hline $\mathrm{Eu}$ & 1.44 & 0.85 & 0.64 & 0.64 & 1.07 & 0.64 & 1.39 \\
\hline $\mathrm{Gd}$ & 4.98 & 2.51 & 2.08 & 2.68 & $4 \cdot 16$ & $2 \cdot 11$ & 3.31 \\
\hline $\mathrm{Tb}$ & 0.84 & 0.41 & 0.36 & 0.48 & 0.70 & 0.38 & 0.51 \\
\hline Dy & $5 \cdot 13$ & 2.55 & $2 \cdot 26$ & $3 \cdot 12$ & $4 \cdot 28$ & $2 \cdot 45$ & $2 \cdot 89$ \\
\hline $\mathrm{Ho}$ & 1.05 & 0.51 & 0.47 & 0.66 & 0.88 & 0.53 & 0.57 \\
\hline $\mathrm{Er}$ & $2 \cdot 71$ & 1.35 & 1.24 & 1.84 & $2 \cdot 39$ & 1.40 & 1.47 \\
\hline $\mathrm{Tm}$ & 0.38 & 0.19 & 0.18 & 0.28 & 0.34 & 0.21 & 0.20 \\
\hline $\mathrm{Yb}$ & $2 \cdot 27$ & 1.19 & 1.09 & 1.72 & $2 \cdot 10$ & 1.21 & 1.20 \\
\hline Lu & 0.34 & 0.19 & 0.16 & 0.27 & 0.32 & 0.19 & 0.19 \\
\hline U & 0.06 & 0.12 & 0.01 & 0.17 & 0.04 & 0.05 & 0.03 \\
\hline Th & 0.12 & 0.19 & 0.03 & 0.59 & 0.04 & 0.21 & 0.16 \\
\hline $\mathrm{Hf}$ & $2 \cdot 21$ & 0.64 & 0.57 & 1.03 & 1.04 & 0.63 & 0.78 \\
\hline
\end{tabular}

(continued)

\section{MINERAL COMPOSITIONS OF THE MAFIC ZONE ROCKS}

\section{Olivine}

Olivine occurs exclusively in the ultramafic bodies. It typically forms subhedral grains partially reacted to serpentine (Fig. 10). $X_{\mathrm{Mg}}$ of olivine ranges from 0.76 to 0.82 , with less than $1 \%$ variation within samples (Table 4). NiO contents of olivine range from 0.07 to 0.27 wt $\%$ with $\sim 0.05 \mathrm{wt} \%$ variation within samples. No zoning is observed within grains nor is there significant chemical variation within samples. This is consistent with olivine compositional variations from SVF ultramafic bodies higher in the section reported by Otamendi et al. (2010a).

\section{Clinopyroxene}

Clinopyroxene is present in ultramafic bodies, gabbronorites, and some tonalites. It typically occurs as subhedral grains (Fig. 10), although a granoblastic texture is present in places, suggesting some recrystallization. Clinopyroxene is commonly rimmed by amphibole.
Clinopyroxene $X_{\mathrm{Mg}}\left[\mathrm{Mg} /\left(\mathrm{Mg}+\mathrm{Fe}_{\text {tot }}\right)\right]$ ranges from 0.67 to $0.85\left[0.72-0.89\right.$ for $\left.X_{\mathrm{Mg}}=\mathrm{Mg} /\left(\mathrm{Mg}+\mathrm{Fe}^{2+}\right)\right]$, with $<8 \%$ variation within samples (Table 5). Clinopyroxenes from an olivine-bearing cumulate body have the highest $X_{\mathrm{Mg}}$, ranging from 0.83 to 0.85 , consistent with data reported by Otamendi et al. (2010a); $\mathrm{Al}_{2} \mathrm{O}_{3}$ contents of clinopyroxenes vary from 1.5 to $4.2 \mathrm{wt} \%$. For each sample, there is a negative correlation between $\mathrm{Al}_{2} \mathrm{O}_{3}$ and $X_{\mathrm{Mg}}$ (SD Electronic Appendix Fig. 1). This trend is similar to that shown by cpx in gabbronorites from the Chilas complex (Jagoutz et al., 2007), where it was explained as a metamorphic trend owing to a Tschermak exchange of $(\mathrm{Fe}, \mathrm{Mg}) \mathrm{SiAl}_{2}$ during cooling. A similar, but less well-defined, negative correlation also exists between $X_{\mathrm{Mg}}$ and $\mathrm{Na}_{2} \mathrm{O}$ for these samples. Every rim analysis of cpx from sample FA2-11-12 has a slightly higher $X_{\mathrm{Mg}}$ than the corresponding grain interior $\left(X_{\mathrm{Mg}}\right.$ of $\sim 0.77$ vs $0.73-0.75)$.

\section{Orthopyroxene}

Orthopyroxene is observed in ultramafic bodies, gabbronorites and many tonalites. It is typically subhedral 
Table 2: Continued

\begin{tabular}{|c|c|c|c|c|c|c|c|c|}
\hline $\begin{array}{l}\text { Sample: } \\
\text { Lithology: } \\
\text { Canyon: }\end{array}$ & $\begin{array}{l}\text { FA911 } \\
\text { tonalite } \\
\text { Usno }\end{array}$ & $\begin{array}{l}\text { FA912 } \\
\text { tonalite } \\
\text { Usno }\end{array}$ & $\begin{array}{l}\text { FA1005 } \\
\text { tonalite } \\
\text { Usno }\end{array}$ & $\begin{array}{l}\text { FA1019 } \\
\text { tonalite } \\
\text { Usno }\end{array}$ & $\begin{array}{l}\text { FA11-12 } \\
\text { qtz-tonalite } \\
\text { Coral Grande }\end{array}$ & $\begin{array}{l}\text { FA1014 } \\
\text { tonalite } \\
\text { Las Tunas }\end{array}$ & $\begin{array}{l}\text { FA-12-09 } \\
\text { tonalite } \\
\text { Turbante }\end{array}$ & $\begin{array}{l}\text { FA-12-03 } \\
\text { tonalite } \\
\text { Turbante }\end{array}$ \\
\hline $\mathrm{SiO}_{2}$ & 56.64 & 72.93 & 59.48 & 60.93 & $69 \cdot 10$ & $62 \cdot 30$ & 58.22 & 63.02 \\
\hline $\mathrm{TiO}_{2}$ & 1.21 & 0.55 & 0.824 & 0.621 & 1.360 & 0.799 & 0.950 & 0.724 \\
\hline $\mathrm{Al}_{2} \mathrm{O}_{3}$ & $17 \cdot 49$ & 13.09 & 17.88 & 15.55 & 11.33 & 16.58 & 18.06 & $16 \cdot 36$ \\
\hline $\mathrm{FeO}_{\mathrm{t}}$ & $9 \cdot 4$ & 3.97 & 7.82 & 7.41 & 8.52 & 7.06 & 8.35 & 6.54 \\
\hline $\mathrm{MgO}$ & 3.56 & 1.33 & 3.24 & $5 \cdot 80$ & 3.54 & 2.55 & 3.24 & 2.91 \\
\hline $\mathrm{CaO}$ & 8.46 & 5.86 & 6.91 & 8.06 & $4 \cdot 16$ & 6.49 & $7 \cdot 87$ & $6 \cdot 20$ \\
\hline $\mathrm{Na}_{2} \mathrm{O}$ & 2.62 & 1.98 & 3.07 & 1.25 & 1.63 & 3.25 & 2.70 & $3 \cdot 19$ \\
\hline $\mathrm{K}_{2} \mathrm{O}$ & 0.16 & 0.14 & 0.40 & $0 \cdot 15$ & 0.23 & 0.62 & 0.18 & 0.75 \\
\hline Total* & 99.27 & 98.82 & $99 \cdot 13$ & 98.74 & 99.43 & $99 \cdot 32$ & 99.19 & 99.79 \\
\hline$X_{\mathrm{Mg}}$ & 0.40 & 0.37 & 0.42 & 0.58 & 0.43 & 0.39 & 0.41 & 0.44 \\
\hline $\mathrm{Ni}$ & $14 \cdot 8$ & $3 \cdot 7$ & $19 \cdot 3$ & 60.5 & 47 & $10 \cdot 5$ & 14 & 16 \\
\hline $\mathrm{Cr}$ & $18 \cdot 8$ & 7.5 & 34.2 & 219.6 & 107 & $19 \cdot 1$ & 22 & 26 \\
\hline $\mathrm{Sc}$ & $27 \cdot 1$ & 11.4 & 25.5 & 33.6 & 24 & $22 \cdot 6$ & $26 \cdot 0$ & 18.9 \\
\hline $\mathrm{V}$ & 232.7 & 93.3 & $176 \cdot 3$ & 206.4 & 186 & 147.9 & 175 & 156 \\
\hline $\mathrm{Ba}$ & 82 & 67 & 141.4 & 64.5 & 138 & 240.0 & 113 & 519 \\
\hline $\mathrm{Rb}$ & 1.0 & 0.8 & 2.9 & 1.3 & 2.7 & 4.3 & 0.8 & 8.9 \\
\hline $\mathrm{Sr}$ & 249 & 184 & 217 & 140 & 168 & 207 & 267 & 178 \\
\hline $\mathrm{Zr}$ & 260 & 78 & 160 & 41 & 693 & 151 & 84 & 118 \\
\hline $\mathrm{Y}$ & 14.76 & 8.73 & 14.44 & 22.62 & 3.74 & 18.05 & 9.96 & 7.94 \\
\hline La & $10 \cdot 16$ & 7.78 & $14 \cdot 15$ & 12.96 & 22.98 & 18.48 & $13 \cdot 31$ & 17.41 \\
\hline $\mathrm{Ce}$ & $22 \cdot 77$ & $16 \cdot 81$ & 25.93 & 28.72 & 36.97 & $36 \cdot 87$ & 25.44 & 29.28 \\
\hline $\operatorname{Pr}$ & $3 \cdot 12$ & $2 \cdot 15$ & $3 \cdot 13$ & 3.76 & 3.66 & 4.47 & 3.08 & $3 \cdot 23$ \\
\hline $\mathrm{Nd}$ & 14.03 & 8.68 & $13 \cdot 47$ & 15.79 & 12.21 & 18.03 & 12.75 & 12.03 \\
\hline Sm & 3.37 & 1.84 & 3.03 & 4.09 & 1.49 & 4.02 & 2.58 & $2 \cdot 22$ \\
\hline $\mathrm{Eu}$ & 1.44 & 1.03 & 1.94 & 0.99 & 1.56 & 1.52 & 1.44 & 1.25 \\
\hline $\mathrm{Gd}$ & 3.35 & 1.71 & 3.02 & $4 \cdot 10$ & 0.92 & 3.84 & 2.41 & 2.00 \\
\hline $\mathrm{Tb}$ & 0.51 & 0.28 & 0.46 & 0.72 & 0.11 & 0.60 & 0.35 & 0.29 \\
\hline Dy & 3.06 & 1.70 & 2.80 & 4.59 & 0.65 & 3.55 & 2.00 & 1.67 \\
\hline Ho & 0.59 & 0.34 & 0.60 & 0.93 & 0.15 & 0.71 & 0.40 & 0.33 \\
\hline $\mathrm{Er}$ & 1.63 & 0.98 & 1.69 & 2.57 & 0.51 & 1.90 & 1.04 & 0.87 \\
\hline $\mathrm{Tm}$ & 0.23 & 0.15 & 0.26 & 0.37 & $0 \cdot 10$ & 0.27 & 0.14 & $0 \cdot 12$ \\
\hline $\mathrm{Yb}$ & 1.36 & 0.92 & 1.80 & $2 \cdot 30$ & 0.89 & 1.74 & 0.88 & 0.81 \\
\hline $\mathrm{Lu}$ & 0.23 & 0.15 & 0.30 & 0.35 & 0.17 & 0.27 & 0.14 & 0.14 \\
\hline U & 0.15 & 0.16 & 0.17 & 0.17 & 0.30 & 0.24 & 0.05 & 0.19 \\
\hline Th & 0.22 & 0.18 & 0.20 & 0.94 & 0.40 & 0.40 & $0 \cdot 11$ & 0.38 \\
\hline $\mathrm{Hf}$ & $6 \cdot 15$ & $2 \cdot 14$ & 4.04 & 1.24 & $17 \cdot 36$ & 3.82 & 1.97 & 3.06 \\
\hline
\end{tabular}

(continued)

and commonly rimmed by amphibole (Fig. 10). Compositionally, orthopyroxene varies in $X_{\mathrm{Mg}}$ from 0.57 to 0.67 (Table 6) and falls within the hypersthene field (En 55-82). Within single samples, $X_{\mathrm{Mg}}$ variation is $<3 \%$. In contrast to those from cumulate bodies (see Otamendi et al., 2010), orthopyroxenes from the gabbronorites and tonalites of the mafic zone have significantly lower $X_{\mathrm{Mg}}(0.59-0.67$ vs $\sim 0.81$; see SD Electronic Appendix Fig. 1). These compositions fall within the field of arc 'cumulate' orthopyroxene (Spandler et al., 2003).

\section{Amphibole}

Amphibole occurs most commonly (Fig. 10) as rims on pyroxene, and as oikocrysts (ranging in size from $\sim 1$ to $5 \mathrm{~cm}$ in diameter). It is the dominant mafic mineral in gabbroic dikes. Compositionally, the amphiboles straddle the border of the hornblende-tschermakitepargasite field in the classification scheme of Leake et al. (1997) (Table 7; SD Electronic Appendix Fig. 2). Amphibole $X_{\mathrm{Mg}}\left(\mathrm{Fe}\right.$ as $\mathrm{Fe}^{2+}$ ) ranges from 0.67 to 0.83 , although three samples have very similar ranges ( $\sim 0.73$ 0.81), whereas amphibole from FA2-11-02-a fairly deep
( 8 kbar) gabbronorite-has an overall lower $X_{\mathrm{Mg}}$ (0.670.73 , with two analyses at 0.78 and 0.81 ). Other than sample FA2-11-02, the observed compositional variation is minimal within samples.

\section{Plagioclase}

Plagioclase has the largest mode and is absent only in some olivine-bearing rock types. It typically comprises domains $(<1 \mathrm{~cm})$ of many subgrains (Fig. 10) with some granoblastic triple-point boundaries. Plagioclase anorthite contents vary from 74 to $98 \%$ (Table 8; SD Electronic Appendix Fig. 3). However, variation is minimal within samples. Plagioclase from an olivinebearing pyroxenite (FA-09-17) has a composition of $A n_{97-98}$. Three gabbronorite samples (FA-09-16, FA-0906, and FA2-11-02) have plagioclase with $A n_{95-96}$, $A n_{95-96}$, and $A n_{82-89}$, respectively; plagioclase from a mafic dike (FA2-11-10) is $A n_{95-96}$; and plagioclase from two tonalites (FA-11-12 and $F A-10-05)$ is $A n_{74-83}$ and $A n_{73-79}$, respectively. Only minor zoning is observed within single grains. For example, $A n_{76}$ (core) to $A n_{83}$ (rim) from FA-11-12 is the most variation observed in one grain. For comparison, Otamendi et al. (2010a) 
Table 2: Continued

\begin{tabular}{|c|c|c|c|c|c|c|}
\hline $\begin{array}{l}\text { Sample: } \\
\text { Lithology: } \\
\text { Canyon: }\end{array}$ & $\begin{array}{l}\text { FA908 } \\
\text { grano- } \\
\text { diorite } \\
\text { Usno }\end{array}$ & $\begin{array}{l}\text { FA11-13A } \\
\text { alkali granite } \\
\text { leucogr } \\
\text { Coral Grande }\end{array}$ & $\begin{array}{l}\text { FA-12-05 } \\
\text { metapelite } \\
\text { leucogr } \\
\text { Cerro Blanco }\end{array}$ & $\begin{array}{l}\text { FA922 } \\
\text { metapelite } \\
\text { metaseds }\end{array}$ & $\begin{array}{l}\text { FA915 } \\
\text { metapelite } \\
\text { Usno }\end{array}$ & $\begin{array}{l}\text { FA11-11 } \\
\text { metapelite } \\
\text { Coral Grande }\end{array}$ \\
\hline $\mathrm{SiO}_{2}$ & 71.77 & 78.94 & 74.85 & 63.66 & 56.78 & 74.70 \\
\hline $\mathrm{TiO}_{2}$ & 0.42 & $\begin{array}{r}10.54 \\
0.032\end{array}$ & 0.834 & $\begin{array}{c}03.00 \\
1.3\end{array}$ & $\begin{array}{r}1.13 \\
1.13\end{array}$ & 0.346 \\
\hline $\mathrm{Al}_{2} \mathrm{O}_{3}$ & 14.07 & 11.98 & 11.04 & 16.37 & 19.32 & 13.58 \\
\hline $\mathrm{FeO}_{\mathrm{t}}$ & 3.17 & 0.28 & 3.80 & 7.77 & 11.96 & 2.04 \\
\hline $\mathrm{MgO}$ & 0.95 & 0.03 & 1.50 & 3.69 & 4.43 & 0.62 \\
\hline $\mathrm{CaO}$ & 3.34 & 1.30 & 0.73 & 3.4 & 1.06 & 2.61 \\
\hline $\mathrm{Na}_{2} \mathrm{O}$ & 2.72 & 1.84 & 1.52 & $2 \cdot 15$ & 1.02 & 2.69 \\
\hline $\mathrm{K}_{2} \mathrm{O}$ & 3.37 & 5.58 & 5.58 & 1.55 & 4 & $3 \cdot 30$ \\
\hline Total* & 99.70 & 97.99 & 98.18 & 98.23 & 98.61 & 98.48 \\
\hline$X_{\mathrm{Mg}}$ & 0.35 & 0.14 & 0.41 & 0.46 & 0.40 & 0.35 \\
\hline $\mathrm{Ni}$ & 4.4 & 2 & 20 & 57.8 & 248.4 & 10 \\
\hline $\mathrm{Cr}$ & 7.4 & 5 & 53 & 92.8 & 153.7 & 4 \\
\hline Sc & 14.1 & 1 & 9.2 & 11.7 & 17.6 & 6 \\
\hline V & $60 \cdot 8$ & 5 & 75 & 159.8 & 169.1 & 40 \\
\hline $\mathrm{Ba}$ & 482 & 870 & 351 & 787 & 762 & 489 \\
\hline $\mathrm{Rb}$ & 65.4 & 73.8 & 104.8 & 47.0 & 61.7 & 69.9 \\
\hline $\mathrm{Sr}$ & 128 & 93 & 176 & 180 & 147 & 191 \\
\hline $\mathrm{Zr}$ & 123 & 11 & 508 & 422 & 210 & 144 \\
\hline Y & 7.64 & 0.46 & 36.73 & 7.45 & 7.69 & 21.03 \\
\hline $\mathrm{La}$ & 14.25 & 6.64 & 55.96 & 29.35 & 27.35 & $42 \cdot 36$ \\
\hline $\mathrm{Ce}$ & 24.98 & 9.59 & 113.88 & 53.43 & 46.25 & $86 \cdot 31$ \\
\hline $\mathrm{Pr}$ & 2.83 & 0.91 & $13 \cdot 18$ & 5.66 & 4.67 & 9.78 \\
\hline $\mathrm{Nd}$ & 10.96 & 2.92 & 48.82 & 19.71 & 16.39 & 35.97 \\
\hline Sm & $2 \cdot 12$ & 0.39 & 9.55 & 3.22 & 2.66 & 7.06 \\
\hline $\mathrm{Eu}$ & 1.04 & 0.86 & 1.96 & 1.73 & 2.48 & 2.03 \\
\hline $\mathrm{Gd}$ & 1.89 & 0.22 & 7.97 & $2 \cdot 28$ & 2.01 & 5.91 \\
\hline $\mathrm{Tb}$ & 0.27 & 0.02 & 1.24 & 0.28 & 0.28 & 0.86 \\
\hline Dy & 1.52 & $0 \cdot 10$ & 7.26 & 1.48 & 1.53 & 4.61 \\
\hline $\mathrm{Ho}$ & 0.31 & 0.02 & 1.44 & 0.30 & 0.32 & 0.83 \\
\hline $\mathrm{Er}$ & 0.82 & 0.04 & 3.88 & 0.84 & 0.95 & 2.04 \\
\hline $\mathrm{Tm}$ & 0.12 & 0.01 & 0.57 & 0.13 & 0.15 & 0.26 \\
\hline $\mathrm{Yb}$ & 0.76 & 0.04 & 3.64 & 0.98 & 1.05 & 1.49 \\
\hline $\mathrm{Lu}$ & 0.13 & 0.01 & 0.57 & 0.20 & 0.19 & 0.24 \\
\hline U & 0.25 & 0.10 & 3.05 & 0.47 & 0.37 & 0.54 \\
\hline Th & 0.33 & 0.93 & 19.15 & 2.55 & 4.52 & 13.92 \\
\hline $\mathrm{Hf}$ & 3.23 & 0.42 & 13.45 & 11.41 & 5.92 & 3.86 \\
\hline
\end{tabular}

(continued)

reported SVF plagioclase compositions from an ultramafic cumulate body ranging from $\mathrm{An}_{94}$ to $A n_{99}$; plagioclase from a hornblende gabbronorite yielded compositions ranging from $\mathrm{An}_{89}$ to $\mathrm{An}_{92}$.

\section{DISCUSSION}

\section{Mafic zone parent magma}

High- $X_{\mathrm{Mg}}$ rocks exposed within the mafic zone constrain the composition of the parental magma to the Valle Ferrtil mafic complex. The rocks with the highest $X_{\mathrm{Mg}}$ are olivine websterites and relict troctolites and occur as lenses or bodies meters to hundreds of meters thick. Olivines from the ultramafic cumulates $\left(X_{\mathrm{Mg}}=0.77-0.82\right.$; Otamendi et al., 2010a) are in equilibrium with a crystallizing melt with $X_{\mathrm{Mg}}$ of $0.54-0.62$ [assuming a $K_{\mathrm{D}}{ }^{\text {(ol-liq) }}(\mathrm{Fe}-\mathrm{Mg})=0.32$ (Roeder \& Emslie, 1970)]. These calculated melt $X_{\mathrm{Mg}}$ values are too low to be in equilibrium with the mantle, suggesting that the parental magmas experienced some differentiation prior to crystallizing the ultramafic cumulates. Basal dunites and pyroxenites observed in the Kohistan and
Talkeetna complexes (DeBari \& Mortensen, 1994; Jagoutz et al., 2006) are perhaps analogs for cumulates crystallized from parental magmas prior to ascent to the level exposed at the SVF.

Otamendi et al. (2010a), in their study of a Valle Fértil ultramafic cumulate body, demonstrated that pyroxene crystallization temperatures were $\sim 1200^{\circ} \mathrm{C}$, with olivine having crystallized at even higher temperatures. Notably, the lithologies of the ultramafic cumulates are indicative of an $\mathrm{H}_{2} \mathrm{O}$-undersaturated crystallizing magma. Amphibole is present interstitially or as overgrowths or reaction rims on pyroxenes, indicating that the water content of the magma was not sufficient to crystallize amphibole at an early stage. Based on this, Otamendi et al (2010a) inferred an initial water content of $\sim 1-2 \%$.

\section{Magma evolution recorded in the mafic zone gabbronorite cumulates}

With respect to major and trace elements, the mafic to ultramafic rocks exhibit subtle trends. The low- $\mathrm{SiO}_{2}$ compositions of the gabbronorites are not 
Table 2: Continued

\begin{tabular}{|c|c|c|c|c|c|c|}
\hline $\begin{array}{l}\text { Sample: } \\
\text { Lithology: } \\
\text { Canyon: }\end{array}$ & $\begin{array}{l}\text { FA917 } \\
\text { amp-olv-norite } \\
\text { Usno }\end{array}$ & $\begin{array}{l}\text { FA-12-07 } \\
\text { websterite } \\
\text { Turbante }\end{array}$ & $\begin{array}{l}\text { FA-12-08 } \\
\text { troctolite } \\
\text { Turbante }\end{array}$ & $\begin{array}{l}\text { FA-12-13 } \\
\text { websterite } \\
\text { Algarrobo }\end{array}$ & $\begin{array}{l}\text { FA2-11-01B } \\
\text { relict troctolite } \\
\text { Usno }\end{array}$ & $\begin{array}{l}\text { FA2-11-13 } \\
\text { relict troctolite } \\
\text { Coral Grande }\end{array}$ \\
\hline $\mathrm{SiO}_{2}$ & $45 \cdot 28$ & $\begin{array}{c}43.05 \\
0.181\end{array}$ & $\begin{array}{c}42.00 \\
0.076\end{array}$ & $\begin{array}{c}46.98 \\
0.446\end{array}$ & $\begin{array}{c}45.60 \\
0.144\end{array}$ & $\begin{array}{c}46.03 \\
0.159\end{array}$ \\
\hline $\mathrm{Al}_{2} \mathrm{O}_{3}$ & $11 \cdot 19$ & 5.47 & $12 \cdot 13$ & 8.94 & 25.15 & $\begin{array}{l}0.159 \\
23.09\end{array}$ \\
\hline $\mathrm{FeO}_{\mathrm{t}}$ & 11.99 & 14.70 & $12 \cdot 16$ & $12 \cdot 38$ & 4.69 & $5 \cdot 19$ \\
\hline $\mathrm{MgO}$ & $21 \cdot 27$ & 32.60 & 26.05 & 21.64 & 7.02 & 8.07 \\
\hline $\mathrm{CaO}$ & 9.04 & 3.21 & 7.02 & 8.13 & 16.51 & 16.69 \\
\hline $\mathrm{Na}_{2} \mathrm{O}$ & 0.48 & 0.38 & 0.34 & 0.90 & 0.62 & 0.59 \\
\hline $\mathrm{K}_{2} \mathrm{O}$ & 0.32 & 0.14 & 0.02 & 0.32 & 0.18 & 0.08 \\
\hline Total* & 98.06 & 93.64 & 94.41 & 99.02 & 98.71 & 97.44 \\
\hline$X_{\mathrm{Mg}}$ & 0.76 & 0.80 & 0.79 & 0.76 & 0.73 & 0.73 \\
\hline $\mathrm{Ni}$ & 453.8 & 742 & 440 & 527 & 85 & 84 \\
\hline $\mathrm{Cr}$ & $926 \cdot 1$ & 1175 & 368 & 2292 & 445 & 545 \\
\hline Sc & 28.5 & $16 \cdot 4$ & 11.4 & 33.6 & 25.4 & 29.4 \\
\hline V & 112.9 & 85 & 50 & 175 & 80 & 96 \\
\hline $\mathrm{Ba}$ & 52 & 32 & 9 & 140 & 81 & 24 \\
\hline $\mathrm{Rb}$ & $10 \cdot 6$ & 3.3 & 0.5 & $10 \cdot 7$ & 4.0 & 1.9 \\
\hline $\mathrm{Sr}$ & 90 & 47 & 90 & 103 & 218 & 208 \\
\hline $\mathrm{Zr}$ & 17 & 14 & 3 & 31 & 6 & 6 \\
\hline Y & 5.89 & 4.57 & 1.20 & 9.57 & 3.02 & $3 \cdot 30$ \\
\hline $\mathrm{La}$ & 2.64 & $2 \cdot 35$ & 0.43 & 5.97 & 1.03 & 1.22 \\
\hline $\mathrm{Ce}$ & 6.79 & 5.47 & 0.95 & 11.51 & $2 \cdot 38$ & 2.80 \\
\hline $\operatorname{Pr}$ & 0.94 & 0.76 & 0.13 & 1.45 & 0.32 & 0.39 \\
\hline $\mathrm{Nd}$ & $4 \cdot 24$ & $3 \cdot 37$ & 0.58 & $6 \cdot 29$ & 1.56 & 1.80 \\
\hline Sm & 1.11 & 0.88 & $0 \cdot 18$ & 1.60 & 0.47 & 0.55 \\
\hline Eu & 0.29 & 0.21 & 0.09 & 0.49 & 0.24 & 0.22 \\
\hline $\mathrm{Gd}$ & $1 \cdot 13$ & 0.88 & 0.20 & 1.81 & 0.53 & 0.62 \\
\hline $\mathrm{Tb}$ & 0.19 & $0 \cdot 15$ & 0.03 & 0.31 & 0.09 & 0.11 \\
\hline Dy & $1 \cdot 18$ & 0.91 & 0.22 & 1.88 & 0.60 & 0.65 \\
\hline Ho & 0.24 & 0.18 & 0.05 & 0.39 & 0.13 & 0.14 \\
\hline Er & 0.64 & 0.51 & 0.13 & 1.08 & 0.34 & 0.36 \\
\hline Tm & 0.09 & 0.08 & 0.02 & 0.15 & 0.05 & 0.05 \\
\hline $\mathrm{Yb}$ & 0.57 & 0.47 & 0.14 & 0.96 & 0.30 & 0.32 \\
\hline Lu & 0.08 & 0.07 & 0.02 & 0.14 & 0.05 & 0.05 \\
\hline$U$ & 0.22 & 0.13 & 0.02 & 0.49 & 0.04 & 0.07 \\
\hline Th & 0.86 & 0.57 & 0.09 & $2 \cdot 26$ & 0.14 & 0.22 \\
\hline $\mathrm{Hf}$ & 0.61 & 0.44 & 0.09 & 0.84 & 0.20 & 0.21 \\
\hline
\end{tabular}

*Pre-normalization totals.

gn, gabbronorite.

representative of melts and they appear to be the cumulate product of a liquid line of descent (sensu lato). This evolution is illustrated by the Z-shaped pattern observed in the $X_{\mathrm{Mg}}$ vs $\mathrm{SiO}_{2}$ plot (see Fig. 6). Wholerock $X_{\mathrm{Mg}}$ values of the mafic rocks decrease from 0.8 to $\sim 0.65$ with increasing $\mathrm{SiO}_{2}$, at which point the trend turns and $\mathrm{SiO}_{2}$ decreases with decreasing $X_{\mathrm{Mg}}$. A similar inflection is also observed in the $\mathrm{SiO}_{2}$ vs $\mathrm{TiO}_{2}$ trend. This and other compositional ( $\mathrm{FeO}, \mathrm{V})$ and modal data suggest the inflection of the $\mathrm{SiO}_{2}$ vs $X_{\mathrm{Mg}}$ (and $\mathrm{TiO}_{2}$ ) trend corresponds to the onset of Fe-Ti oxide crystallization and accumulation. At $X_{\mathrm{Mg}}$ of $\sim 0.4$ and $42 \%$ $\mathrm{SiO}_{2}$, there is another inflection in the trend. The samples that fall near this second inflection are marked by the greatest abundances of Fe-Ti oxides ( 5-8 vol. \%), and, correspondingly, the highest $\mathrm{FeO}(\sim 20 \mathrm{wt} \%)$ and $\mathrm{TiO}_{2}(\sim 1.8 \mathrm{wt} \%)$ concentrations. From this point the compositions range to higher $\mathrm{SiO}_{2}$ values with slightly decreasing $X_{\mathrm{Mg}}$-a typical trend for intermediate arc rocks. Jagoutz et al. (2011) reported a similar Z-shaped $X_{\mathrm{Mg}}$ vs $\mathrm{SiO}_{2}$ trend for the Southern Plutonic Complex of the Kohistan batholith. There, the compositional spectrum begins at higher $X_{\mathrm{Mg}}(\sim 0.9)$ and some rock types are garnetiferous (Jagoutz et al., 2007, 2011).

Clinopyroxene compositions yield additional evidence for the evolution of the crystallizing magma. As with olivine, the $X_{\mathrm{Mg}}$ of clinopyroxene can be used to estimate the $X_{\mathrm{Mg}}$ of the melt with which it is in equilib-

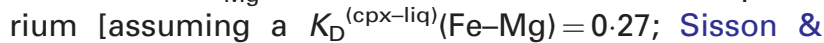
Grove, 1993; Pichavant \& Macdonald, 2007]. For the SVF rocks, clinopyroxene $X_{\mathrm{Mg}}$ (and calculated melt $X_{\mathrm{Mg}}$ ) decreases with whole-rock $X_{\mathrm{Mg}}$, but the wholerock and calculated melt $X_{\mathrm{Mg}}$ do not have a 1:1 correlation (Fig. 11). Instead, rocks with high $X_{M g}(>0.45)$ fall above the 1:1 line-with whole-rock $X_{\mathrm{Mg}}$ being greater than calculated melt $X_{\mathrm{Mg}}$-suggesting that they accumulated Mg-rich phases (such as olivine and orthopyroxene) in the melt from which they crystallized. Notably, several samples with $<45$ wt $\% \mathrm{SiO}_{2}$ have calculated melt $X_{\mathrm{Mg}}<0.45$ (and whole-rock $X_{\mathrm{Mg}}$ of $<0.45$ ), indicating that they probably crystallized from a more evolved magma.

The geochemical trends and the clinopyroxene compositions indicate that the gabbronorite suite is a 


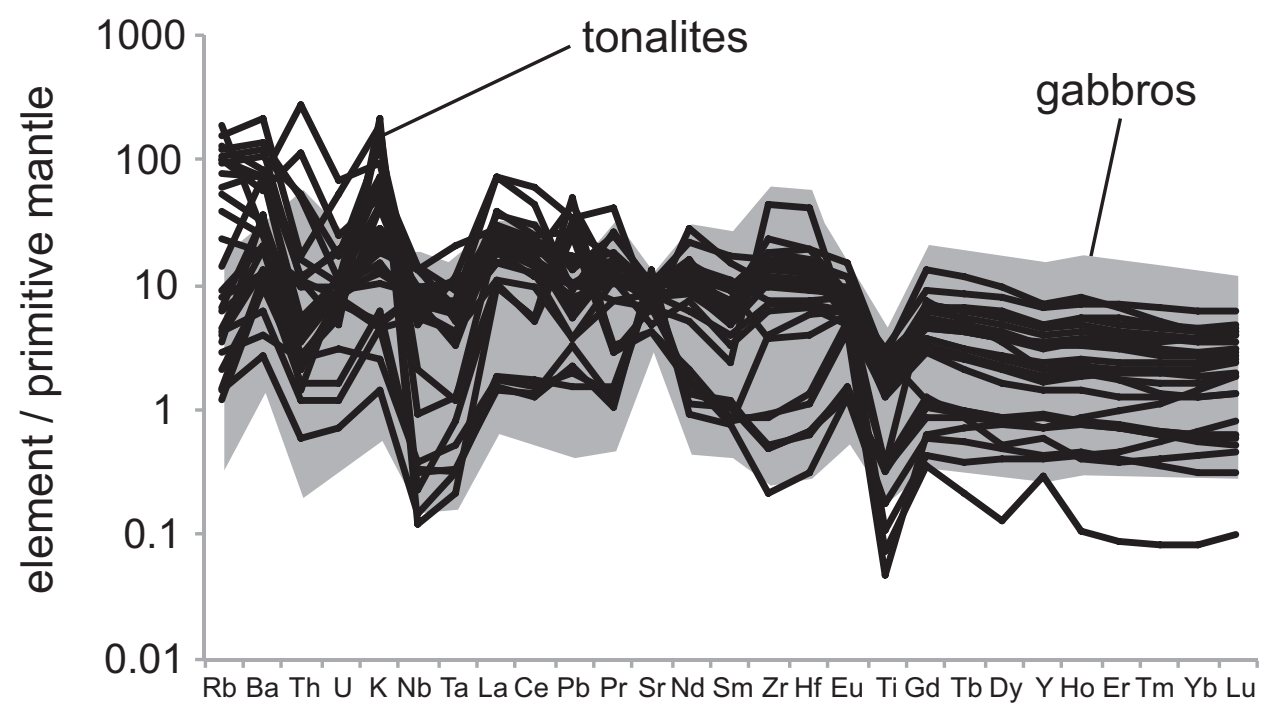

Fig. 7. Primitive mantle-normalized trace element patterns for the SVF tonalites. Primitive mantle values from Sun \& McDonough (1989). The gray field represents the range of compositions of SVF gabbros $\left(<51 \% \mathrm{SiO}_{2}\right)$. There is overall similarity between the patterns for the tonalites and the more mafic rocks, although some tonalites are more enriched in elements that are incompatible with a gabbroic mineral assemblage ( $R b, B a, K, L R E E)$.

cumulate or residual assemblage. We rarely observe cumulus textures such as monomineralic banding or size sorting. However, primary igneous features such as inclusions, small pegmatites, veinlets, and local modal layering are preserved; consequently, subsequent metamorphic re-equilibration was limited, probably to the grain scale. It is also likely that repeated disruption and mixing of the crystal mush by open-system processes disrupted the gradual accumulation of crystals from a stagnant or weakly convecting melt.

\section{Tonalites in the mafic zone}

Tonalites form a significant portion ( $40 \%$ by area) of the SVF mafic zone. There are many subtle differences between the tonalites of the SVF mafic zone and those higher in the section in the intermediate and silicic zones. The most significant difference is that the mafic zone tonalites have a low $\mathrm{K}_{2} \mathrm{O}$ concentration ( $<1 \mathrm{wt} \%$, with many less than $0.5 \mathrm{wt} \%$; see Fig. 6 ) with respect to the silicic zone tonalites $\left(\sim 2 \mathrm{wt} \% \mathrm{~K}_{2} \mathrm{O}\right)$. In general, the tonalites from the mafic zone are also less silicic than those higher in the section. Additionally, at any given silica content, mafic zone tonalites tend to have slightly higher concentrations of $\mathrm{MgO}$ and $\mathrm{CaO}$, and lower concentrations of $\mathrm{Rb}, \mathrm{Ba}$, and light REE (LREE) (see Figs 6 and 8 ).

Tonalites in the SVF mafic zone are heterogeneous, complexly interlayered and gradational into the gabbronorites. Tonalitic material is also present as stringers, patches, and veins in many of the gabbronoritic rocks (see Fig. 3). One sample of such a leucotonalite vein (FA-12-14) is indistinguishable from the more massive, low-K tonalitic domains. We were unable to identify many field relations that unambiguously demonstrate the process by which the magma differentiated-only that tonalite coexists with gabbronorite in outcrop and that ostensibly the two were segregated, or in the process of being segregated, from one another (Zavala et al., 2011; Putirka et al., 2014). Below, we explore a petrogenetic model in which we relate the gabbronorites and the tonalites through fractional crystallization.

\section{Petrogenetic model}

Comprehensive petrogenetic models for the Sierra Valle Fertíl suite (mafic to felsic rocks) have been previously presented, although these models do not adequately describe the compositional variation of all the SVF rocks (Otamendi et al., 2009a, 2010a, 2012). Because the compositions of the low-K tonalites of the SVF mafic zone are distinct from those found elsewhere in the suite, we treat these data separately. Here we provide a fractional crystallization model for major and trace elements to explore the genetic relationship of mafic zone gabbronorites and tonalites. From a starting composition cumulate compositions are removed in steps. The cumulate composition is changed with each fractionation step. This model is explicitly linked to the geology of the SVF: the cumulate composition removed at any given step is a calculated average composition of a mafic to ultramafic subgroup from the SVF mafic complex. Essentially, observed whole-rock compositions are used for cumulates, rather than inferred crystallizing assemblages and fixed distribution coefficients (see Jagoutz, 2010; Bucholz et al., 2014).

The starting composition for the SVF model (see Table 9) was calculated by averaging three SVF mafic rocks with $X_{\mathrm{Mg}}$ from 0.55 to 0.6. One of these (FA-11-01) is a mafic dike; the other two (FA-12-17 and FA-09-18) are mafic rocks with relatively non-cumulate compositions $\left(\sim 50-52 \% \mathrm{SiO}_{2}, \mathrm{Eu} / \mathrm{Eu}^{*}=0.7-0.75\right)$. This starting composition is consistent with olivine and clinopyroxene compositions that suggest that the parental magma 

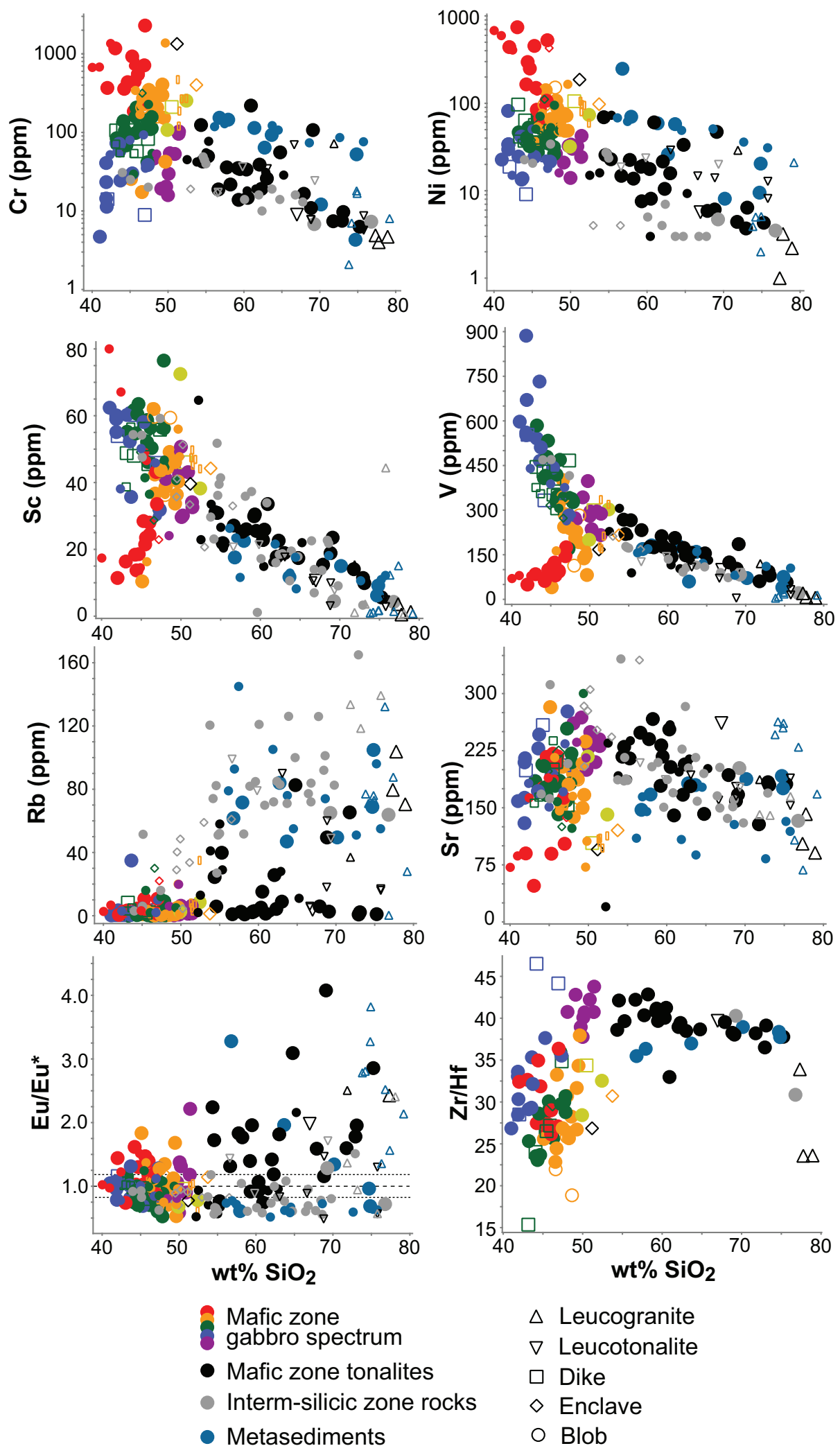
$\Delta$ Leucogranite
$\nabla$ Leucotonalite
$\square$ Dike
$\diamond$ Enclave
O Blob

Fig. 8. Trace element variations vs $\mathrm{SiO}_{2}$ for the SVF. The gabbroic rocks of the mafic zone rocks are color-coded as in Fig. 6 . Tonalites of the mafic zone are shown in black. Rocks from the intermediate and silicic zones are colored gray; metasedimentary rocks are colored blue. Other types of sample (dike, enclave, blob, leucogranite, leucotonalite) are identified by separate symbols. Large symbols are data from this study, and smaller symbols are samples from previous studies (Otamendi et al., 2008, 2009b, 2010a, 2010b, 2012) 

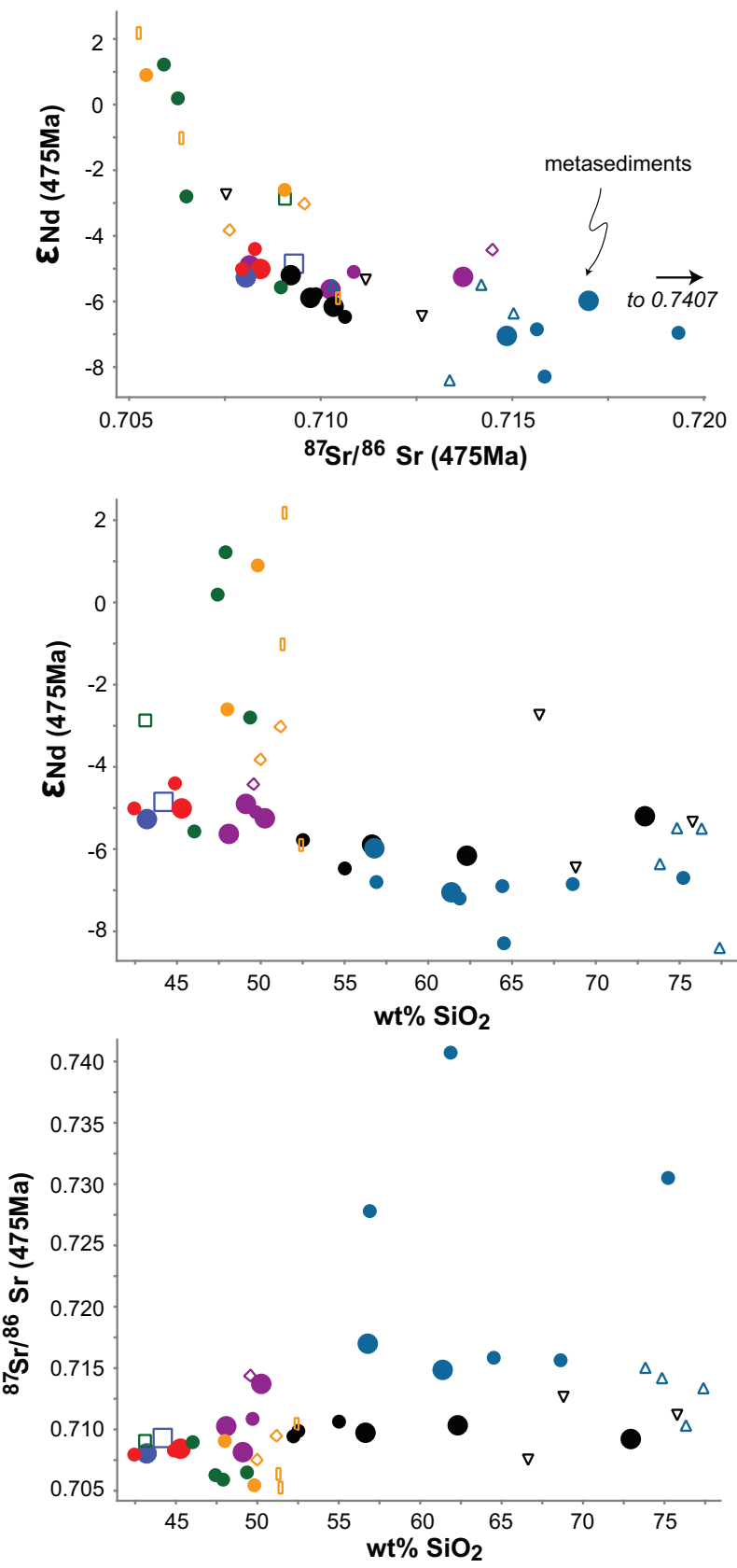

Fig. 9. Isotopic compositions $\left({ }^{87} \mathrm{Sr} /{ }^{86} \mathrm{Sr}\right.$ and $\varepsilon_{\mathrm{Nd}}$, both age-corrected to $475 \mathrm{Ma}$ ) of the SVF mafic zone rocks and associated metasediments. Data for each isotopic system are also plotted vs $\mathrm{SiO}_{2}$. It should be noted that even the most silica-deficient rocks are significantly contaminated (high ${ }^{87} \mathrm{Sr} /{ }^{86} \mathrm{Sr}$, low $\varepsilon_{\mathrm{Nd}}$ ) with respect to primitive mantle. For the magmatic rocks there is no significant correlation of either isotopic system with $\mathrm{SiO}_{2}$. Symbols as in Fig. 8.

to the exposed SVF magma system was not a mantleequilibrated primitive basaltic melt. The concentrations of several trace elements (notably Sr, Y, Sc, and REE) were adjusted from the calculated starting composition to provide a better fit for the model, although these adjustments were always within the range of concentrations for the three rocks used for the starting composition average.
From the starting composition, five distinct cumulate compositions were progressively subtracted over five steps to derive residual liquids (the 'liquid line of descent'; Fig. 12). The mass-balance equation used to calculate the concentration for any given element-major or trace-at each step is

$$
D=(M-X C) /(1-X)
$$

where $D$ is the elemental concentration in the daughter magma, $M$ is the elemental concentration in the starting magma, $C$ is the elemental concentration of the cumulate removed during that step, and $X$ is the fraction of cumulate removed during the step (e.g. $X=0.2$ for $20 \%$ removal). Each of the five cumulate compositions used in the model is an average of one of the compositional groups identified on the $X_{\mathrm{Mg}}$ vs $\mathrm{SiO}_{2}$ trend (Fig. 13; Table 9). The model proceeds by first subtracting out a chosen percentage of cumulate group 1. From the daughter magma derived from that first step, a percentage of cumulate group 2 is subtracted. From that daughter magma, a percentage cumulate group 3 is subtracted, and so on.

From the starting composition the best-fit model subtracts, stepwise, $20 \%$ of cumulate group 1, $15 \%$ of group $2,10 \%$ of group $3,30 \%$ of group 4 , and $30 \%$ of group 5 , for a maximum of $70 \%$ crystals removed relative to the original magma $(F=0.3)$. The most evolved daughter magma produced in this model is a tonalite with $62 \% \mathrm{SiO}_{2}$. This fractionation model is consistent with the production of the $<62 \% \quad \mathrm{SiO}_{2}$ tonalites observed in the field. One important distinction of this model is that this fractionation pathway is forced to produce the same 'cumulate line of descent', consistent with compositional data from the SVF cumulates (ultramafic cumulates and hornblende gabbronorites). For the model to fit, the crystallization sequence must proceed in its correct order, regardless of where it is stopped (for example, first removing $20 \%$ of cumulate group 1 , then $15 \%$ of cumulate group 2 , then $10 \%$ of cumulate group 3 , etc.).

\section{Discussion of the petrogenetic model}

Much of the SVF mafic zone compositional array below $62 \mathrm{wt} \% \mathrm{SiO}_{2}$ is consistent with an origin by fractional crystallization in which the mafic rocks are the cumulate assemblages and the silicic rocks are the daughter magmas. However, it is difficult to field-test the cumulate volumes that our model implies. With the exception of the olivine-bearing samples, the cumulate rocks from the different groups are all gabbronorites and appear very similar in hand sample, making it extremely difficult to distinguish between the gabbronorite varieties in the field. For this model, the cumulate volumes-per cent fractionated-were chosen so as to generate the best fit for the residual liquid to the tonalites within the mafic zone. We do not, then, place special importance on these exact volumes of cumulates, although they do provide the best fit. 


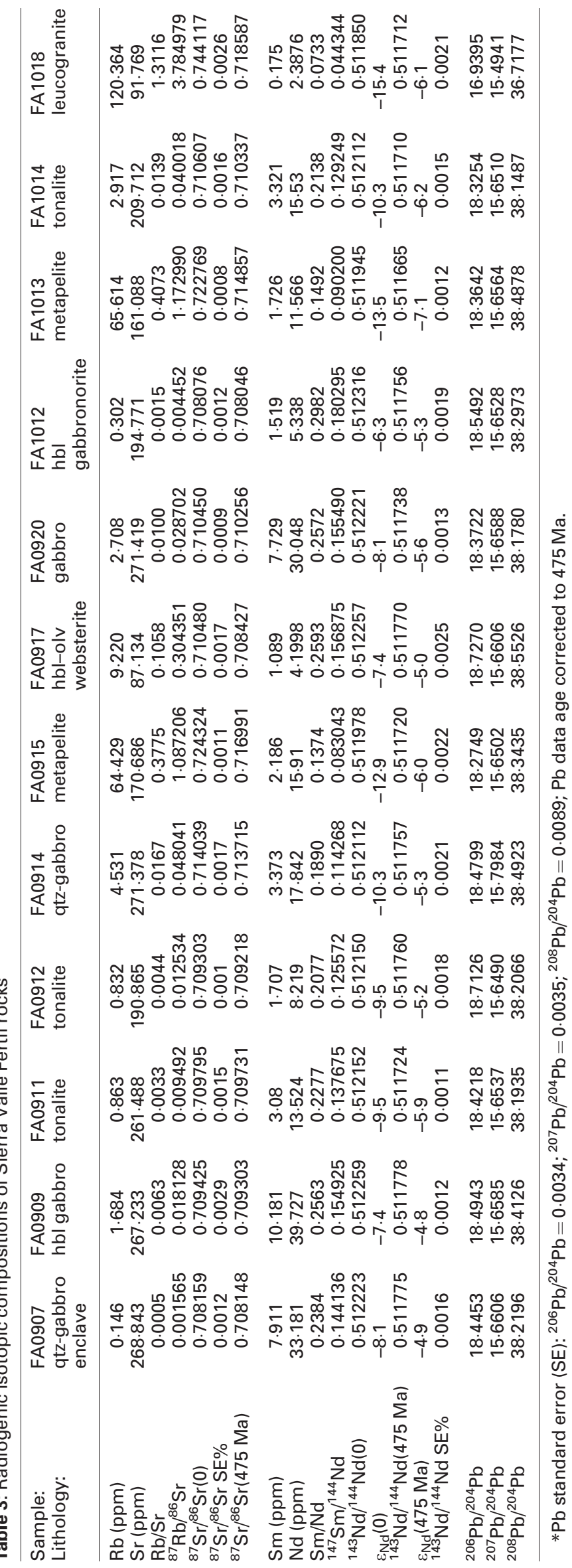



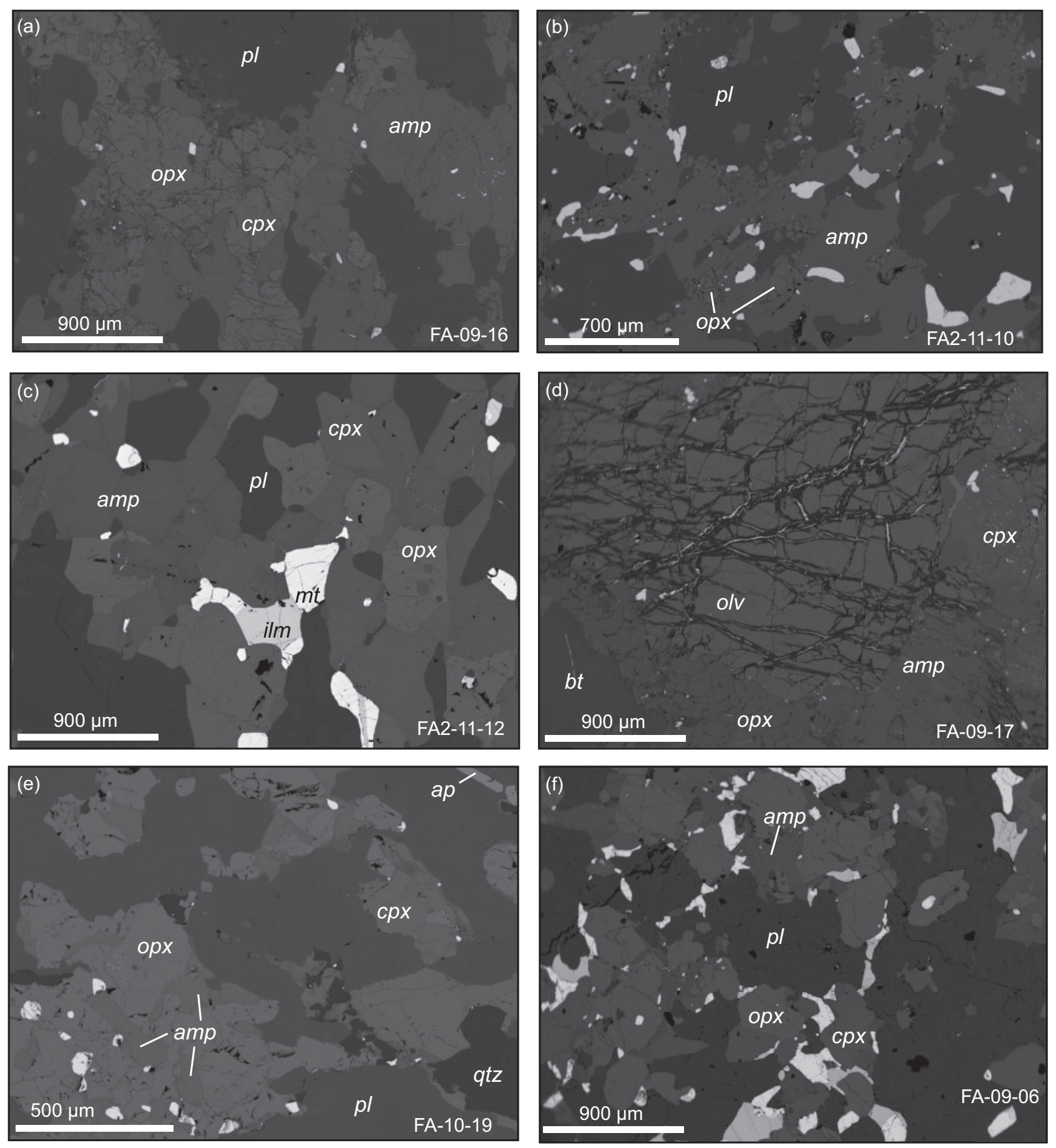

Fig. 10. Back-scattered electron images for the SVF mafic zone rocks, including gabbronorite (a, c, f), gabbro dike (b), amphiboleolivine gabbronorite (d), and tonalite (e). The lack of euhedral grains [with the exception of apatite in (e)] and the total lack of zoning in any mineral should be noted. A consertal texture is common (a, b, e, f), as is a granoblastic texture (c). It should be noted in (d) that the olivine is heavily altered to serpentine, but that fresh domains persist.

There is a significant degree of scatter in the tonalite compositions. This is probably due to additional processes not considered in our simple fractionation model, such as magma mixing and small degrees of assimilation (see Jagoutz, 2010; Putirka et al., 2014). We do not take into account isotopes in our fractionation model (see Otamendi et al., 2012). Additionally, we have not attempted to model the generation of tonalites with $>62 \% \mathrm{SiO}_{2}$. Above $62 \% \mathrm{SiO}_{2}$, a more complex set of assimilation, mixing, and fractionation processes control compositional diversity. For example, $\mathrm{Na}_{2} \mathrm{O}$ decreases with increasing $\mathrm{SiO}_{2}$ beginning at $\sim 62 \% \mathrm{SiO}_{2}$, probably the result of the saturation of an increasingly sodic plagioclase with progressive fractionation, an effect not 
Table 4: Major element compositions of selected olivine from SVF rocks

\begin{tabular}{|c|c|c|c|c|c|c|c|c|}
\hline & FA-12-07 & FA-12-07 & FA-12-07 & FA-12-07 & FA-12-08 & FA-12-08 & FA-12-08 & FA-12-08 \\
\hline $\mathrm{SiO}_{2}$ & 40.02 & 39.49 & $39 \cdot 18$ & $39 \cdot 27$ & $39 \cdot 46$ & 38.95 & 38.5 & $39 \cdot 16$ \\
\hline $\mathrm{Cr}_{2} \mathrm{O}_{3}$ & 0.00 & 0.00 & 0.00 & 0.01 & 0.01 & 0.00 & 0.00 & 0.00 \\
\hline $\mathrm{FeO}$ & $17 \cdot 69$ & $18 \cdot 36$ & $18 \cdot 26$ & $18 \cdot 16$ & $19 \cdot 38$ & $19 \cdot 50$ & $18 \cdot 85$ & $19 \cdot 33$ \\
\hline $\mathrm{MnO}$ & 0.27 & 0.35 & 0.21 & 0.27 & 0.28 & 0.27 & 0.24 & 0.22 \\
\hline $\mathrm{MgO}$ & 41.83 & 41.65 & $42 \cdot 25$ & $42 \cdot 20$ & $40 \cdot 75$ & $41 \cdot 17$ & $42 \cdot 32$ & $41 \cdot 18$ \\
\hline $\mathrm{CaO}$ & 0.01 & 0.00 & 0.00 & 0.00 & 0.01 & 0.01 & 0.02 & 0.01 \\
\hline $\mathrm{NiO}$ & 0.16 & $0 \cdot 15$ & $0 \cdot 10$ & 0.09 & 0.11 & 0.11 & 0.07 & 0.10 \\
\hline Pre-norm total & $99 \cdot 18$ & $99 \cdot 12$ & 99.40 & 99.54 & 99.75 & 99.61 & $100 \cdot 20$ & 99.70 \\
\hline Fo \% & 80.57 & 79.88 & $80 \cdot 30$ & $80 \cdot 32$ & $78 \cdot 70$ & 78.78 & 79.77 & 78.96 \\
\hline$X_{\mathrm{Mg}}$ olivine & 0.81 & 0.80 & 0.80 & 0.80 & 0.79 & 0.79 & 0.80 & 0.79 \\
\hline$X_{\mathrm{Mg}}$ of melt* & 0.55 & 0.54 & 0.55 & 0.55 & 0.53 & 0.53 & 0.54 & 0.53 \\
\hline \multirow[t]{2}{*}{$T$ (liquidus) $\dagger$} & 1249 & 1245 & 1248 & 1248 & 1240 & 1240 & 1245 & 1241 \\
\hline & FA-12-13 & FA-12-13 & FA-12-13 & FA-12-13 & FA-09-17 & FA-09-17 & FA-09-17 & FA-09-17 \\
\hline $\mathrm{SiO}_{2}$ & $39 \cdot 34$ & 39.41 & $39 \cdot 18$ & $39 \cdot 23$ & 38.95 & 38.47 & 38.48 & $38 \cdot 37$ \\
\hline $\mathrm{Cr}_{2} \mathrm{O}_{3}$ & 0.00 & 0.01 & 0.00 & 0.00 & 0.00 & 0.00 & 0.02 & 0.00 \\
\hline $\mathrm{FeO}$ & $17 \cdot 04$ & $16 \cdot 74$ & $17 \cdot 30$ & $17 \cdot 30$ & $20 \cdot 65$ & $21 \cdot 24$ & $20 \cdot 47$ & 21.41 \\
\hline $\mathrm{MnO}$ & 0.26 & $0 \cdot 30$ & 0.28 & 0.28 & 0.28 & 0.29 & 0.28 & 0.28 \\
\hline $\mathrm{MgO}$ & $43 \cdot 16$ & $43 \cdot 29$ & 43.02 & 42.95 & 39.98 & 39.87 & 40.58 & 39.80 \\
\hline $\mathrm{CaO}$ & 0.01 & 0.00 & 0.01 & 0.00 & 0.03 & 0.01 & 0.01 & 0.00 \\
\hline $\mathrm{NiO}$ & 0.20 & 0.25 & 0.21 & 0.22 & 0.12 & $0 \cdot 13$ & $0 \cdot 15$ & 0.13 \\
\hline Pre-norm total & $99 \cdot 11$ & $99 \cdot 75$ & $100 \cdot 27$ & $100 \cdot 31$ & $99 \cdot 12$ & 99.54 & $99 \cdot 32$ & 99.42 \\
\hline Fo \% & 81.63 & 81.90 & $81 \cdot 34$ & $81 \cdot 31$ & $77 \cdot 27$ & $76 \cdot 73$ & $77 \cdot 70$ & 76.58 \\
\hline$X_{\mathrm{Mg}}$ olivine & 0.82 & $0 \cdot 82$ & 0.81 & 0.81 & 0.77 & 0.77 & 0.78 & 0.77 \\
\hline$X_{\mathrm{Mg}}$ of melt & 0.57 & 0.58 & 0.57 & 0.57 & 0.50 & 0.50 & 0.51 & 0.50 \\
\hline$T$ (liquidus)† & 1254 & 1256 & 1253 & 1253 & 1233 & 1231 & 1235 & 1230 \\
\hline
\end{tabular}

${ }^{*} X_{\mathrm{Mg}}$ melt assumes a $K_{\mathrm{D}}{ }^{\text {(ol-liq) }}(\mathrm{Fe}-\mathrm{Mg})=0.32$ (Roeder \& Emslie, 1970; Pichavant \& Macdonald, 2007).

tLiquidus $T$ from Niu et al. (2002).

taken into account by our model. $\mathrm{K}_{2} \mathrm{O}$ remains low $(<1 \mathrm{wt} \%)$ in most mafic zone tonalites, even those with $>73 \% \mathrm{SiO}_{2}$; hence it is likely that these high- $\mathrm{SiO}_{2}$, low$\mathrm{K}$, low-Na tonalites do not represent liquid compositions but have lost melt or fluid rich in $\mathrm{K}$ and $\mathrm{Na}$.

Numerous experiments demonstrate that a range of tonalite compositions can be readily generated via partial melting of a basaltic or amphibolitic source (Beard \& Lofgren, 1989; Rapp, 1991; Wolf \& Wyllie, 1994; Beard, 1995; Rapp \& Watson, 1995; Sisson et al., 2005; Saito et al., 2007). Although the compositions of the SVF mafic zone tonalites are exceptionally low-K and low- $\mathrm{Na}$, they are not altogether dissimilar from tonalites produced experimentally by partial melting. Arc rocks similar in composition to the SVF mafic zone tonalites have been reported elsewhere and interpreted to be the result of partial melting (Beard, 1997; Kawate \& Arima, 1998; Haraguchi et al., 2003; Saito et al., 2004; Smith et al., 2006). However, in the SVF mafic zone we see very little direct evidence supporting a partial melting relationship between the mafic and silicic rocks. We interpret the gabbronorites to be the result of phenocryst accumulation; their modes and compositions display distinctive trends that are not consistent with the residues of partial melting experiments [see fig. 3 of Jagoutz et al., (2011)]. Furthermore, manifest field relations indicating interspersed and intermingled bodies of gabbronorite and tonalite are more consistent with a complex series of intrusive and local melt extraction events. We do not find geological or petrographic evidence that partial melting, as expressed by amphibole breakdown, is a dominant process generating the silicic rocks.

\section{$\mathrm{SiO}_{2}$ enrichment: the importance of amphibole and Fe-Ti oxides}

The process of silica enrichment in a fractionating basaltic magma is problematic because plagioclase and pyroxene-the two most common crystallizing minerals in basalts-have essentially the same composition as the magma. A liquid separated from a plagioclase-pyroxene cumulate would be very similar with respect to its silica content-the so-called 'gabbro barrier' (Davidson et al., 2007; Jagoutz, 2010). For silica enrichment to occur, low-SiO ${ }_{2}$ phases such as olivine, garnet, amphibole, and Fe-Ti oxides must be retained in the cumulate relative to the expelled liquid (Bergantz, 1991; Claeson \& Meurer, 2004; Davidson et al., 2007; Dessimoz et al., 2012; Smith, 2014). Garnet is excluded in our discussion because we find no igneous garnet in the SVF mafic rocks. Olivine is observed only in the ultramafic cumulates. Amphibole and Fe-Ti oxides, however, are abundant in the SVF rocks.

Amphibole is ubiquitous in the Valle Fértil crustal section and forms anywhere from $\sim 5$ to $\sim 40 \%$ of the gabbronorites, although it is rarely found as discrete, sub- to euhedral grains. It is typically present as rims on pyroxenes, and as oikocrysts ranging from $\sim 1$ to $5 \mathrm{~cm}$ in diameter. It is also observed as the dominant mafic 
Table 5: Major element compositions of selected clinopyroxene from SVF rocks

\begin{tabular}{|c|c|c|c|c|c|c|c|c|}
\hline $\begin{array}{l}\text { Sample: } \\
\text { Lithology: }\end{array}$ & $\begin{array}{l}\text { FA2-11-12 } \\
\text { hbl gn }\end{array}$ & $\begin{array}{l}\text { FA2-11-12 } \\
\text { hbl gn }\end{array}$ & $\begin{array}{l}\text { FA2-11-12 } \\
\text { hbl gn }\end{array}$ & $\begin{array}{l}\text { FA2-11-12 } \\
\text { hbl gn }\end{array}$ & $\begin{array}{l}\text { FA-10-19 } \\
\text { tonalite }\end{array}$ & $\begin{array}{l}\text { FA-10-19 } \\
\text { tonalite }\end{array}$ & $\begin{array}{l}\text { FA-10-19 } \\
\text { tonalite }\end{array}$ & $\begin{array}{l}\text { FA-10-19 } \\
\text { tonalite }\end{array}$ \\
\hline $\mathrm{SiO}_{2}$ & $51 \cdot 18$ & $51 \cdot 20$ & $51 \cdot 24$ & $52 \cdot 16$ & $52 \cdot 10$ & 52.06 & 52.77 & $52 \cdot 39$ \\
\hline $\mathrm{TiO}_{2}$ & 0.41 & 0.40 & 0.42 & 0.27 & 0.25 & 0.32 & 0.13 & 0.22 \\
\hline $\mathrm{Al}_{2} \mathrm{O}_{3}$ & 3.04 & 2.99 & $3 \cdot 19$ & $2 \cdot 31$ & $2 \cdot 48$ & $2 \cdot 42$ & 1.48 & 2.25 \\
\hline $\mathrm{Cr}_{2} \mathrm{O}_{3}$ & 0.00 & 0.01 & 0.00 & 0.00 & 0.08 & 0.08 & 0.05 & 0.08 \\
\hline $\mathrm{FeO}$ & 8.54 & $8 \cdot 21$ & 9.53 & 7.64 & 8.92 & $8 \cdot 39$ & $6 \cdot 89$ & 8.00 \\
\hline $\mathrm{MnO}$ & 0.32 & 0.34 & 0.37 & 0.30 & 0.28 & 0.29 & 0.27 & 0.30 \\
\hline $\mathrm{MgO}$ & 13.64 & 13.62 & 13.98 & $14 \cdot 12$ & 13.89 & 13.52 & 14.74 & 13.98 \\
\hline $\mathrm{CaO}$ & 22.51 & $22 \cdot 87$ & 20.93 & 22.90 & 21.62 & 22.44 & $23 \cdot 34$ & $22 \cdot 38$ \\
\hline $\mathrm{Na}_{2} \mathrm{O}$ & 0.37 & 0.36 & 0.34 & 0.30 & 0.38 & 0.49 & 0.32 & 0.40 \\
\hline Total & $100 \cdot 87$ & $100 \cdot 01$ & 99.36 & 99.78 & 99.31 & 99.37 & 99.57 & $100 \cdot 06$ \\
\hline$X_{\mathrm{Mg}} \mathrm{cpx}$ & 0.79 & 0.79 & 0.81 & 0.79 & 0.78 & 0.76 & 0.81 & 0.78 \\
\hline$X_{\mathrm{Mg}}$ melt ${ }^{*}$ & 0.43 & 0.44 & 0.41 & 0.47 & 0.43 & 0.44 & 0.51 & 0.46 \\
\hline Wo & $46 \cdot 74$ & $47 \cdot 42$ & 43.77 & $47 \cdot 20$ & $45 \cdot 13$ & 46.94 & 47.41 & 46.55 \\
\hline En & 39.42 & $39 \cdot 30$ & $40 \cdot 68$ & $40 \cdot 50$ & $40 \cdot 34$ & 39.36 & 41.67 & $40 \cdot 46$ \\
\hline Fs & $13 \cdot 84$ & $13 \cdot 29$ & $15 \cdot 55$ & $12 \cdot 29$ & 14.53 & $13 \cdot 70$ & 10.92 & 12.99 \\
\hline $\begin{array}{l}\text { Sample: } \\
\text { Lithology: }\end{array}$ & $\begin{array}{l}\text { FA2-11-02 } \\
\text { hbl gn }\end{array}$ & $\begin{array}{l}\text { FA2-11-02 } \\
\text { hbl gn }\end{array}$ & $\begin{array}{l}\text { FA-11-02 } \\
\text { hbl gn }\end{array}$ & $\begin{array}{l}\text { FA-11-02 } \\
\text { hbl gn }\end{array}$ & $\begin{array}{l}\text { FA-09-16 } \\
\text { hbl gn }\end{array}$ & $\begin{array}{l}\text { FA-09-16 } \\
\text { hbl gn }\end{array}$ & $\begin{array}{l}\text { FA-09-17 } \\
\text { amph-olv } \\
\text { norite }\end{array}$ & $\begin{array}{l}\text { FA-09-17 } \\
\text { amph-olv } \\
\text { norite }\end{array}$ \\
\hline $\mathrm{SiO}_{2}$ & 51.91 & 51.23 & 51.75 & 52.28 & 52.62 & 51.82 & 51.80 & 51.39 \\
\hline $\mathrm{TiO}_{2}$ & 0.07 & 0.11 & 0.10 & 0.04 & 0.04 & 0.12 & 0.22 & 0.17 \\
\hline $\mathrm{Al}_{2} \mathrm{O}_{3}$ & $2 \cdot 38$ & $2 \cdot 70$ & $2 \cdot 25$ & $2 \cdot 36$ & 1.99 & 2.54 & $3 \cdot 31$ & $4 \cdot 20$ \\
\hline $\mathrm{Cr}_{2} \mathrm{O}_{3}$ & 0.00 & 0.00 & 0.00 & 0.00 & 0.00 & 0.00 & 0.14 & 0.11 \\
\hline $\mathrm{FeO}$ & $9 \cdot 41$ & $10 \cdot 82$ & 9.96 & $8 \cdot 80$ & $7 \cdot 76$ & 8.22 & 4.78 & 5.59 \\
\hline $\mathrm{MnO}$ & 0.38 & 0.43 & 0.40 & 0.33 & 0.20 & 0.20 & $0 \cdot 12$ & 0.20 \\
\hline $\mathrm{MgO}$ & $13 \cdot 31$ & $13 \cdot 60$ & $13 \cdot 29$ & 13.55 & $14 \cdot 20$ & 14.03 & $15 \cdot 61$ & 14.98 \\
\hline $\mathrm{CaO}$ & 22.06 & $20 \cdot 60$ & 21.76 & $22 \cdot 18$ & $22 \cdot 81$ & 22.63 & 23.79 & 23.07 \\
\hline $\mathrm{Na}_{2} \mathrm{O}$ & 0.48 & 0.51 & 0.49 & 0.46 & 0.38 & 0.46 & 0.23 & 0.29 \\
\hline Total & 99.79 & 99.17 & $100 \cdot 12$ & 99.70 & $100 \cdot 41$ & $100 \cdot 01$ & 99.56 & 99.36 \\
\hline$X_{\mathrm{Mg}} \mathrm{cpx}$ & 0.76 & 0.79 & 0.76 & 0.76 & 0.79 & 0.80 & 0.89 & 0.87 \\
\hline$X_{\mathrm{Mg}}$ melt ${ }^{*}$ & 0.41 & 0.38 & 0.39 & 0.43 & 0.47 & 0.45 & 0.61 & 0.56 \\
\hline Wo & 46.03 & 42.94 & $45 \cdot 31$ & $46 \cdot 30$ & 46.91 & 46.60 & 48.31 & 47.79 \\
\hline En & 38.64 & 39.45 & 38.50 & $39 \cdot 36$ & 40.63 & $40 \cdot 19$ & 44.11 & $43 \cdot 18$ \\
\hline Fs & $15 \cdot 33$ & 17.61 & $16 \cdot 19$ & 14.34 & 12.46 & $13 \cdot 21$ & $7 \cdot 58$ & 9.04 \\
\hline
\end{tabular}

${ }^{*} X_{\mathrm{Mg}}$ (melt) assumes a $K_{\mathrm{D}}{ }^{(\mathrm{cpx}-\mathrm{liq})}=0.27$ (Sisson \& Grove, 1993; Pichavant \& McDonald, 2007).

mineral in gabbroic dikes and in a few relict troctolites. Texturally, then, it appears that amphibole either replaces other minerals or was a late phase on the liquidus of the Valle Fértil magmas. Nevertheless, other compositional trends, such as depleted $\mathrm{Zr} / \mathrm{Hf}$ in amphibole gabbronorites and steepening REE trends with increasing $\mathrm{SiO}_{2}$ [although $\mathrm{Dy} / \mathrm{Yb}$ does not change appreciably (see Davidson et al., 2007)] indicate that amphibole crystallization exerted an influence on the fractionating magma compositions, like that commonly reported in xenoliths from arcs (Smith, 2014).

In addition to amphibole, Fe-Ti oxide fractionation produces silica enrichment. In the SVF, magnetite and ilmenite appear in all gabbronorites. The cumulate subgroups, as identified on the $X_{\mathrm{Mg}}$ vs $\mathrm{SiO}_{2}$ plot, have notable differences in $\mathrm{Fe}-\mathrm{Ti}$ oxide abundances. Fe-Ti oxides increase in abundance with progression along the 'cumulate line of descent'. High- $X_{\mathrm{Mg}}$ rocks ( $>\sim 0.6$; red and orange cumulate groups in Fig. 13) have trace amounts of $\mathrm{Fe}-\mathrm{Ti}$ oxides. Intermediate- $X_{\mathrm{Mg}}$ rocks $(0.5-$ 0.6; green cumulate group) have appreciable $\mathrm{Fe}-\mathrm{Ti}$ oxides ( $<\sim 3 \%$ magnetite, $<\sim 1.2 \%$ ilmenite). Cumulates with the lowest $X_{\mathrm{Mg}}(\sim 0.4-0.5$; blue cumulate group) and the lowest $\mathrm{SiO}_{2}(\sim 41-45 \%)$ are particularly rich in magnetite and ilmenite $(\sim 4 \%$ and $\sim 1-2 \%$ respectively, with consequently high concentrations of $\mathrm{TiO}_{2}$ and $\mathrm{FeO}$; Fig. 14; see also Fig. 6). It is notable that there is no such relationship between amphibole mode and whole-rock composition. Hence it is likely that the accumulation of Fe-Ti oxides is perhaps more important than amphibole in producing silica enrichment in some arc rocks. The extreme abundances of $\mathrm{Fe}-\mathrm{Ti}$ oxides in certain cumulate lithologies suggest that, in the fractionation sequence, $\mathrm{Fe}-\mathrm{Ti}$ oxide 'blooms' may occur, perhaps during recharge of mafic magmas or when the thermodynamic or magmatic state favors increased crystallization and accumulation.

\section{Assimilation of country rock}

The Famatinian arc was constructed on, and within, a fertile, miogeoclinal sequence on the edge of the protoSouth American continent (Otamendi et al., 2008; Cristofolini et al., 2012). It therefore should not be viewed as an 'Andean' arc because it was not emplaced within an old, thick craton (Ducea et al., 2010). Neither should it be viewed as an island arc, because it was constructed on the edge of a continent. Petrogenetic 
Table 6: Major element compositions of selected orthopyroxene from SVF rocks

\begin{tabular}{|c|c|c|c|c|c|c|c|c|}
\hline $\begin{array}{l}\text { Sample: } \\
\text { Lithology: }\end{array}$ & $\begin{array}{l}\text { FA2-11-12 } \\
\text { hbl gn }\end{array}$ & $\begin{array}{l}\text { FA2-11-12 } \\
\text { hbl gn }\end{array}$ & $\begin{array}{l}\text { FA2-11-12 } \\
\text { hbl gn }\end{array}$ & $\begin{array}{l}\text { FA2-11-12 } \\
\text { hbl gn }\end{array}$ & $\begin{array}{l}\text { FA-10-19 } \\
\text { tonalite }\end{array}$ & $\begin{array}{l}\text { FA-10-19 } \\
\text { tonalite }\end{array}$ & $\begin{array}{l}\text { FA-10-05 } \\
\text { tonalite }\end{array}$ & $\begin{array}{l}\text { FA-10-05 } \\
\text { tonalite }\end{array}$ \\
\hline $\mathrm{SiO}_{2}$ & 52.52 & 52.56 & $52 \cdot 41$ & $52 \cdot 86$ & 52.91 & $52 \cdot 46$ & 51.87 & $52 \cdot 14$ \\
\hline $\mathrm{TiO}_{2}$ & 0.13 & 0.08 & 0.08 & 0.08 & 0.08 & 0.09 & 0.09 & 0.08 \\
\hline $\mathrm{Al}_{2} \mathrm{O}_{3}$ & 1.52 & 1.37 & 1.30 & $1 \cdot 16$ & $1 \cdot 14$ & 1.01 & 1.44 & 1.36 \\
\hline $\mathrm{Cr}_{2} \mathrm{O}_{3}$ & 0.02 & 0.02 & 0.01 & 0.01 & 0.03 & 0.03 & 0.00 & 0.01 \\
\hline $\mathrm{MnO}$ & 0.89 & 0.80 & 0.77 & 0.85 & 0.66 & 0.86 & $1 \cdot 13$ & $1 \cdot 18$ \\
\hline $\mathrm{MgO}$ & 22.77 & $22 \cdot 60$ & 22.79 & 22.69 & 22.44 & 21.48 & 21.02 & 21.34 \\
\hline $\mathrm{FeO}$ & 21.62 & $22 \cdot 25$ & $22 \cdot 19$ & 21.90 & $22 \cdot 30$ & 23.64 & 23.90 & $23 \cdot 37$ \\
\hline $\mathrm{CaO}$ & 0.53 & 0.32 & 0.45 & 0.45 & 0.44 & 0.42 & 0.54 & 0.52 \\
\hline $\mathrm{Na}_{2} \mathrm{O}$ & 0.01 & 0.00 & 0.00 & 0.00 & 0.00 & 0.00 & 0.00 & 0.00 \\
\hline Total* & 99.62 & 99.59 & 99.44 & $99 \cdot 28$ & 99.03 & 99.06 & 99.57 & 98.95 \\
\hline$X_{\mathrm{Mg}}$ & 0.65 & 0.64 & 0.65 & 0.65 & 0.64 & 0.62 & 0.61 & 0.62 \\
\hline En & 64.54 & 64.00 & 64.09 & $64 \cdot 28$ & 63.64 & $61 \cdot 30$ & $60 \cdot 38$ & $61 \cdot 28$ \\
\hline Fs & $34 \cdot 37$ & $35 \cdot 34$ & 35.00 & $34 \cdot 80$ & 35.47 & 37.84 & 38.51 & 37.64 \\
\hline Wo & 1.09 & 0.66 & 0.92 & 0.92 & 0.89 & 0.86 & $1 \cdot 12$ & 1.07 \\
\hline $\begin{array}{l}\text { Sample: } \\
\text { Lithology: }\end{array}$ & $\begin{array}{l}\text { FA2-11-02 } \\
\text { hbl gn }\end{array}$ & $\begin{array}{l}\text { FA2-11-02 } \\
\text { hbl gn }\end{array}$ & $\begin{array}{l}\text { FA-09-16 } \\
\text { hbl gn }\end{array}$ & $\begin{array}{l}\text { FA-09-16 } \\
\text { hbl gn }\end{array}$ & $\begin{array}{l}\text { FA-09-17 } \\
\text { amph-olv } \\
\text { norite }\end{array}$ & $\begin{array}{l}\text { FA-09-17 } \\
\text { amph-olv } \\
\text { norite }\end{array}$ & $\begin{array}{l}\text { FA-09-20 } \\
\text { norite }\end{array}$ & $\begin{array}{l}\text { FA-09-20 } \\
\text { norite }\end{array}$ \\
\hline $\mathrm{SiO}_{2}$ & 51.83 & $51 \cdot 18$ & 52.60 & $52 \cdot 86$ & 53.88 & $53 \cdot 18$ & 51.76 & $52 \cdot 16$ \\
\hline $\mathrm{TiO}_{2}$ & 0.00 & 0.00 & 0.00 & 0.00 & 0.02 & 0.07 & 0.12 & 0.09 \\
\hline $\mathrm{Al}_{2} \mathrm{O}_{3}$ & 1.54 & 1.63 & 1.41 & 1.37 & $3 \cdot 14$ & $3 \cdot 36$ & 1.26 & $1 \cdot 17$ \\
\hline $\mathrm{Cr}_{2} \mathrm{O}_{3}$ & 0.00 & 0.00 & 0.00 & 0.00 & 0.09 & 0.07 & 0.00 & 0.00 \\
\hline $\mathrm{MnO}$ & 0.83 & 0.90 & 0.59 & 0.51 & 0.31 & 0.30 & 1.13 & 1.06 \\
\hline $\mathrm{MgO}$ & $21 \cdot 17$ & $20 \cdot 44$ & 22.63 & $22 \cdot 64$ & 28.48 & 28.56 & $20 \cdot 81$ & 21.03 \\
\hline $\mathrm{FeO}$ & $24 \cdot 20$ & $25 \cdot 44$ & $22 \cdot 36$ & $22 \cdot 23$ & $13 \cdot 74$ & 14.00 & 23.96 & 24.03 \\
\hline $\mathrm{CaO}$ & 0.42 & 0.39 & 0.40 & 0.37 & 0.32 & 0.45 & 0.94 & 0.46 \\
\hline $\mathrm{Na}_{2} \mathrm{O}$ & 0.01 & 0.02 & 0.01 & 0.02 & 0.00 & 0.00 & 0.00 & 0.00 \\
\hline Total* & 99.78 & 99.91 & 99.80 & $100 \cdot 47$ & 99.80 & 99.86 & $99 \cdot 32$ & 99.94 \\
\hline$X_{\mathrm{Mg}}$ & 0.61 & 0.59 & 0.64 & 0.64 & 0.79 & 0.78 & 0.61 & 0.61 \\
\hline En & $60 \cdot 41$ & 58.41 & 63.82 & 64.00 & $78 \cdot 20$ & 77.74 & 59.58 & $60 \cdot 36$ \\
\hline Fs & 38.74 & $40 \cdot 78$ & 35.37 & $35 \cdot 25$ & $21 \cdot 16$ & 21.38 & 38.48 & 38.69 \\
\hline Wo & 0.86 & 0.81 & 0.81 & 0.74 & 0.64 & 0.88 & 1.94 & 0.95 \\
\hline
\end{tabular}

*Pre-normalization totals.

gn, gabbronorite.

studies based on isotope data from the Kohistan (Petterson et al., 1993; Dhuime et al., 2009; Jagoutz, 2010) and Talkeetna (Rioux et al., 2007) crustal sections-both paleo island arcs-suggest that assimilation of pre-existing country rocks plays only a minor role in contributing to the mass of arc magmas. Some studies on the Bonanza arc have made similar arguments (Cui \& Russell, 1995; Larocque \& Canil, 2010), although there are differing opinions (see DeBari et al., 1999). The Kohistan and Talkeetna arcs intrude preexisting crust that is dominantly oceanic in composition, with isotopic characteristics similar to those of typical mantle. However, in the western domain of the Talkeetna complex, isotopic compositions are more variable and are interpreted to reflect the influence of the adjacent Wrangellia terrane (Rioux et al., 2007). Some Bonanza arc rocks show isotopic influence from their country rocks (older arc rocks along with some clastic sediments and carbonates), but many of the country rocks themselves are primitive isotopically (Samson et al., 1990; Andrew et al., 1991). A possible analogue to the SVF is the Salinian block (California, USA), where late Cretaceous granitoids intrude a thick sequence of sedimentary rock (Ducea et al., 2003; Kidder et al., 2003).
In the upper portions of the SVF arc section, which is dominated by tonalite and granodiorite, field and compositional evidence for the assimilation of country-rock by the SVF magmas is unequivocal (Otamendi et al., 2009a, 2010b, 2012). In the mafic complex, sparse field relations clearly exhibit the process of country-rock melting and reaction (Otamendi et al., 2008; Gallien et al., 2010; Cristofolini et al., 2012). Additionally, Ducea et al. (2010) reported the presence of xenocrystic zircons from one sample from the mafic zone. Finally, the radiogenic isotope compositions of the SVF rocks have a crustal component, with initial $(475 \mathrm{Ma}){ }^{87} \mathrm{Sr} /{ }^{86} \mathrm{Sr}>$ 0.7052 and $\varepsilon_{\mathrm{Nd}}<2.1$, with most rocks having $\mathrm{Sr}_{\mathrm{i}}>0.708$ and $\varepsilon_{\text {Nd }}<-4.0$ (Fig. 9; Otamendi et al., 2012). Even the gabbronorites of the mafic zone have crustal isotopic signatures, although many are deficient in $\mathrm{SiO}_{2}(<47 \%)$ and $\mathrm{K}_{2} \mathrm{O}(<0.5 \%)$. This crustal isotopic affinity of the SVF rocks can plausibly be attributed in part to a 'polluted' mantle source in which the lithospheric mantle was already contaminated by crustal material, whether by lower crustal delamination, subduction erosion, or some other mechanism (Stern, 1991; Kay \& Mahlburg Kay, 1993; Ducea \& Saleeby, 1998; Lackey et al., 2005). However, even with a contaminated mantle source some amount of crust is necessary to explain the 
Table 7: Compositions of select amphibole grains from SVF rocks

\begin{tabular}{|c|c|c|c|c|c|c|c|c|}
\hline $\begin{array}{l}\text { Sample: } \\
\text { Lithology: }\end{array}$ & $\begin{array}{l}\text { FA2-11- } \\
02 \\
\text { hbl gn }\end{array}$ & $\begin{array}{l}\text { FA2-11- } \\
02 \\
\text { hbl gn }\end{array}$ & $\begin{array}{l}\text { FA2-11- } \\
02 \\
\text { hbl gn }\end{array}$ & $\begin{array}{l}\text { FA2-11- } \\
02 \\
\text { hbl gn }\end{array}$ & $\begin{array}{l}\text { FA2-11- } \\
10 \\
\text { hbl gn }\end{array}$ & $\begin{array}{l}\text { FA2-11- } \\
10 \\
\text { hbl gn }\end{array}$ & $\begin{array}{l}\text { FA2-11- } \\
10 \\
\text { hbl gn }\end{array}$ & $\begin{array}{l}\text { FA2-11- } \\
10 \\
\text { hbl gn }\end{array}$ \\
\hline $\mathrm{SiO}_{2}$ & 43.35 & 44.74 & 42.75 & 43.83 & 44.94 & 42.65 & 43.15 & 44.46 \\
\hline $\mathrm{TiO}_{2}$ & 1.46 & 1.54 & 1.70 & 1.78 & 1.53 & $2 \cdot 18$ & 1.91 & 1.59 \\
\hline $\mathrm{Al}_{2} \mathrm{O}_{3}$ & $11 \cdot 13$ & 9.37 & 11.47 & $10 \cdot 76$ & $10 \cdot 36$ & 11.73 & 11.74 & $11 \cdot 19$ \\
\hline $\mathrm{Cr}_{2} \mathrm{O}_{3}$ & 0.04 & 0.02 & 0.02 & 0.00 & 0.02 & 0.04 & 0.02 & 0.00 \\
\hline $\mathrm{FeO}$ & 14.16 & 14.90 & 14.54 & 14.21 & 11.60 & 12.60 & 12.22 & 11.13 \\
\hline $\mathrm{MnO}$ & 0.18 & 0.27 & 0.16 & 0.21 & 0.16 & 0.14 & 0.13 & 0.12 \\
\hline $\mathrm{MgO}$ & 12.49 & 12.45 & $12 \cdot 36$ & 12.67 & 14.63 & $13 \cdot 28$ & $13 \cdot 33$ & 14.26 \\
\hline $\mathrm{CaO}$ & 11.62 & 11.48 & $11 \cdot 19$ & 11.78 & 11.76 & 11.80 & 11.96 & 12.07 \\
\hline $\mathrm{Na}_{2} \mathrm{O}$ & 1.37 & 1.27 & 0.98 & 1.34 & 1.56 & 1.72 & 1.69 & 1.52 \\
\hline $\mathrm{K}_{2} \mathrm{O}$ & 1.37 & 1.09 & 1.04 & 1.28 & 1.10 & 1.36 & 1.33 & 1.31 \\
\hline Total & $97 \cdot 17$ & 97.13 & 96.21 & 97.86 & 97.66 & 97.51 & 97.48 & 97.65 \\
\hline $\mathrm{Si}$ & 6.39 & $6 \cdot 59$ & $6 \cdot 30$ & 6.42 & 6.49 & 6.26 & 6.33 & $6 \cdot 46$ \\
\hline $\mathrm{Al}_{\text {iv }}$ & 1.61 & 1.41 & 1.70 & 1.58 & 1.51 & 1.74 & 1.67 & 1.54 \\
\hline $\mathrm{Al}_{\mathrm{vi}}$ & 0.32 & 0.22 & 0.29 & 0.27 & 0.26 & 0.29 & 0.36 & 0.37 \\
\hline $\mathrm{Ti}$ & 0.16 & 0.17 & 0.19 & 0.20 & 0.17 & 0.24 & 0.21 & 0.17 \\
\hline $\mathrm{Cr}$ & 0.00 & 0.00 & 0.00 & 0.00 & 0.00 & 0.00 & 0.00 & 0.00 \\
\hline $\mathrm{Fe}^{3+}$ & 0.64 & 0.65 & 1.04 & 0.60 & 0.63 & 0.51 & 0.40 & 0.40 \\
\hline $\mathrm{Fe}^{2+}$ & 1.10 & 1.19 & 0.75 & 1.14 & 0.77 & 1.03 & 1.10 & 0.95 \\
\hline $\mathrm{Mn}$ & 0.02 & 0.03 & 0.02 & 0.03 & 0.02 & 0.02 & 0.02 & 0.01 \\
\hline $\mathrm{Mg}$ & 2.74 & 2.74 & $2 \cdot 71$ & 2.77 & 3.15 & 2.90 & 2.91 & 3.09 \\
\hline $\mathrm{Ca}$ & 1.83 & 1.81 & 1.77 & 1.85 & 1.82 & 1.85 & 1.88 & 1.88 \\
\hline $\mathrm{Na}$ & 0.39 & 0.36 & 0.28 & 0.38 & 0.44 & 0.49 & 0.48 & 0.43 \\
\hline K & 0.26 & 0.21 & 0.20 & 0.24 & 0.20 & 0.26 & 0.25 & 0.24 \\
\hline Total & 17.48 & 17.38 & 17.24 & 17.47 & 17.46 & 17.60 & 17.61 & 17.55 \\
\hline $\mathrm{Na}(\mathrm{B})$ & 0.17 & $0 \cdot 19$ & 0.23 & 0.15 & 0.18 & 0.15 & 0.12 & 0.12 \\
\hline $\mathrm{Ca}(\mathrm{B})$ & 1.83 & 1.81 & 1.77 & 1.85 & 1.82 & 1.85 & 1.88 & 1.88 \\
\hline$(\mathrm{Na}+\mathrm{K})(\mathrm{A})$ & 0.48 & 0.38 & 0.24 & 0.47 & 0.46 & 0.60 & 0.61 & 0.55 \\
\hline $\mathrm{Mg} /\left(\mathrm{Mg}+\mathrm{Fe}^{2+}\right)$ & 0.71 & 0.70 & 0.78 & 0.71 & 0.80 & 0.74 & 0.73 & 0.76 \\
\hline Mg\# (total Fe) & 0.61 & 0.60 & 0.60 & 0.61 & 0.69 & 0.65 & 0.66 & 0.70 \\
\hline $\mathrm{Fe}^{3+} /\left(\mathrm{Fe}^{3+}+\mathrm{Al}_{\mathrm{vi}}\right)$ & 0.67 & 0.74 & 0.78 & 0.69 & 0.71 & 0.64 & 0.53 & 0.52 \\
\hline $\begin{array}{l}\text { Sample: } \\
\text { Lithology: }\end{array}$ & $\begin{array}{l}\text { FA-09- } \\
16 \\
\text { hbl gn }\end{array}$ & $\begin{array}{l}\text { FA-09- } \\
16 \\
\text { hbl an }\end{array}$ & $\begin{array}{l}\text { FA-09- } \\
16 \\
\text { hbl gn }\end{array}$ & $\begin{array}{l}\text { FA-09- } \\
16 \\
\text { hbl gn }\end{array}$ & $\begin{array}{l}\text { FA-10- } \\
19 \\
\text { tonalite }\end{array}$ & $\begin{array}{l}\text { FA-10- } \\
19 \\
\text { tonalite }\end{array}$ & $\begin{array}{l}\text { FA-10- } \\
19 \\
\text { tonalite }\end{array}$ & $\begin{array}{l}\text { FA-10- } \\
19 \\
\text { tonalite }\end{array}$ \\
\hline $\mathrm{SiO}_{2}$ & $45 \cdot 25$ & $45 \cdot 18$ & 45.66 & $45 \cdot 34$ & 44.90 & $45 \cdot 16$ & 44.39 & $45 \cdot 10$ \\
\hline $\mathrm{TiO}_{2}$ & 1.44 & 1.59 & 1.48 & 1.52 & 1.43 & 1.09 & 1.76 & 1.57 \\
\hline $\mathrm{Al}_{2} \mathrm{O}_{3}$ & 9.80 & $10 \cdot 09$ & 9.55 & 9.61 & 10.56 & 10.53 & 10.56 & $10 \cdot 10$ \\
\hline $\mathrm{Cr}_{2} \mathrm{O}_{3}$ & $0 \cdot 10$ & 0.17 & $0 \cdot 14$ & 0.11 & 0.08 & 0.15 & 0.23 & 0.10 \\
\hline $\mathrm{FeO}$ & 12.79 & $12 \cdot 23$ & 11.92 & 12.03 & 12.93 & $12 \cdot 42$ & 12.92 & 12.57 \\
\hline $\mathrm{MnO}$ & 0.17 & 0.10 & 0.12 & 0.09 & 0.16 & 0.11 & 0.17 & 0.14 \\
\hline $\mathrm{MgO}$ & 13.92 & $14 \cdot 11$ & 14.38 & 14.26 & 13.43 & 14.02 & 13.28 & 13.61 \\
\hline $\mathrm{CaO}$ & 11.98 & 11.93 & 12.08 & 12.03 & 11.78 & 11.65 & 11.71 & 11.76 \\
\hline $\mathrm{Na}_{2} \mathrm{O}$ & 1.33 & 1.36 & 1.26 & 1.38 & 0.97 & 0.99 & 1.09 & 0.94 \\
\hline $\mathrm{K}_{2} \mathrm{O}$ & 0.72 & 0.78 & 0.73 & 0.72 & 0.95 & 0.95 & 1.14 & 1.01 \\
\hline Total & 97.51 & 97.52 & $97 \cdot 31$ & $97 \cdot 10$ & $97 \cdot 19$ & 97.08 & 97.25 & 96.90 \\
\hline Si & 6.57 & $6 \cdot 54$ & $6 \cdot 62$ & $6 \cdot 60$ & $6 \cdot 52$ & 6.53 & 6.48 & 6.57 \\
\hline $\mathrm{Al}_{\text {iv }}$ & 1.43 & 1.46 & 1.38 & 1.40 & 1.48 & 1.47 & 1.52 & 1.43 \\
\hline $\mathrm{Al}_{\mathrm{vi}}$ & 0.24 & 0.27 & 0.25 & 0.25 & 0.33 & 0.33 & 0.29 & 0.31 \\
\hline $\mathrm{Ti}$ & $0 \cdot 16$ & 0.17 & 0.16 & 0.17 & 0.16 & 0.12 & 0.19 & 0.17 \\
\hline $\mathrm{Cr}$ & 0.01 & 0.02 & 0.02 & 0.01 & 0.01 & 0.02 & 0.03 & 0.01 \\
\hline $\mathrm{Fe}^{3+}$ & 0.63 & 0.59 & 0.54 & 0.53 & 0.70 & 0.82 & 0.63 & 0.64 \\
\hline $\mathrm{Fe}^{2+}$ & 0.93 & 0.89 & 0.90 & 0.94 & 0.87 & 0.68 & 0.94 & 0.90 \\
\hline $\mathrm{Mn}$ & 0.02 & 0.01 & 0.01 & 0.01 & 0.02 & 0.01 & 0.02 & 0.02 \\
\hline $\mathrm{Mg}$ & 3.01 & 3.05 & $3 \cdot 11$ & 3.09 & 2.91 & 3.02 & 2.89 & 2.96 \\
\hline $\mathrm{Ca}$ & 1.86 & 1.85 & 1.88 & 1.88 & 1.83 & 1.81 & 1.83 & 1.84 \\
\hline $\mathrm{Na}$ & 0.37 & 0.38 & 0.35 & 0.39 & 0.27 & 0.28 & 0.31 & 0.26 \\
\hline $\mathrm{K}$ & 0.13 & 0.14 & 0.13 & 0.13 & 0.18 & $0 \cdot 18$ & 0.21 & 0.19 \\
\hline Total & $17 \cdot 37$ & $17 \cdot 38$ & $17 \cdot 36$ & $17 \cdot 40$ & $17 \cdot 28$ & $17 \cdot 26$ & $17 \cdot 35$ & $17 \cdot 29$ \\
\hline $\mathrm{Na}(\mathrm{B})$ & 0.14 & 0.15 & 0.12 & 0.12 & 0.17 & 0.19 & 0.17 & 0.16 \\
\hline $\mathrm{Ca}(\mathrm{B})$ & 1.86 & 1.85 & 1.88 & 1.88 & 1.83 & 1.81 & 1.83 & 1.84 \\
\hline$(\mathrm{Na}+\mathrm{K})(\mathrm{A})$ & 0.37 & 0.38 & 0.36 & 0.40 & 0.28 & 0.26 & 0.35 & 0.29 \\
\hline $\mathrm{Mg} /\left(\mathrm{Mg}+\mathrm{Fe}^{2+}\right)$ & 0.76 & 0.77 & 0.78 & 0.77 & 0.77 & 0.82 & 0.75 & 0.77 \\
\hline Mg\# (total Fe) & 0.66 & 0.67 & 0.68 & 0.68 & 0.65 & 0.67 & 0.65 & 0.66 \\
\hline $\mathrm{Fe}^{3+} /\left(\mathrm{Fe}^{3+}+\mathrm{Al}_{\mathrm{vi}}\right)$ & 0.72 & 0.69 & 0.68 & 0.68 & 0.68 & 0.72 & 0.68 & 0.67 \\
\hline
\end{tabular}

Structural formula determined using cation charge summing to 46 , and $\mathrm{Fe}^{2+} / \mathrm{Fe}^{3+}$ estimated on the basis of 13 cations, after Leake et al. (1997). 
Table 8: Compositions of selected plagioclase from SVF rocks

\begin{tabular}{|c|c|c|c|c|c|c|c|c|}
\hline $\begin{array}{l}\text { Sample: } \\
\text { Lithology: }\end{array}$ & $\begin{array}{l}\text { FA-10-05 } \\
\text { tonalite }\end{array}$ & $\begin{array}{l}\text { FA-10-05 } \\
\text { tonalite }\end{array}$ & $\begin{array}{l}\text { FA-10-05 } \\
\text { tonalite }\end{array}$ & $\begin{array}{l}\text { FA-11-12 } \\
\text { tonalite }\end{array}$ & $\begin{array}{l}\text { FA-11-12 } \\
\text { tonalite }\end{array}$ & $\begin{array}{l}\text { FA-11-12 } \\
\text { tonalite }\end{array}$ & $\begin{array}{l}\text { FA2-11-10 } \\
\text { hbl gn }\end{array}$ & $\begin{array}{l}\text { FA2-11-10 } \\
\text { hbl gn }\end{array}$ \\
\hline $\mathrm{SiO}_{2}$ & 54.97 & 55.66 & 56.50 & $55 \cdot 70$ & 54.52 & 52.64 & $46 \cdot 19$ & $45 \cdot 71$ \\
\hline $\mathrm{Al}_{2} \mathrm{O}_{3}$ & $28 \cdot 70$ & 27.99 & 27.68 & 27.90 & 28.55 & 29.95 & 34.59 & 34.37 \\
\hline $\mathrm{FeO}$ & 0.05 & 0.11 & 0.18 & 0.02 & 0.09 & 0.08 & 0.14 & 0.89 \\
\hline $\mathrm{CaO}$ & 10.87 & $10 \cdot 38$ & $9 \cdot 46$ & $10 \cdot 36$ & $11 \cdot 34$ & 12.71 & $17 \cdot 50$ & 17.68 \\
\hline $\mathrm{SrO}$ & 0.01 & 0.08 & 0.05 & 0.08 & 0.08 & 0.08 & 0.04 & 0.03 \\
\hline $\mathrm{Na}_{2} \mathrm{O}$ & $5 \cdot 24$ & $5 \cdot 45$ & 5.69 & $5 \cdot 82$ & $5 \cdot 31$ & $4 \cdot 47$ & 1.50 & 1.26 \\
\hline $\mathrm{K}_{2} \mathrm{O}$ & 0.16 & 0.32 & 0.44 & 0.12 & 0.11 & 0.07 & 0.04 & 0.07 \\
\hline Total* & 99.94 & $100 \cdot 48$ & $100 \cdot 62$ & 99.70 & $100 \cdot 22$ & $100 \cdot 11$ & 100.56 & 99.69 \\
\hline$X_{\mathrm{An}}$ & 0.78 & 0.76 & 0.73 & 0.76 & 0.79 & 0.83 & 0.95 & 0.96 \\
\hline$X_{\mathrm{Ab}}$ & 0.21 & 0.22 & 0.24 & 0.24 & 0.20 & 0.16 & 0.05 & 0.04 \\
\hline $\begin{array}{l}\text { Sample: } \\
\text { Lithology: }\end{array}$ & $\begin{array}{l}\text { FA-09-17 } \\
\text { amph-olv } \\
\text { norite }\end{array}$ & $\begin{array}{l}\text { FA-09-17 } \\
\text { amph-olv } \\
\text { norite }\end{array}$ & $\begin{array}{l}\text { FA2-11-02 } \\
\text { hbl gn }\end{array}$ & $\begin{array}{l}\text { FA2-11-02 } \\
\text { hbl gn }\end{array}$ & $\begin{array}{l}\text { FA-09-16 } \\
\text { hbl gn }\end{array}$ & $\begin{array}{l}\text { FA-09-16 } \\
\text { hbl gn }\end{array}$ & $\begin{array}{l}\text { FA-09-06 } \\
\text { hbl gn }\end{array}$ & $\begin{array}{l}\text { FA-09-06 } \\
\text { hbl gn }\end{array}$ \\
\hline $\mathrm{SiO}_{2}$ & 43.83 & 44.67 & $50 \cdot 28$ & $53 \cdot 19$ & 46.68 & $45 \cdot 38$ & $45 \cdot 19$ & 45.63 \\
\hline $\mathrm{Al}_{2} \mathrm{O}_{3}$ & 35.74 & $35 \cdot 19$ & 31.73 & 29.52 & $34 \cdot 10$ & 34.93 & 34.61 & 34.43 \\
\hline $\mathrm{FeO}$ & 0.10 & 0.11 & 0.20 & 0.13 & 0.21 & 0.17 & 0.21 & 0.59 \\
\hline $\mathrm{CaO}$ & $19 \cdot 47$ & 18.78 & $14 \cdot 34$ & $12 \cdot 29$ & $17 \cdot 22$ & $18 \cdot 21$ & 18.46 & $17 \cdot 75$ \\
\hline $\mathrm{SrO}$ & 0.06 & 0.08 & 0.02 & 0.06 & 0.05 & 0.05 & 0.03 & 0.07 \\
\hline $\mathrm{Na}_{2} \mathrm{O}$ & 0.79 & $1 \cdot 14$ & 3.29 & $4 \cdot 61$ & 1.70 & 1.23 & 1.48 & 1.51 \\
\hline $\mathrm{K} 2 \mathrm{O}$ & 0.02 & 0.04 & 0.13 & 0.21 & 0.04 & 0.03 & 0.02 & 0.03 \\
\hline Total* & 99.93 & 99.95 & 99.98 & $100 \cdot 16$ & $100 \cdot 41$ & $100 \cdot 14$ & 99.84 & 99.68 \\
\hline$X_{\text {An }}$ & 0.98 & 0.97 & 0.88 & 0.82 & 0.95 & 0.96 & 0.96 & 0.95 \\
\hline$X_{\mathrm{Ab}}$ & 0.02 & 0.03 & 0.11 & 0.17 & 0.05 & 0.04 & 0.04 & 0.04 \\
\hline
\end{tabular}

*Pre-normalization totals.

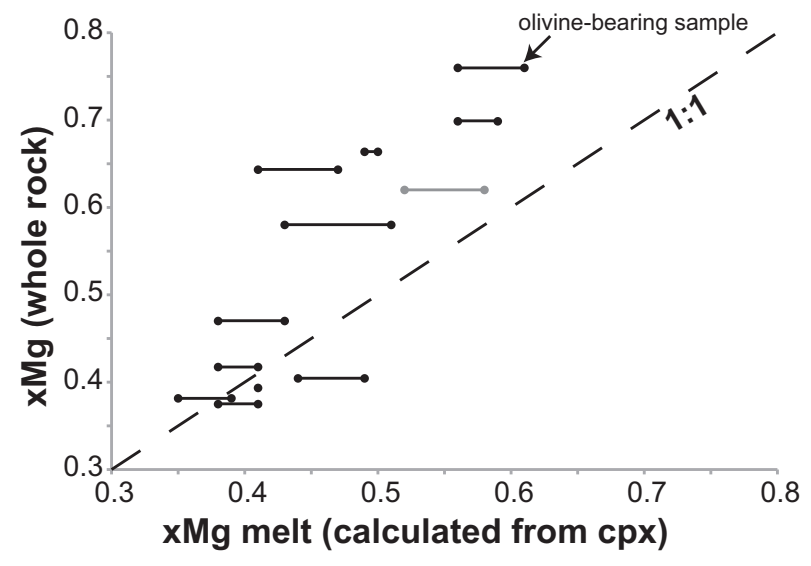

Fig. 11. Whole-rock $X_{\mathrm{Mg}}\left[\mathrm{Mg} /\left(\mathrm{Mg}+\mathrm{Fe}_{\text {tot }}\right)\right]$ vs calculated melt $X_{\mathrm{Mg}}$ for mafic to ultramafic rocks in the Valle Fértil mafic zone. Melt $X_{\mathrm{Mg}}$ is calculated from clinopyroxene assuming a $K_{\mathrm{D}}{ }^{\mathrm{cpx}}(\mathrm{Fe}-\mathrm{Mg})=0.27$ (Roeder \& Emslie, 1970). Samples with whole-rock $X_{\mathrm{Mg}}>0.5$ have corresponding melt $X_{\mathrm{Mg}}$ values that are appreciably lower. This suggests that these rocks have accumulated Mg-bearing phases relative to the crystallizing magma. The range of melt $X_{\mathrm{Mg}}$ for single samples reflects multiple clinopyroxene analyses for each sample. One olivinebearing sample is indicated, and the gray sample is from Otamendi et al. (2009b).

extreme isotopic variation of the SVF rocks (Otamendi et al., 2012).

Despite field and isotopic evidence for crustal contributions within the mafic zone, major and trace element data are consistent with crystal fractionation alone (or rather, do not require the addition of a contaminant).
This presents a modeling dilemma because typical assimilation-fractional crystallization (AFC) models assume and predict coupled variations between elemental and isotopic compositions when mantlederived magmas and crustal material are hybridized. With an increasing crustal isotopic signature, an increase in $\mathrm{K}_{2} \mathrm{O}$ and $\mathrm{SiO}_{2}$ and a decrease in $\mathrm{CaO}$ are expected. Crustal contamination is also expected to exert an influence on trace element concentrations, in particular incompatible elements such as $\mathrm{Rb}, \mathrm{Ba}, \mathrm{Nb}$, and REE. As mentioned above, rocks from the SVF silicic and intermediate zones (above the mafic zone) preserve such elemental and isotopic coupling to some extent, and in particular display a high-K trend that is interpreted to be the product of assimilation and fractional crystallization. There are a few mafic zone tonalite samples that are compositionally suspect with respect to their major and trace elements (elevated $\mathrm{K}_{2} \mathrm{O}$, $\mathrm{Rb}$ and $\mathrm{Ba}$; low $\mathrm{CaO}$; presence of biotite; e.g. FA-09-23, FA-10-10, FA-11-19), but either these samples are near the top of the mafic section or the field relations demonstrate an intimate association with country-rock. Most mafic zone rocks-gabbronorites and tonalites alikelack major and trace element crustal signatures. The mafic zone is largely devoid of elements that are incompatible in the plagioclase- and pyroxene-dominant assemblage.

We propose that elemental and isotopic decoupling can be understood as a product of time-transgressive assimilation, fractional crystallization, and melt loss in the lower crust. In the earliest stages of the SVF 
Table 9: Fractional crystallization model for SVF mafic zone rocks (see text for details)

\begin{tabular}{|c|c|c|c|c|c|c|c|c|c|c|c|}
\hline & & Cumulc & & & & & $\%$ Rem & & & & \\
\hline & Parent & 1 & 2 & 3 & 4 & 5 & 0.2 & 0.15 & 0.1 & 0.3 & 0.3 \\
\hline $\mathrm{SiO}_{2}$ & 50.9 & 44.6 & 48.9 & $45 \cdot 8$ & 44.0 & 49.9 & 52.5 & 53.2 & 54.0 & $58 \cdot 3$ & 61.9 \\
\hline $\mathrm{TiO}_{2}$ & 0.807 & 0.20 & 0.52 & 0.97 & 1.38 & 1.31 & 0.96 & 1.04 & 1.04 & 0.90 & 0.72 \\
\hline $\mathrm{Al}_{2} \mathrm{O}_{3}$ & $17 \cdot 18$ & $17 \cdot 66$ & 18.08 & 17.56 & $17 \cdot 79$ & $18 \cdot 61$ & 17.06 & 16.88 & 16.81 & $16 \cdot 38$ & 15.43 \\
\hline $\mathrm{FeO}_{\mathrm{t}}$ & 10.53 & 9.05 & 9.29 & 13.08 & 15.66 & $12 \cdot 40$ & 10.90 & $11 \cdot 18$ & 10.97 & 8.95 & 7.48 \\
\hline $\mathrm{MnO}$ & 0.242 & 0.14 & 0.19 & 0.24 & 0.25 & 0.24 & 0.27 & 0.28 & 0.28 & 0.30 & 0.33 \\
\hline $\mathrm{MgO}$ & $7 \cdot 48$ & 15.93 & 8.96 & $8 \cdot 18$ & $6 \cdot 80$ & 5.05 & $5 \cdot 37$ & 4.74 & 4.35 & 3.30 & 2.55 \\
\hline $\mathrm{CaO}$ & $10 \cdot 44$ & 11.63 & 12.49 & 12.53 & $12 \cdot 37$ & 9.44 & $10 \cdot 15$ & 9.73 & 9.42 & $8 \cdot 16$ & 7.61 \\
\hline $\mathrm{Na}_{2} \mathrm{O}$ & 1.88 & 0.61 & $1 \cdot 16$ & 1.23 & 1.33 & $2 \cdot 36$ & $2 \cdot 20$ & 2.39 & 2.51 & 3.02 & $3 \cdot 31$ \\
\hline $\mathrm{K}_{2} \mathrm{O}$ & 0.40 & 0.17 & 0.30 & 0.29 & 0.28 & 0.42 & 0.45 & 0.48 & 0.50 & 0.60 & 0.68 \\
\hline $\mathrm{P}_{2} \mathrm{O}_{5}$ & 0.095 & 0.03 & 0.07 & 0.11 & 0.19 & 0.28 & 0.11 & 0.12 & 0.12 & 0.09 & 0.01 \\
\hline$X_{\mathrm{Mg}}$ & 0.56 & 0.76 & 0.63 & 0.53 & 0.44 & 0.42 & 0.51 & 0.49 & 0.48 & 0.50 & 0.54 \\
\hline $\mathrm{Sc}$ & $35(52)$ & 30 & 43 & 51 & 51 & 39 & 58 & 60 & 61 & 66 & 77 \\
\hline V & 267 & 99 & 217 & 370 & 495 & 289 & 310 & 326 & 321 & 246 & 228 \\
\hline $\mathrm{Ba}$ & 90 & 56 & 63 & 71 & 71 & 141 & 99 & 105 & 109 & 125 & 118 \\
\hline $\mathrm{Rb}$ & 4.50 & 4.7 & 6.5 & $4 \cdot 1$ & $4 \cdot 1$ & 7.9 & 4.4 & $4 \cdot 1$ & $4 \cdot 1$ & $4 \cdot 1$ & $2 \cdot 4$ \\
\hline $\mathrm{Sr}$ & 190 (155) & 155 & 167 & 188 & 199 & 231 & 199 & 204 & 206 & 209 & 200 \\
\hline $\mathrm{Zr}$ & 65 & 19 & 27 & 34 & 38 & 171 & 76 & 85 & 91 & 114 & 89 \\
\hline$\vec{Y}$ & $17(31)$ & 5 & 17 & 24 & 21 & 28 & 20 & 21 & 20 & 20 & 17 \\
\hline $\mathrm{Nb}$ & 4.72 & 1.0 & 2.5 & 3.9 & 4.6 & 8.8 & 5.7 & $6 \cdot 2$ & 6.5 & $7 \cdot 3$ & $6 \cdot 7$ \\
\hline $\mathrm{Ta}$ & 0.17 & 0.04 & 0.08 & 0.15 & $0 \cdot 16$ & 0.32 & 0.20 & 0.22 & 0.23 & 0.26 & 0.24 \\
\hline La & $10(13)$ & 1.9 & $7 \cdot 7$ & 8.6 & 8.7 & $16 \cdot 3$ & 12.0 & $12 \cdot 8$ & $13 \cdot 3$ & $15 \cdot 2$ & 14.8 \\
\hline $\mathrm{Ce}$ & $21(30)$ & 4.3 & 18.4 & $22 \cdot 2$ & 21.7 & 38.0 & 25.2 & $26 \cdot 4$ & $26 \cdot 8$ & $29 \cdot 1$ & 25.2 \\
\hline $\mathrm{Pr}$ & 4.13 & 0.6 & 2.6 & 3.3 & 3.2 & 5.2 & 5.0 & 5.4 & 5.7 & $6 \cdot 8$ & $7 \cdot 4$ \\
\hline $\mathrm{Nd}$ & $13(18.5)$ & 2.5 & 11.3 & $14 \cdot 7$ & $14 \cdot 2$ & $22 \cdot 8$ & $15 \cdot 6$ & $16 \cdot 4$ & $16 \cdot 6$ & $17 \cdot 6$ & $15 \cdot 3$ \\
\hline $\mathrm{Sm}$ & $3(5)$ & 0.7 & 2.9 & 3.9 & 3.7 & 5.5 & 3.6 & 3.7 & 3.7 & 3.7 & 2.9 \\
\hline Eu & $1.1(1.3)$ & 0.3 & 0.8 & $1 \cdot 1$ & $1 \cdot 1$ & 1.6 & 1.3 & 1.4 & 1.4 & 1.6 & 1.6 \\
\hline $\mathrm{Gd}$ & $3.4(5.5)$ & 0.8 & 3.0 & $4 \cdot 1$ & $3 \cdot 8$ & 5.5 & $4 \cdot 1$ & 4.2 & 4.3 & 4.4 & 4.0 \\
\hline $\mathrm{Tb}$ & 0.97 & 0.1 & 0.5 & 0.7 & 0.6 & 0.9 & 1.2 & 1.3 & 1.4 & 1.7 & 2.0 \\
\hline Dy & $3.5(6.2)$ & 0.8 & 3.2 & $4 \cdot 3$ & 3.9 & $5 \cdot 4$ & 4.2 & 4.3 & 4.3 & 4.5 & $4 \cdot 2$ \\
\hline Ho & 1.29 & 0.2 & 0.7 & 0.9 & 0.8 & 1.1 & 1.6 & 1.7 & 1.8 & $2 \cdot 2$ & 2.7 \\
\hline $\mathrm{Er}$ & 3.52 & 0.5 & 1.9 & $2 \cdot 4$ & 2.2 & 2.9 & 4.3 & 4.7 & 4.9 & $6 \cdot 1$ & 7.5 \\
\hline $\mathrm{Tm}$ & 0.51 & 0.1 & 0.3 & 0.3 & 0.3 & 0.4 & 0.6 & 0.7 & 0.7 & 0.9 & 1.1 \\
\hline $\mathrm{Yb}$ & $1.6(3.1)$ & 0.4 & 1.8 & $2 \cdot 2$ & 1.9 & 2.5 & 1.9 & 1.9 & 1.9 & 1.9 & 1.6 \\
\hline Lu & $0.25(0.48)$ & 0.1 & 0.3 & 0.3 & 0.3 & 0.4 & 0.6 & 0.6 & 0.7 & 0.8 & 1.0 \\
\hline $\mathrm{U}$ & 0.25 & 0.11 & 0.12 & 0.09 & 0.09 & 0.22 & 0.29 & 0.32 & 0.35 & 0.46 & 0.55 \\
\hline Th & 1.85 & 0.4 & 1.0 & 0.4 & 0.4 & 1.2 & $2 \cdot 2$ & $2 \cdot 4$ & 2.7 & 3.6 & 4.6 \\
\hline $\mathrm{Hf}$ & 1.66 & 0.3 & 0.6 & 1.0 & 1.3 & 4.0 & 2.0 & $2 \cdot 2$ & $2 \cdot 4$ & $2 \cdot 8$ & $2 \cdot 3$ \\
\hline
\end{tabular}

*Numbers in parentheses are actual calculated values. Numbers used in the model appear first.

magmatism when the thermal regime was relatively cool, and in areas where magma flux was low, any assimilation that occurred was incomplete and preserved in the mafic zone rocks (in both gabbronorites and tonalite). With continued mafic input, crustal melting was probably extensive, as expressed by large amounts of diatextite in neighboring septa, but the high temperature and increasingly mafic-dominated compositions would limit the stability of minerals that partition crustally contributed, incompatible major and trace elements (Barnes, 1986; Reiners et al., 1995). Prolonged or punctuated periods of magma injection, crystallization, assimilation, and melt segregation would thus result in crustally contributed major and trace elements being exported from the mafic zone. Throughout this process, however, mineral accumulation would preserve crustally derived isotopic signatures-the only detectable evidence of the assimilant. Elemental and isotopic decoupling, then, is conceivably an expected byproduct of prolonged mafic magmatism involving assimilation accompanied by extremely efficient crystal-melt segregation.

\section{The anatomy of a MASH zone revealed}

We interpret the mafic zone as the site at which mafic magmas stalled, assimilated crust, partially crystallized and remobilized previously stalled magmas. Such a scenario is consistent with the MASH process (melting, assimilation, storage, homogenization) proposed by Hildreth \& Moorbath (1988). We document such a process from a field-based geological perspective. Although assimilation and crystallization probably occurred at multiple levels within the SVF system, the mafic zone probably represents the first and perhaps principal stage of differentiation of mantle-derived melts prior to subsequent reintrusion or eruption. The most primitive components are preserved as ultramafic cumulates that occur sporadically throughout the mafic section, forming discrete bodies that range in size from tens of meters to $\sim 400 \mathrm{~m}$ thick (by $<1 \mathrm{~km}$ wide). We interpret the dimensions of these ultramafic bodies as indicative of the size of possible parental magma batches at this level of the system (actual batch sizes must have been much larger as these ultramafic cumulates are interpreted to have lost $\sim 80 \%$ melt). Although not 


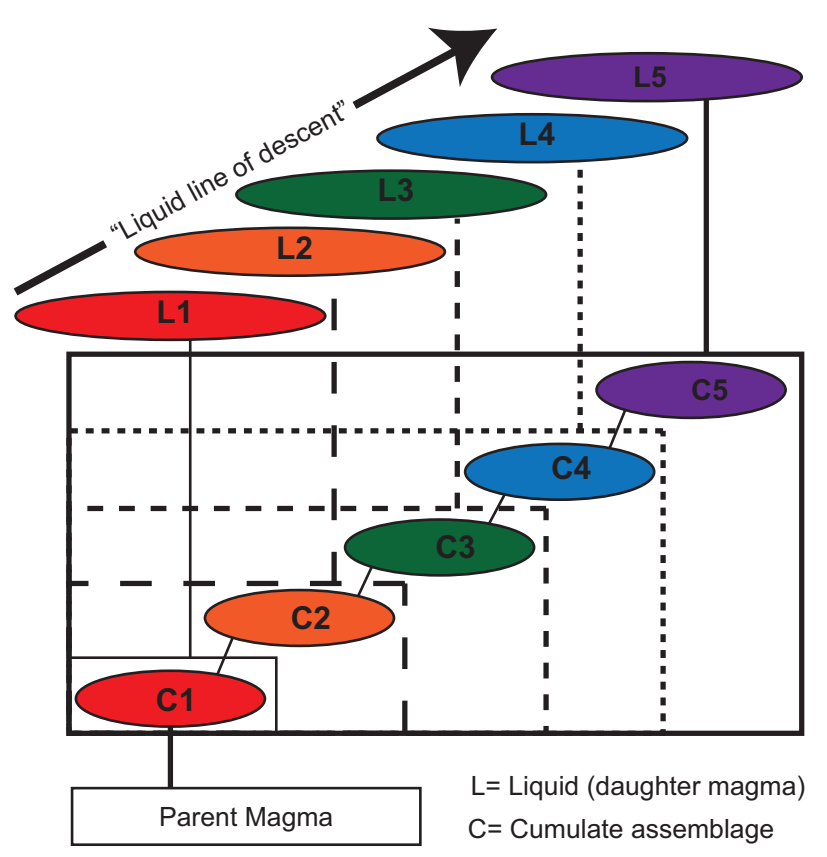

Fig. 12. Schematic diagram of the fractionation model. From a parent magma, a cumulate assemblage $(\mathrm{C} 1)$ is subtracted and a daughter liquid (L1) is produced. From the L1 daughter liquid, a second cumulate (C2) is subtracted and a second daughter liquid (L2) is produced. From that L2 liquid, a third cumulate (C3) is subtracted, yielding a third daughter liquid (L3), and so on. In the diagram, daughter liquids are connected by patterned lines with their total crystal residues, which are enclosed by a box with the same pattern. For example, the daughter magma L1 has the crystal residue of $\mathrm{C} 1$. So, $\mathrm{L} 1+\mathrm{C} 1=$ parent magma. However, the crystal residue of the daughter magma L4 includes $\mathrm{C} 1, \mathrm{C} 2, \mathrm{C} 3$, and C4. Therefore, $\mathrm{L} 4+\mathrm{C} 1+\mathrm{C} 2+\mathrm{C} 3+$ $\mathrm{C} 4=$ parent magma.

representing the amount of molten material at any given moment, the ultramafic cumulates, nonetheless, might indicate the relative volumes of magmatic pulses in pluton-building episodes. The gabbronorites, then, must represent residual compositions after repeated magma injection, back-mixing, fractionation and melt expulsion. Tonalites represent melts that were separated from these gabbronorite cumulate assemblages.

This simplified framework of cumulates and segregated melt produces very complex field relationships. In the field, samples were selected from relatively homogeneous domains (or at least portions of outcrop). However, many of the outcrops we observe in the SVF mafic zone are extremely heterogeneous (including multiple generations of enclaves, some with cumulate compositions) and lack visible contacts between modally distinct domains. The internal organization of the mafic zone seems to reflect a prolonged, chaotic assembly process. Mafic magmas intruded variably cumulate tonalites, tonalite magmas intruded variably cumulate gabbronorites, and these processes occurred both high and low within the mafic zone. Gradational contacts between different gabbroic and tonalitic rocks suggest that re-intrusion blended existing cumulates with new intrusions and involved near-simultaneous melt extraction.
In general, field relations in the SVF mafic zone share some features with those described at the Onion Valley sill complex in the Sierra Nevada (Sisson et al., 1996). The Onion Valley complex is composed of basaltic to basaltic andesite sills that intruded an existing granodiorite matrix. The sills are of variable thickness and include multiply injected swarm complexes, some of which contain olivine, are modally layered, and are interpreted as cumulates. However, owing in part to their shallow emplacement depth ( $\sim 2$ kbar pressure), the Onion Valley sills have several characteristics that the SVF mafic magmas lack. In particular, the Onion Valley sills have chilled margins and miarolitic cavities; many are interpreted to preserve melt compositions (Sisson et al., 1996). However, the general interpretation that the mafic sills invaded a solid to partially molten silicic crystal mush is consistent with our observations for the SVF. In the SVF the invading mafic sills were emplaced into, and fractionated within, tonalites that are themselves products of the same process (Fig. 15). The SVF mafic complex also shares features with the Woolley Creek batholith of the Klamath Mountains, California, with the important exception that at Woolley Creek there was not as much homogenization between new magma batches as observed at SVF (Coint et al., 2013).

Discrete compositional domains persisted in the SVF mafic complex, despite prolonged, open-system mushblending; however, compositional variation is present within single domains. For example, any given 'gabbronorite' domain on the map (Fig. 16) contains a spectrum of compositions. Additionally, specific compositional varieties (of gabbronorite or tonalite) are not spatially confined, but are found seemingly randomly distributed within the mafic zone. Although our petrogenetic model is consistent with a residue-segregated melt relationship for the gabbronorites and tonalites the actual process is almost certainly much more complex. Tonalites and gabbronorites are probably composed of meltmineral combinations that, although roughly appearing to be an equilibrium assemblage, may in fact be hybrids. Fractional crystallization is plausibly the dominant mechanism responsible for compositional variation, but compositional trends are also probably in part due to back-mixing, incomplete melt extraction and melt reinfiltration.

\section{CONCLUSIONS}

The SVF mafic zone represents the upper part of a MASH zone in which mafic, mantle-derived magmas penetrated the crust. The compositional spectrum of SVF mafic zone rocks is consistent with a crystal fractionation process in which the mafic to ultramafic rocks-mostly various types of gabbronorite-are cumulates, and the tonalites are the corresponding silicic segregates. It is clear from the $\mathrm{Sr}$ and $\mathrm{Nd}$ isotope data that assimilation of crustal rocks occurred at all levels within the SVF system, and possibly also within the mantle wedge. Major and trace element evidence for 

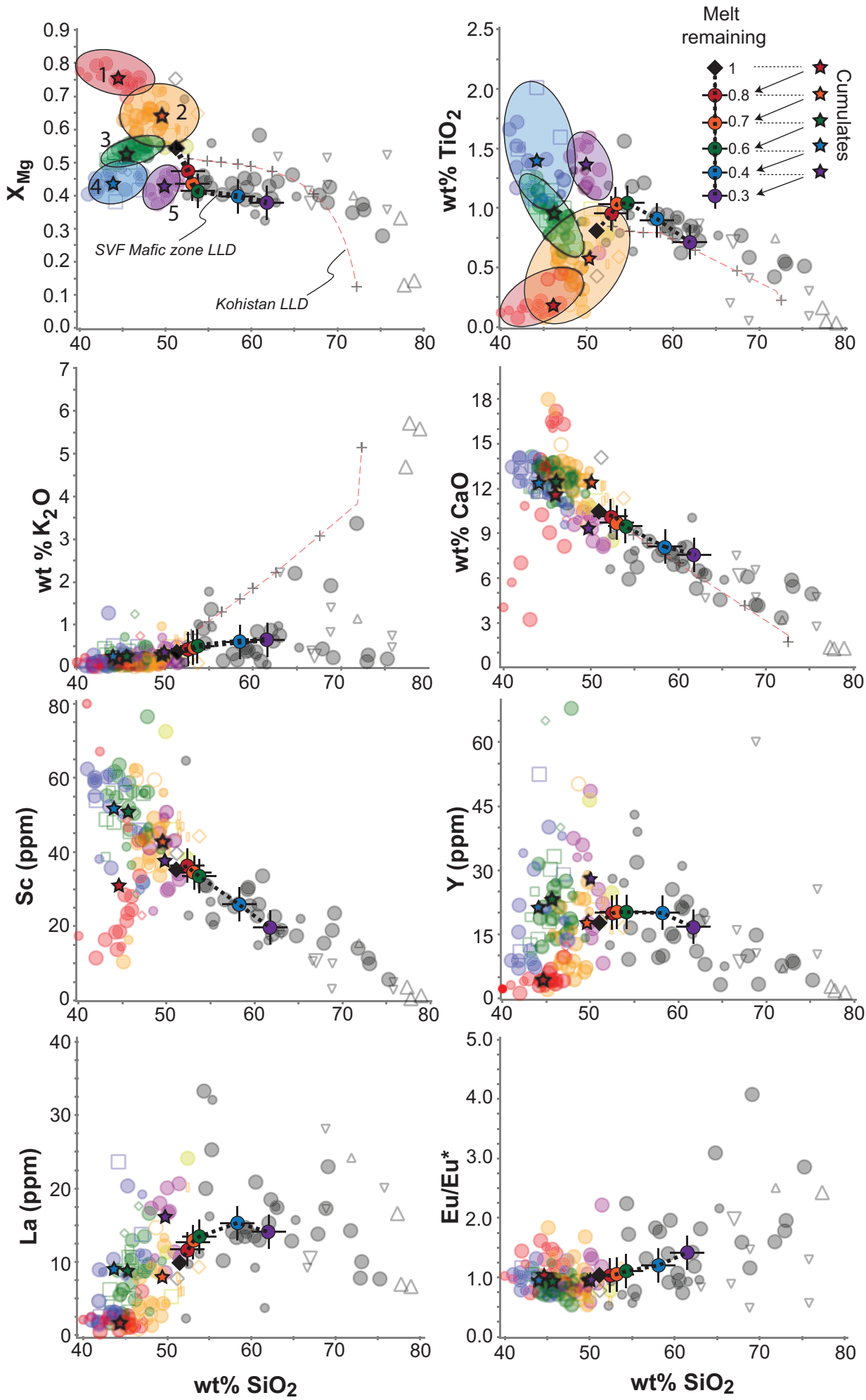

Fig. 13. Variation of selected elements vs $\mathrm{SiO}_{2}$ and the fractional crystallization model for the SVF mafic zone rocks. The starting composition is indicated by a black diamond. Model cumulate assemblages are indicated by stars and are the calculated averages of the translucent data points of the same color (cumulate groups used for averaging are circled in plots of $X_{\mathrm{Mg}}$ and TiO ${ }_{2}$ ). From the starting composition, the cumulates are progressively subtracted in the order 1-5 (labeled in the $X_{\mathrm{Mg}}$ and $\mathrm{TiO}_{2}$ vs $\mathrm{SiO}_{2}$ plots). Bold colored circles with crosses are the modeled daughter magma compositions, the color of which corresponds to the cumulate assemblage subtracted during its fractionation step. The melt remaining $(F)$ at each interval of fractionation is indicated. Modeled liquid lines of descent for selected elements from the Kohistan arc system are also shown (Jagoutz, 2010). In general, there is good agreement between our model liquid lines of descent and those of Jagoutz (2010), with the notable exception of $\mathrm{K}_{2} \mathrm{O}$. (See text for additional details of our model.) $X_{\mathrm{Mg}}=\mathrm{Mg} /\left(\mathrm{Mg}+\mathrm{Fe}_{\mathrm{tot}}\right)$. LLD, liquid line of descent. 

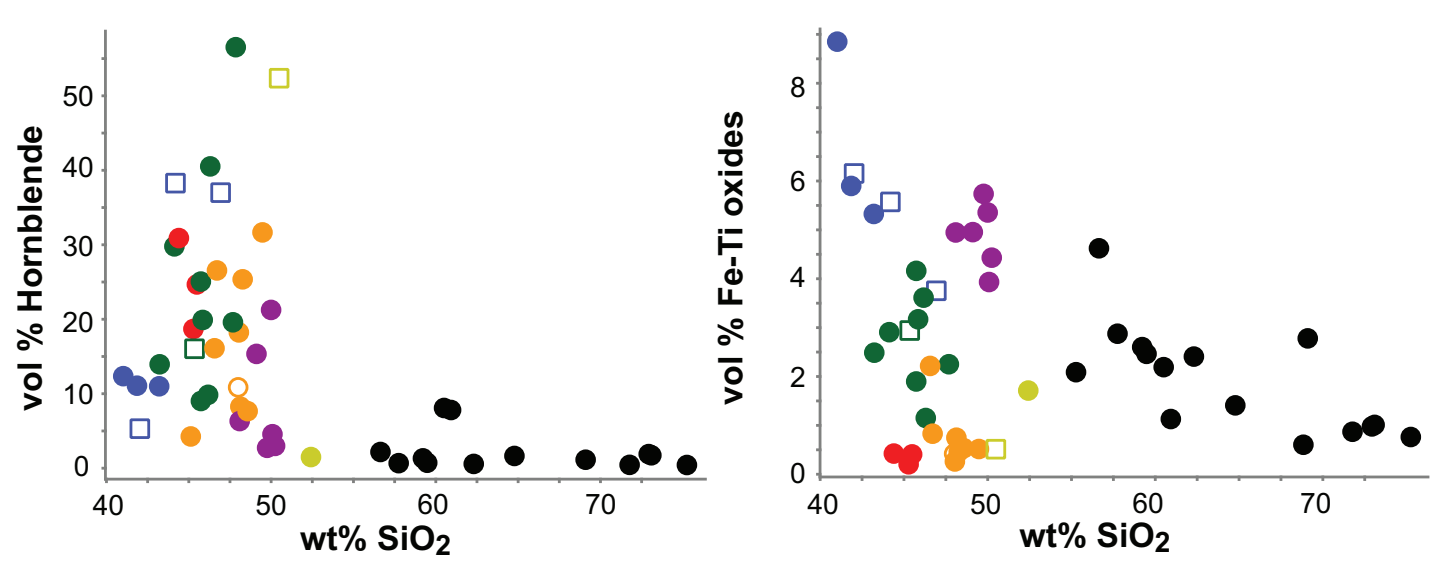

Fig. 14. Amphibole (hornblende) and Fe-Ti oxides (magnetite and ilmenite) modes vs weight per cent $\mathrm{SiO}_{2}$ for the $\mathrm{SVF}$ mafic zone rocks. It should be noted that the rocks with lowest $\mathrm{SiO}_{2}$ are those with the highest volume per cent Fe-Ti oxides. Symbols are as in Fig. 6. Open squares, dikes; open circles, blob-like inclusions.

Time 1
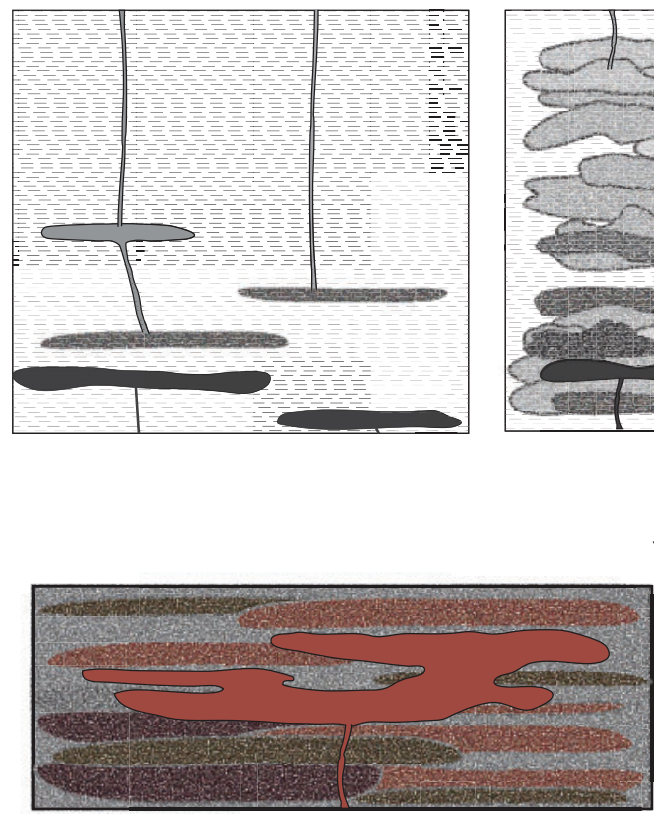

Injection, limited mixing
Time 2

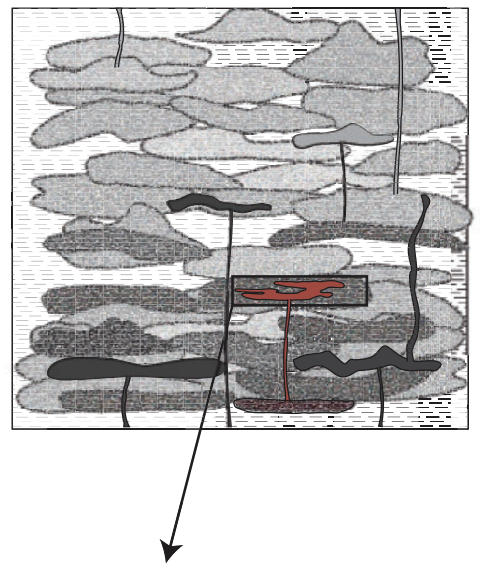

Time 3

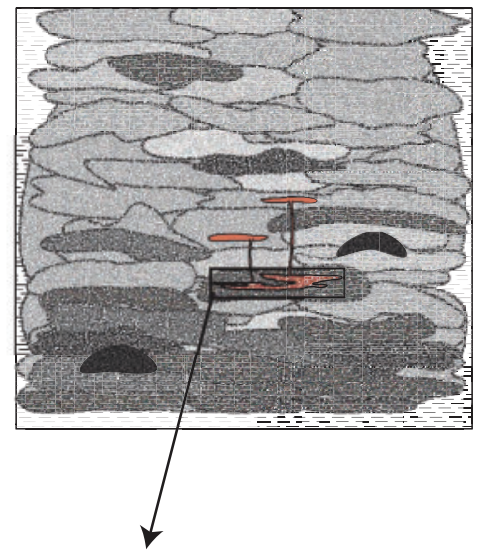

Fig. 15. Schematic illustration of generalized assembly of the Sierra Valle Fértil mafic zone. A prolonged, chaotic scenario involving MASH-like processes is envisioned. Efficient melt extraction leaves behind distinct cumulate packages, but thermal annealing probably obscures initial intrusion and extraction contacts. Discrete compositional domains persist, although these domains themselves appear to comprise multiple sheets of distinct composition.

assimilation is sparse within the mafic zone rocks, suggesting that the incompatible components of the assimilated crust were flushed out with extraordinary efficiency during prolonged magmatism. The silicic liquid extracted from the MASH zone was expelled upward in the system, giving rise to compositionally stratified arc crust almost completely reconstituted by magmatism. Despite a large-scale stratification consisting of a mafic deep crust, intermediate mid-crust and silicic upper crust (Otamendi et al., 2012), the SVF mafic complex is characterized by considerable heterogeneity at smaller scales. This is indicative of very complex temporal and spatial assembly processes, all sharing a common thermal environment at the prograde peak. Models for arc processes that invoke a 'ball and stick' morphology-where the mid-crust is transparent to the petrogenetic processes, and evolved magmas leave the deep crust and rise superheated to the upper crust-are not supported by this field example. It is also worth restating that there is no field evidence for the production of voluminous silicic magmas by simple melting of mafic precursors; the mid-crustal intermediate magmas 


\section{Composition maps of the SVF Mafic Zone}

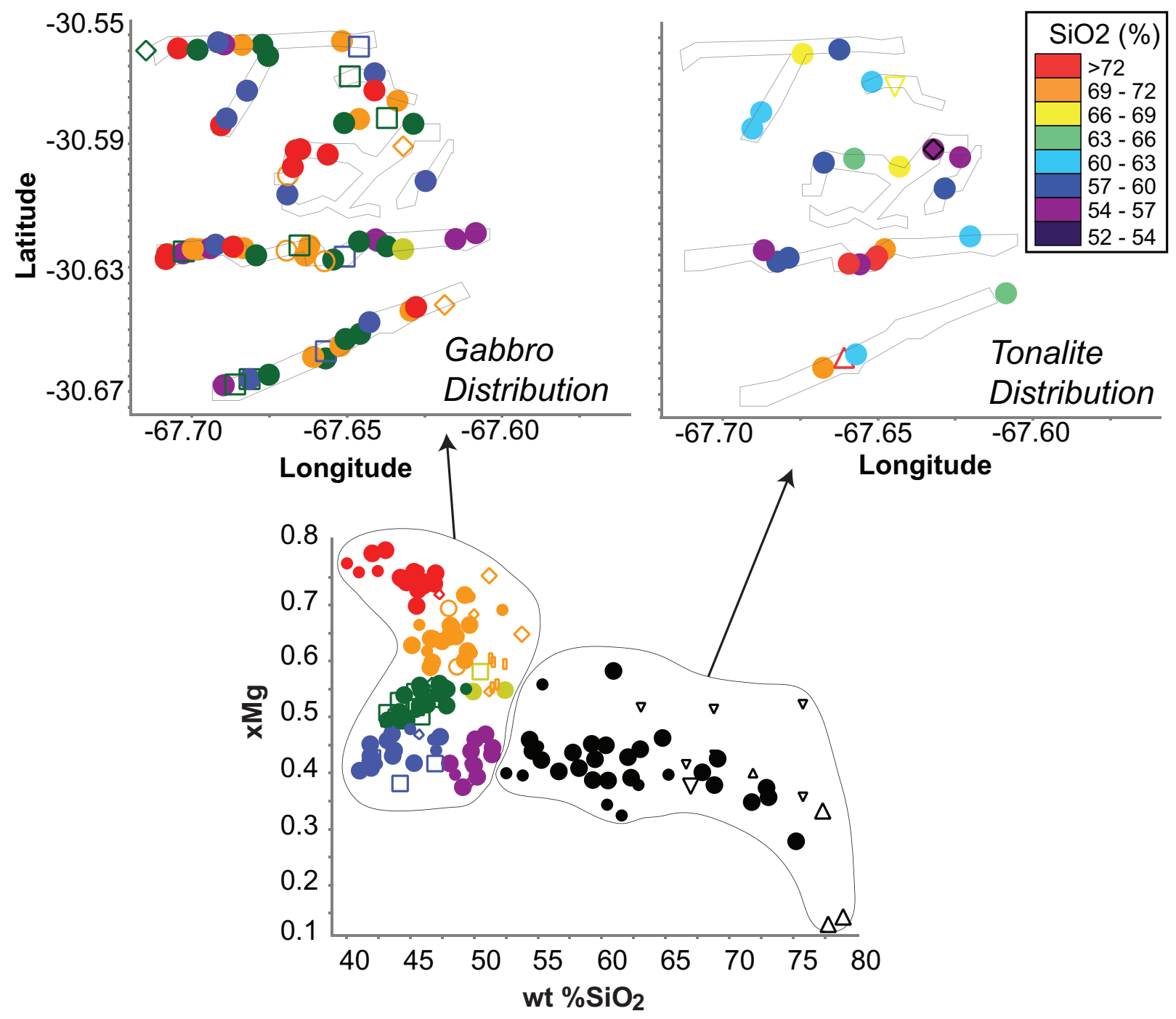

Fig. 16. Spatial distributions (latitude-longitude) of compositional variants of gabbro and tonalite in the SVF mafic zone. Gabbroic rocks are plotted with respect to their latitude-longitude, color-coded according to their position in the $X_{\mathrm{Mg}}$ vs $\mathrm{SiO}_{2}$ plot. Tonalites, shown in black on the $X_{\mathrm{Mg}}$ vs $\mathrm{SiO}_{2}$ plot, are color coded based on their $\mathrm{SiO}_{2}$ content in the latitude-longitude plot. Outlines of canyons are from Fig. 2.

at the SVF appear to be the product of crystal fractionation and some assimilation.

\section{ACKNOWLEDGEMENTS}

We express our appreciation to Tenley Banik, Nacho Baliani, Alina Tibaldi, Chad Deering, Olivier Bachmann, Dennis Geist, and Karen Harpp for their help and companionship in the field and for fruitful petrological discussion. Andrew Hill, Jerome Garcia, and Sonny Blount also provided critical stimuli for discussions in the field and in the laboratory. Keith Putirka, Oliver Jagoutz, and an anonymous reviewer provided extremely helpful comments on an earlier version of this paper. We thank Jim Beard for editorial handling.

\section{FUNDING}

This work was supported by a grant from the National Science Foundation, EAR-1049884 to G.W.B. M.N.D. was supported by COSA, an ExxonMobil-University of Arizona initiative. J.E.O. was funded by ANPCyT Argentina through grants PICT 01904/07 and 00453/10, and SeCyT-UNRC.

\section{SUPPLEMENTARY DATA}

Supplementary data for this paper are available at Journal of Petrology online.

\section{REFERENCES}

Acenolaza, F. G., Miller, H. \& Toselli, A. J. (2002). ProterozoicEarly Paleozoic evolution in western South America-a discussion. Tectonophysics 354, 121-137. 
Andrew, A., Armstrong, R. L. \& Runkle, D. (1991). Neodymiumstrontium-lead isotopic study of Vancouver Island igneous rocks. Canadian Journal of Earth Sciences 28, 1744-1752.

Annen, C., Blundy, J. D. \& Sparks, R. S. J. (2006). The genesis of intermediate and silicic magmas in deep crustal hot zones. Journal of Petrology 47, 505-539.

Bachl, C. A., Miller, C. F., Miller, J. S. \& Faulds, J. E. (2001). Construction of a pluton: Evidence from an exposed cross section of the Searchlight pluton, Eldorado Mountains, Nevada. Geological Society of America Bulletin 113, 1213-1228.

Barboza, S. A. \& Bergantz, G. W. (1996). Dynamic model of dehydration melting motivated by a natural analogue: applications to the Ivrea-Verbano zone, northern Italy. Transactions of the Royal Society of Edinburgh 87, 23-31.

Barboza, S. A. \& Bergantz, G. W. (2000). Metamorphism and anatexis in the mafic complex contact aureole, Ivrea Zone, Northern Italy. Journal of Petrology 41, 1307-1327.

Barnes, S. (1986). The effect of trapped liquid crystallization on cumulus mineral compositions in layered intrusions. Contributions to Mineralogy and Petrology 93, 524-531.

Beard, J. S. (1995). Experimental, geological, and geochemical constraints on the origins of low-K silicic magmas in oceanic arcs. Journal of Geophysical Research: Solid Earth 100, 15593-15600.

Beard, J. S. (1997). Geochemistry and petrogenesis of tonalite dikes in the Smith River allochthon, south-central Virginia. In: Sinha, A.K, Whalen, J.B., Hogan, J.P., (eds) The Nature of Magmatism in the Appalachian Orogen. Geological Society of America, Memoirs 191, 75-86.

Beard, J. S. \& Lofgren, G. E. (1989). Effects of water on the composition of partial melts of greenstone and amphibolite. Science 244, 195-197.

Bergantz, G. W. (1991). Physical and chemical characterization of plutons. In: Kerrick, D. M. (ed.) Contact Metamorphism. Mineralogical Society of America, Reviews in Mineralogy 26, 13-42.

Bergantz, G. W. (1992). Conjugate solidification and melting in multicomponent open and closed systems. International Journal of Heat and Mass Transfer 35, 533-543.

Bucholz, C., Jagoutz, O., Schmidt, M. \& Sambuu, O. (2014). Fractional crystallization of high-K arc magmas: biotite- versus amphibole-dominated fractionation series in the Dariv Igneous Complex, Western Mongolia. Contributions to Mineralogy and Petrology 168, 1-28.

Burg, J. P., Bodinier, J. L., Chaudhry, S., Hussain, S. \& Dawood, H. (1998). Infra-arc mantle-crust transition and intra-arc mantle diapirs in the Kohistan Complex (Pakistani Himalaya): petro-structural evidence. Terra Nova 10, 74-80.

Calvert, A. J., Klemperer, S. L., Takahashi, N. \& Kerr, B. C. (2008). Three-dimensional crustal structure of the Mariana island arc from seismic tomography. Journal of Geophysical Research: Solid Earth 113 doi: 10.1029/ 2007JB004939.

Casquet, C., Rapela, C. W., Pankhurst, R. J., Baldo, E., Galindo, C., Fanning, C. M. \& Dahlquist, J. (2012). Fast sediment underplating and essentially coeval juvenile magmatism in the Ordovician margin of Gondwana, Western Sierras Pampeanas, Argentina. Gondwana Research 22, 664-673.

Claeson, D. \& Meurer, W. (2004). Fractional crystallization of hydrous basaltic 'arc-type' magmas and the formation of amphibole-bearing gabbroic cumulates. Contributions to Mineralogy and Petrology 147, 288-304.

Claiborne, L. L., Miller, C. F., Walker, B. A., Wooden, J. L., Mazdab, F. K. \& Bea, F. (2006). Tracking magmatic processes through $\mathrm{Zr} / \mathrm{Hf}$ ratios in rocks and $\mathrm{Hf}$ and $\mathrm{Ti}$ zoning in zircons: An example from the Spirit Mountain batholith, Nevada. Mineralogical Magazine 70, 517-543.
Clemens, J. D., Stevens, G. \& Farina, F. (2011). The enigmatic sources of I-type granites: The peritectic connexion. Lithos 126, 174-181.

Coint, N., Barnes, C. G., Yoshinobu, A. S., Chamberlain, K. R. \& Barnes, M. A. (2013). Batch-wise assembly and zoning of a tilted calc-alkaline batholith: Field relations, timing, and compositional variation. Geosphere 9, 1729-1746.

Coleman, D. S., Bartley, J. M., Glazner, A. F. \& Pardue, M. J. (2012). Is chemical zonation in plutonic rocks driven by changes in source magma composition or shallow-crustal differentiation? Geosphere 8, 1568-1587.

Collo, G., Astini, R. A., Cawood, P. A., Buchan, C. \& Pimentel, M. (2009). U-Pb detrital zircon ages and $\mathrm{Sm}-\mathrm{Nd}$ isotopic features in low-grade metasedimentary rocks of the Famatina belt: implications for late Neoproterozoic-early Palaeozoic evolution of the proto-Andean margin of Gondwana. Journal of the Geological Society, London 166, 303-319.

Conrad, W. K. \& Kay, R. W. (1984). Ultramafic and mafic inclusions from Adak Island-crystallization history, and implications for the nature of primary magmas and crustal evolution in the Aleutian Arc. Journal of Petrology 25, 88-125.

Cristofolini, E. A., Otamendi, J. E., Ducea, M. N., Pearson, D. M., Tibaldi, A. M. \& Baliani, I. (2012). Detrital zircon U-Pb ages of metasedimentary rocks from Sierra de Valle Fértil: Entrapment of Middle and Late Cambrian marine successions in the deep roots of the Early Ordovician Famatinian arc. Journal of South American Earth Sciences 37, 77-94.

Cristofolini, E. A., Otamendi, J. E., Walker, B. A., Jr, Tibaldi, A. M., Armas, P., Bergantz, G. W. \& Martino, R. D. (2014). A Middle Paleozoic shear zone in the Sierra de Valle Fértil, Argentina: Records of a continent-arc collision in the Famatinian margin of Gondwana. Journal of South American Earth Sciences 56, 170-185.

Cui, Y. \& Russell, J. K. (1995). Nd-Sr-Pb isotopic studies of the southern Coast Plutonic Complex, southwestern British Columbia. Geological Society of America Bulletin 107, 127-138.

Davidson, J., Turner, S., Handley, H., Macpherson, C. \& Dosseto, A. (2007). Amphibole 'sponge' in arc crust? Geology 35, 787-790.

DeBari, S. M. (1994). Petrogenesis of the Fimbala gabbroic intrusion, northwestern Argentina, a deep crustal syntectonic pluton in a continental magmatic arc. Journal of Petrology 35, 679-713.

DeBari, S. M. \& Coleman, R. G. (1989). Examination of the deep levels of an island arc: Evidence from the Tonsina Ultramafic-Mafic Assemblage, Tonsina, Alaska. Journal of Geophysical Research: Solid Earth 94, 4373-4391.

DeBari, S. M. \& Mortensen, J. (1994). The Jurassic Bonanza arc, Vancouver Island, Canada: A window into the deep crust of an island arc. Geological Society of America, Abstracts with Programs, Annual Meeting A-332.

DeBari, S. M., Anderson, R. G. \& Mortensen, J. K. (1999). Correlation among lower to upper crustal components in an island arc: the Jurassic Bonanza arc, Vancouver Island, Canada. Canadian Journal of Earth Sciences 36, 1371-1413.

Dessimoz, M., Muntener, O. \& Ulmer, P. (2012). A case for hornblende dominated fractionation of arc magmas: the Chelan Complex (Washington Cascades). Contributions to Mineralogy and Petrology 163, 567-589.

Dhuime, B., Bosch, D., Garrido, C. J., Bodinier, J.-L., Bruguier, O., Hussain, S. S. \& Dawood, H. (2009). Geochemical architecture of the lower- to middle-crustal section of a paleoisland arc (Kohistan complex, Jijal-Kamila area, northern Pakistan): implications for the evolution of an oceanic subduction zone. Journal of Petrology 50, 531-569. 
Dilles, J. H. (1987). Petrology of the Yerington Batholith, Nevada-evidence for evolution of porphyry copper ore fluids. Economic Geology 82, 1750-1789.

Ducea, M. N. \& Saleeby, J. (1998). The age and origin of a thick mafic-ultramafic root from beneath the Sierra Nevada batholith. Contributions to Mineralogy and Petrology 133, 169-185.

Ducea, M. N., Kidder, S. \& Zandt, G. (2003). Arc composition at mid-crustal depths: insights from the Coast Ridge Belt, Santa Lucia Mountains, California. Geophysical Research Letters 30, 1703-1706.

Ducea, M. N., Otamendi, J. E., Bergantz, G., Stair, K. M., Valencia, V. A. \& Gehrels, G. E. (2010). Timing constraints on building an intermediate plutonic arc crustal section: $\mathrm{U}-\mathrm{Pb}$ zircon geochronology of the Sierra Valle Fertil-La Huerta, Famatinian arc, Argentina. Tectonics 29 doi: 10.1029/ 2009 TC002615.

Ducea, M. N., Otamendi, J. E., Bergantz, G. W., Jianu, D. \& Petrescu, L. (2014). The origin and petrologic evolution of the Ordovician Famatinian-Puna arc. In: DeCelles, P.G. et al (eds) Geodynamics of a Cordilleran Orogenic System: The Central Andes of Argentina and Northern Chile. Geological Society of America, Memoirs 212, 125-138.

Ducea, M. N., Saleeby, J. B. \& Bergantz, G. (2015). The architecture, chemistry, and evolution of continental magmatic arcs. Annual Review of Earth and Planetary Sciences 43, 299-331.

Dufek, J. \& Bergantz, G. W. (2005). Lower crustal magma genesis and preservation: a stochastic framework for the evaluation of basalt-crust interaction. Journal of Petrology 46, 2167-2196.

Economos, R. C., Hanzl, P., Hrdlickova, K., Burianek, D., Said, L. O., Gerdes, A. \& Paterson, S. R. (2008). Geochemical and structural constraints on the magmatic history of the Chandman Massif of the eastern Mongolian Altay Range, SW Mongolia. Journal of Geosciences 53, 335-352.

Gallien, F., Mogessie, A., Bjerg, E., Delpino, S., de Machuca, B. C., Thöni, M. \& Klötzli, U. (2010). Timing and rate of granulite facies metamorphism and cooling from multi-mineral chronology on migmatitic gneisses, Sierras de La Huerta and Valle Fértil, NW Argentina. Lithos 114, 229-252.

Greene, A. R., DeBari, S. M., Kelemen, P. K., Blustajn, J. \& Clift, P. D. (2006). A detailed geochemical study of island arc crust: the Talkeetna arc section, south-central Alaska. Journal of Petrology 47, 1051-1093.

Hacker, B. R., Mehl, L., Kelemen, P. B., Rioux, M., Behn, M. D. \& Luffi, P. (2008). Reconstruction of the Talkeetna intraoceanic arc of Alaska through thermobarometry. Journal of Geophysical Research: Solid Earth 11322 doi:10.1029/ 2007JB005208.

Haraguchi, S., Ishii, T., Kimura, J.-I. \& Ohara, Y. (2003). Formation of tonalite from basaltic magma at the Komahashi-Daini Seamount, northern Kyushu-Palau Ridge in the Philippine Sea, and growth of Izu-Ogasawara (Bonin)-Mariana arc crust. Contributions to Mineralogy and Petrology 145, 151-168.

Hildreth, W. (1981). Gradients in silicic magma chambers: implications for lithospheric magmatism. Journal of Geophysical Reserach 86, 10153-110192.

Hildreth, W. \& Moorbath, S. (1988). Crustal contributions to arc magmatism in the Andes of Central Chile. Contributions to Mineralogy and Petrology 98, 455-489.

Hoal, K. O., Stammer, J. G., Appleby, S. K., Botha, J., Ross, J. K. \& Botha, P. W. (2009). Research in quantitative mineralogy: Examples from diverse applications. Minerals Engineering 22, 402-408.

Holbrook, W. S., Lizarralde, D., McGeary, S., Bangs, N. \& Diebold, J. (1999). Structure and composition of the
Aleutian island arc and implications for continental crustal growth. Geology 27, 31-34.

Hoskin, P. W. O. \& Schaltegger, U. (2003). The composition of zircon and igneous and metamorphic petrogenesis. In: Hanchar, J. M. \& Hoskin, P. W. O. (eds) Zircon. Mineralogical Society of America and Geochemical Society, Reviews in Mineralogy and Geochemistry 53, 27-62.

Jagoutz, O. (2010). Construction of the granitoid crust of an island arc. Part II: a quantitative petrogenetic model. Contributions to Mineralogy and Petrology 160, 359-381.

Jagoutz, O., Müntener, O., Burg, J. P., Ulmer, P. \& Jagoutz, E. (2006). Lower continental crust formation through focused flow in $\mathrm{km}$-scale melt conduits: The zoned ultramafic bodies of the Chilas Complex in the Kohistan island arc (NW Pakistan). Earth and Planetary Science Letters 242, 320-342.

Jagoutz, O., Muntener, O., Ulmer, P., Pettke, T., Burg, J.-P., Dawood, H. \& Hussain, S. (2007). Petrology and mineral chemistry of lower crustal intrusions: the Chilas Complex, Kohistan (NW Pakistan). Journal of Petrology 48, 1895-1953.

Jagoutz, O., Müntener, O., Schmidt, M. W. \& Burg, J.-P. (2011). The roles of flux- and decompression melting and their respective fractionation lines for continental crust formation: Evidence from the Kohistan arc. Earth and Planetary Science Letters 303, 25-36.

Johnson, D. M., Hooper, P. R. \& Conrey, R. M. (1999). XRF analysis of rocks and minerals for major and trace elements on a single low dilution Li-tetraborate fused bead. Advances in $X$-ray Analysis 41, 843-867.

Kamiyama, H., Nakajima, T. \& Kamioka, H. (2007). Magmatic stratigraphy of the tilted Tottabetsu plutonic complex, Hokkaido, North Japan: Magma chamber dynamics and pluton construction. Journal of Geology 115, 295-314.

Kawate, S. \& Arima, M. (1998). Petrogenesis of the Tanzawa plutonic complex, central Japan: exposed felsic mid-crust of the Izu-Bonin-Mariana arc. Island Arc 7, 342-358.

Kay, R. W. \& Mahlburg Kay, S. (1993). Delamination and delamination magmatism. Tectonophysics 219, 177-189.

Kidder, S., Ducea, M., Gehrels, G., Patchett, P. J. \& Vervoot, J. (2003). Tectonic and magmatic development of the Salinian Coast Ridge belt. Tectonics 22 doi:10.1029/ 2002TC001409.

Kitamura, K., Ishikawa, M. \& Arima, M. (2003). Petrological model of the northern Izu-Bonin-Mariana arc crust: constraints from high-pressure measurements of elastic wave velocities of the Tanzawa plutonic rocks, central Japan. Tectonophysics 371, 213-221.

Lackey, J. S., Valley, J. W. \& Saleeby, J. B. (2005). Supracrustal input to magmas in the deep crust of Sierra Nevada batholith: Evidence from high- $\delta^{18} \mathrm{O}$ zircon. Earth and Planetary Science Letters 235, 315-330.

Larocque, J. \& Canil, D. (2010). The role of amphibole in the evolution of arc magmas and crust: the case from the Jurassic Bonanza arc section, Vancouver Island, Canada. Contributions to Mineralogy and Petrology 159, 475-492.

Leake, B. E., Woolley, A. R., Arps, C. E. S., Birch, W. D., Gilbert, M. C., Grice, J. D., Hawthorne, F. C., Kato, A., Kisch, H. J., Krivovichev, V. G., Linthout, K., Laird, J., Mandarino, J. A., Maresch, W. V., Nickel, E. H., Rock, N. M. S., Schumacher, J. C., Smith, D. C., Stephenson, N. C. N., Ungaretti, L., Whittaker, E. J. W. \& Guo, Y. (1997). Nomenclature of amphiboles; Report of the Subcommittee on Amphiboles of the International Mineralogical Association, Commission on New Minerals and Mineral Names. American Mineralogist 82, 1019-1037.

Lee, C. T., Rudnick, R. L. \& Brimhall, G. H. (2001). Deep lithospheric dynamics beneath the Sierra Nevada during the Mesozoic and Cenozoic as inferred from xenolith petrology. 
Geochemistry, Geophysics, Geosystems 2 doi:10.1029/ 2001GC000152.

Longo, A. A., Dilles, J. H., Grunder, A. L. \& Duncan, R. (2010). Evolution of calc-alkaline volcanism and associated hydrothermal gold deposits at Yanacocha, Peru. Economic Geology 105, 1191-1240.

Meurer, W. P. \& Claeson, D. T. (2002). Evolution of crystallizing interstitial liquid in an arc-related cumulate determined by LA ICP-MS mapping of a large amphibole oikocryst. Journal of Petrology 43, 607-629.

Miller, C. F. \& Miller, J. S. (2002). Contrasting stratified plutons exposed in tilt blocks, Eldorado Mountains, Colorado River Rift, NV, USA. Lithos 61, 209-224.

Miller, R. B. \& Paterson, S. R. (2001). Construction of mid-crustal sheeted plutons: Examples from the north Cascades, Washington. Geological Society of America Bulletin 113, 1423-1442.

Miller, R. B., Paterson, S. R. \& Matzel, J. P. (2009). Plutonism at different crustal levels: Insights from the $\sim 5-40 \mathrm{~km}$ (paleodepth) North Cascades crustal section, Washington. In: Miller, R. B. \& Snoke, A.W. (eds) Crustal Cross Sections from the Western North American Cordillera and Elsewhere: Implications for Tectonic and Petrologic Processes. Geological Society of America, Special Papers 456, 125-149.

Mirré, J. C. (1976). Descripción Geológica de la Hoja 19e, Valle Fértil, Provincias de San Juan y La Rioja. Servicio Geológico Nacional, pp. 1-70.

Mulcahy, S. R., Roeske, S. M., McClelland, W. C., Nomade, S. \& Renne, P. R. (2007). Cambrian initiation of the Las Piquitas thrust of the western Sierras Pampeanas, Argentina: Implications for the tectonic evolution of the proto-Andean margin of South America. Geology 35, 443-446.

Mulcahy, S. R., Roeske, S. M., McClelland, W. C., Ellis, J. R., Jourdan, F., Renne, P. R., Vervoort, J. D. \& Vujovich, G. I. (2014). Multiple migmatite events and cooling from granulite facies metamorphism within the Famatina arc margin of northwest Argentina. Tectonics 33, 1-25.

Needy, S. K., Anderson, J. L., Wooden, J. L., Fleck, R. J., Barth, A. P., Paterson, S. R., Memeti, V. \& Pignotta, G. S. (2009). Mesozoic magmatism in an upper- to middle-crustal section through the Cordilleran continental margin arc, eastern Transverse Ranges, California. In: Miller, R. B. \& Snoke, A.W. (eds) Crustal Cross Sections from the Western North American Cordillera and Elsewhere: Implications for Tectonic and Petrologic Processes. Geological Society of America, Special Papers 456, 187-218.

Nui, Y., Gilmore, T., Mackie, S., Greig, A. \& Bach, W. (2002). Mineral chemistry, whole-rock compositions, and petrogenesis of Leg 176 gabbros: data and discussion. In: Natland, J.H., Dick, H.J.B., Miller, D.J. \& Von Herzen, R.P. (eds) Proc. ODP, Sci. Results, 176, 1-60.

Otamendi, J. E., Tibaldi, A. M., Vujovich, G. I. \& Viñao, G. A. (2008). Metamorphic evolution of migmatites from the deep Famatinian arc crust exposed in Sierras Valle Fértil-La Huerta, San Juan, Argentina. Journal of South American Earth Sciences 25, 313-335.

Otamendi, J. E., Ducea, M., Tibaldi, A. M., Bergantz, G. W., de la Rosa, J. D. \& Vujovich, G. I. (2009a). Generation of tonalitic and dioritic magmas by coupled partial melting of gabbroic and metasedimentary rocks within the deep crust of the Famatinian magmatic arc, Argentina. Journal of Petrology 50, 841-873.

Otamendi, J. E., Vujovich, G. I., de la Rosa, J. D., Tibaldi, A. M., Castro, A., Martino, R. D. \& Pinotti, L. P. (2009b). Geology and petrology of a deep crustal zone from the Famatinian paleoarc, Sierras de Valle Fertil and La Huerta, San Juan, Argentina. Journal of South American Earth Sciences 27, 258-279.
Otamendi, J. E., Cristofolini, E., Tibaldi, A. M., Quevedo, F. I. \& Baliani, I. (2010a). Petrology of mafic and ultramafic layered rocks from the Jaboncillo Valley, Sierra de Valle Fértil, Argentina: Implications for the evolution of magmas in the lower crust of the Famatinian arc. Journal of South American Earth Sciences 29, 685-704.

Otamendi, J. E., Pinotti, L. P., Basei, M. A. S. \& Tibaldi, A. M. (2010b). Evaluation of petrogenetic models for intermediate and silicic plutonic rocks from the Sierra de Valle Fértil-La Huerta, Argentina: Petrologic constraints on the origin of igneous rocks in the Ordovician Famatinian-Puna paleoarc. Journal of South American Earth Sciences 30, 29-45.

Otamendi, J. E., Ducea, M. N. \& Bergantz, G. W. (2012). Geological, petrological and geochemical evidence for progressive construction of an arc crustal section, Sierra de Valle Fértil, Famatinian Arc, Argentina. Journal of Petrology 53, 761-800.

Pankhurst, R. J., Rapela, C. W., Saavedra, J., Baldo, E., Dalhlquist, J., Pascua, I. \& Fanning, C. M. (1998). The Famatinian magmatic arc in the central Sierras Pampeanas: an Early to Mid-Ordovician continental arc on the Gondwana margin. In: Pankhurst, R. J. \& Rapela, C. W. (eds) The Proto-Andean Margin of Gondwana. Geological Society, London, Special Publications 142, 343-367.

Petterson, M. G., Crawford, M. B. \& Windley, B. F. (1993). Petrogenetic implications of neodymium isotope data from the Kohistan batholith, North Pakistan. Journal of the Geological Society, London 150, 125-129.

Pichavant, M. \& Macdonald, R. (2007). Crystallization of primitive basaltic magmas at crustal pressures and genesis of the calc-alkaline igneous suite: experimental evidence from St Vincent, Lesser Antilles arc. Contributions to Mineralogy and Petrology 154, 535-558.

Putirka, K. D., Canchola, J., Rash, J., Smith, O., Torrez, G., Paterson, S. R. \& Ducea, M. N. (2014). Pluton assembly and the genesis of granitic magmas: Insights from the GIC pluton in cross section, Sierra Nevada Batholith, California. American Mineralogist 99, 1284-1303.

Rapela, C. W., Pankhurst, R. J., Casquet, C., Baldo, E., Saavedra, J. \& Galindo, C. (1998). Early evolution of the Proto-Andean margin of South America. Geology 26, 707-710.

Rapp, R. P. (1991). Origin of Archean granitoids and continental evolution. EOS Transactions, American Geophysical Union 72, 225, 229.

Rapp, R. P. \& Watson, E. B. (1995). Dehydration melting of metabasalt at 8-32 kbar: implications for continental growth and crust-mantle recycling. Journal of Petrology 36, 891-931.

Ratajeski, K., Sisson, T. \& Glazner, A. (2005). Experimental and geochemical evidence for derivation of the El Capitan Granite, California, by partial melting of hydrous gabbroic lower crust. Contributions to Mineralogy and Petrology 149, 713-734.

Reiners, P. W., Nelson, B. K. \& Ghiorso, M. S. (1995). Assimilation of felsic crust by basaltic magma: Thermal limits and extents of crustal contamination of mantle-derived magmas. Geology 23, 563-566.

Rioux, M., Hacker, B., Mattinson, J., Kelemen, P., Blusztajn, J. \& Gehrels, G. (2007). Magmatic development of an intra-oceanic arc: High-precision $\mathrm{U}-\mathrm{Pb}$ zircon and whole-rock isotopic analyses from the accreted Talkeetna arc, south-central Alaska. Geological Society of America Bulletin 119, 1168-1184.

Rodriguez-Vargas, A., Koester, E., Mallmann, G., Conceição, R. V., Kawashita, K. \& Weber, M. B. I. (2005). Mantle diversity beneath the Colombian Andes, Northern Volcanic Zone: Constraints from $\mathrm{Sr}$ and $\mathrm{Nd}$ isotopes. Lithos 82, 471-484. 
Roeder, P. L. \& Emslie, R. F. (1970). Olivine-liquid equilibrium. Contributions to Mineralogy and Petrology 29, 275-289.

Ross, D. C. (1985). Mafic gneissic complex (batholithic root) in the southernmost Sierra Nevada, California. Geology 13, 288-291.

Saito, S., Arima, M., Nakajima, T. \& Kimura, J.-I. (2004). Petrogenesis of Ashigawa and Tonogi granitic intrusions, southern part of the Miocene Kofu Granitic Complex, central Japan: M-type granite in the Izu arc collision zone. Journal of Mineralogical and Petrological Sciences 99, 104-117.

Saito, S., Arima, M., Nakajima, T., Misawa, K. \& Kimura, J.-I. (2007). Formation of distinct granitic magma batches by partial melting of hybrid lower crust in the Izu arc collision zone, central Japan. Journal of Petrology 48, 1761-1791.

Samson, S. D., Patchett, P. J., Gehrels, G. E. \& Anderson, R. G. (1990). Nd and Sr isotopic characterization of the Wrangellia Terrane and implications for crustal growth of the Canadian Cordillera. Journal of Geology 98, 749-762.

Sisson, T. W. \& Grove, T. L. (1993). Experimental investigations of the role of $\mathrm{H}_{2} \mathrm{O}$ in calc-alkaline differentiation and subduction zone magmatism. Contributions to Mineralogy and Petrology 113, 143-166.

Sisson, T. W., Grove, T. L. \& Coleman, D. S. (1996). Hornblende gabbro sill complex at Onion Valley, California, and a mixing origin for the Sierra Nevada batholith. Contributions to Mineralogy and Petrology 126, 81-108.

Sisson, T. W., Ratajeski, K., Hankins, W. B. \& Glazner, A. F. (2005). Voluminous granitic magmas from common basaltic sources. Contributions to Mineralogy and Petrology 148, 635-661.

Smith, D. J. (2014). Clinopyroxene precursors to amphibole sponge in arc crust. Nature Communications 5 doi:10.1038/ ncomms5329.

Smith, I. E. M., Worthington, T. J., Price, R. C., Stewart, R. B. \& Maas, R. (2006). Petrogenesis of dacite in an oceanic subduction environment: Raoul Island, Kermadec arc. Journal of Volcanology and Geothermal Research 156, 252-265.

Spandler, C., Arculus, R., Eggins, S., Mavrogenes, J., Price, R. \& Reay, A. (2003). Petrogenesis of the Greenhills Complex, Southland, New Zealand: magmatic differentiation and cumulate formation at the roots of a Permian island-arc volcano. Contributions to Mineralogy and Petrology 144, 703-721.

Stern, C. R. (1991). Role of subduction erosion in the generation of Andean magmas. Geology 19, 78-81.
Sun, S. \& McDonough, W. F. (1989). Chemical and isotopic systematics of oceanic basalts: implications for mantle composition and processes. In: Saunders, A. D. \& Norry, M. J. (eds) Magmatism in the Ocean Basins. Geological Society, London, Special Publications 42, 313-345.

Tepper, J. H., Nelson, B. K., Bergantz, G. W. \& Irving, A. J. (1993). Petrology of the Chilliwack Batholith, North Cascades, Washington: Generation of calc-alkaline granitoids by melting of mafic lower crust with variable water fugacity. Contributions to Mineralogy and Petrology 113, 333-351.

Thomas, W. A. \& Astini, R. A. (1996). The Argentine Precordillera: a traveller from the Ouachita embayment of North American Laurentia. Science 273, 752-757.

Tibaldi, A. M., Otamendi, J. E., Cristofolini, E. A., Baliani, I., Walker, B. A. \& Bergantz, G. W. (2013). Reconstruction of the Early Ordovician Famatinian arc through thermobarometry in lower and middle crustal exposures, Sierra de Valle Fertil, Argentina. Tectonophysics 589, 151-166.

Vernon, R. H., Paterson, S. R. \& Geary, E. E. (1989). Evidence for syntectonic intrusion of plutons in the Bear Mountains fault zone, California. Geology 17, 723-726.

Vujovich, G., Godeas, M., Marín, G. \& Pezzutti, N. (1996). El complejo magmático de la Sierra de La Huerta, provincia de San Juan. Actas XIII Congreso Geológico Argentino y III Congreso de Exploración de Hidrocarburos 3, 465-475.

Walker, B. A. J., Miller, C. F., Lowery Claiborne, L., Wooden, J. L. \& Miller, J. S. (2007). Geology and geochronology of the Spirit Mountain batholith, southern Nevada: Implications for timescales and physical processes of batholith construction. Journal of Volcanology and Geothermal Research 167, 239-262.

Westerman, D. S., Dini, A., Innocenti, F. \& Rocchi, S. (2004). Rise and fall of a nested Christmas-tree laccolith complex, Elba Island, Italy. In: Breitkreuz, C. \& Petford, N. (eds) Physical Geology of High-Level Magmatic Systems. Geological Society, London, Special Publications 234, 195-213.

Wolf, M. B. \& Wyllie, P. J. (1994). Dehydration-melting of amphibolite at 10 kbar: the effects of temperature and time. Contributions to Mineralogy and Petrology 115, 369-383.

Yuan, X., Sobolev, S. V. \& Kind, R. (2002). Moho topography in the central Andes and its geodynamic implications. Earth and Planetary Science Letters 199, 389-402.

Zavala, K., Leitch, A. M. \& Fisher, G. W. (2011). Silicic segregations of the Ferrar Dolerite Sills, Antarctica. Journal of Petrology52, 1927-1964. 